\title{
Structural development of hippocampal CA3 pyramidal neurons and relation to the development of spatial learning and memory in rats
}

\author{
By
}

Maha Mahdi

A thesis submitted to the Faculty of Graduate and Postdoctoral Affairs in partial fulfillment of the requirements for the degree of

Master of Science

in

Neuroscience

\author{
Carleton University \\ Ottawa, Ontario \\ (C) 2015
}

Maha Mahdi 


\begin{abstract}
The dentate gyrus-CA3 circuit in the hippocampus is highly plastic during development and morphologically responsive to various environmental outputs. The current study investigated the structural changes in CA3 pyramidal neurons during development (p18-p24) and the effect of AMPA receptor antagonism on spatial learning and memory. Experiment 1 showed individual changes in the elements of CA3 pyramidal neurons. Some parts expanded with age and others retracted. Golgi analysis revealed a reduction in spine density and an increase in thorny excrescences (TE), suggesting a role for TE in spatial abilities that emerge at the same age. Experiment 2 investigated the effect of AMPAr antagonism (NBQX) on spatial function and on CA3 pyramidal neuron structure. NBQX-injected rats showed impaired spatial abilities and individual remodeling in different components of CA3 pyramidal neurons including an increase in spines density and a decrease in TE. This suggest a role for TE in mediating spatial learning and memory.
\end{abstract}




\section{Acknowledgments}

I would like to express my sincere gratitude to my supervisor Dr. Matthew Holahan for his patience, guidance and expertise throughout the duration of this project. I would also like to thank Catherine Smith and Shaina Cahill for guidance in the lab. 


\section{Table of Contents}

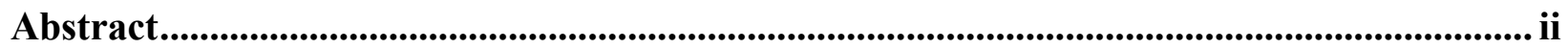

Acknowledgments ...........................................................................................................................

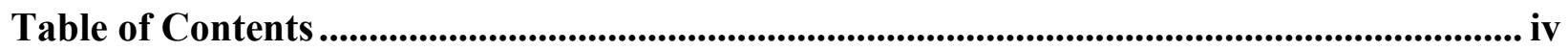

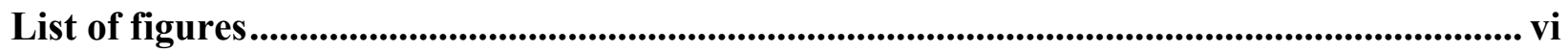

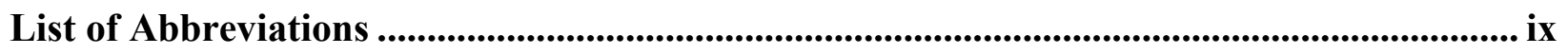

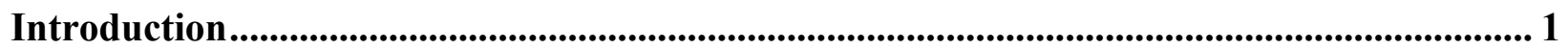

Spatial learning and memory in rodents................................................................ 1

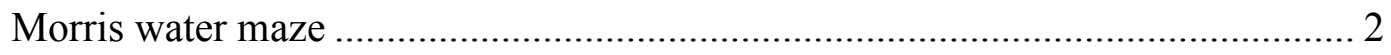

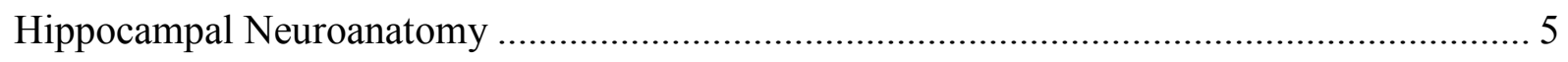

Thorny excrescences of CA3 pyramidal neurons ..................................... 8

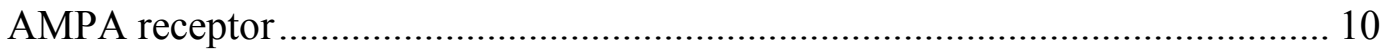

Hippocampal lesions and outcome on spatial learning and memory ................................. 11

Hippocampus lesions and spatial function................................................ 11

Hippocampal subregions lesions and spatial function ................................... 13

Morphological modifications in the hippocampus following spatial learning ..................... 15

DG-CA3 circuit morphological changes after spatial training in adult rats ........ 16

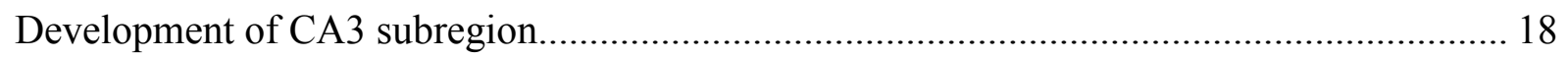

Overview of the Golgi-Cox technique ........................................................................ 21

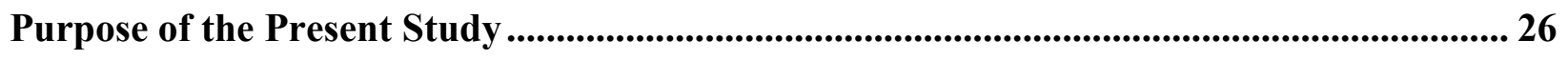

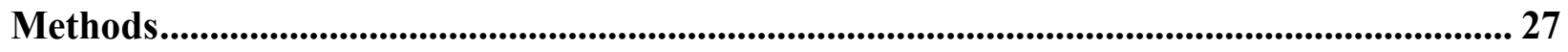

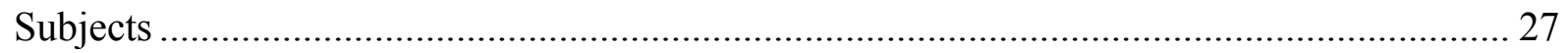

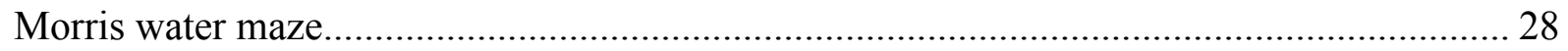




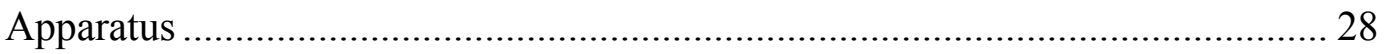

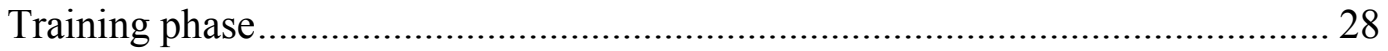

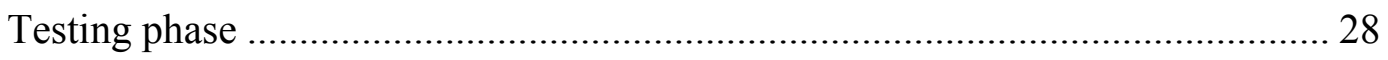

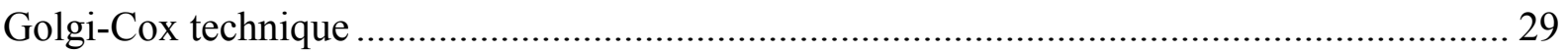

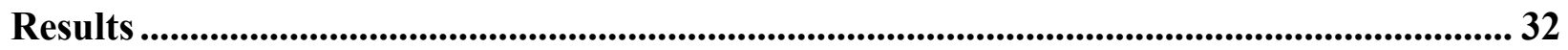

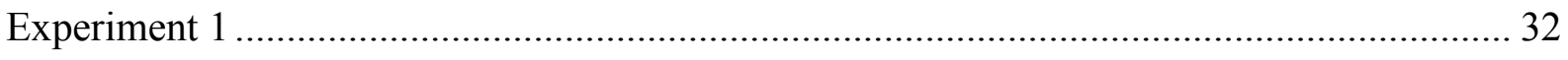

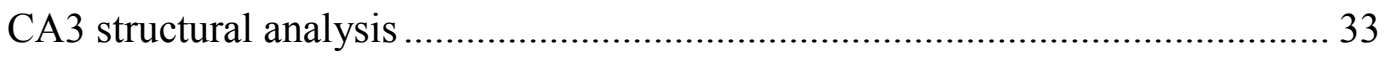

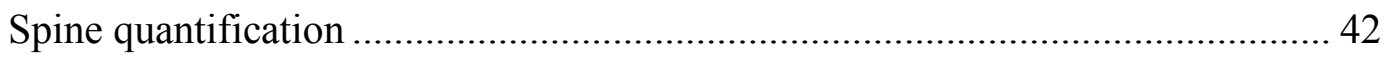

Thorny excrescence quantification ......................................................... 51

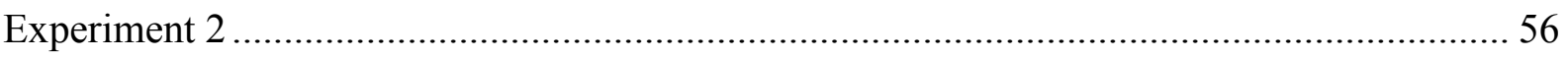

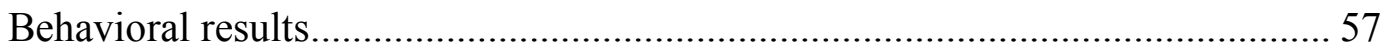

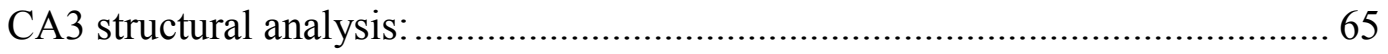

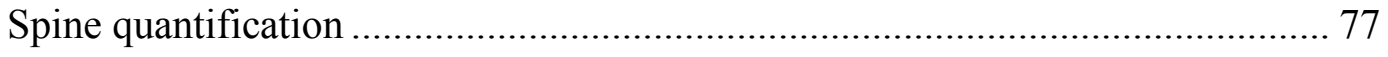

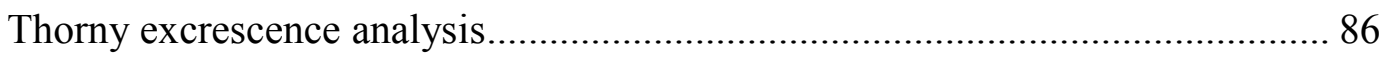

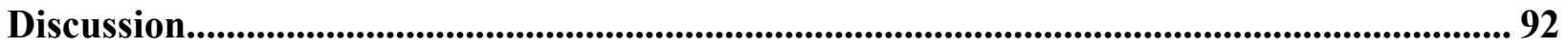

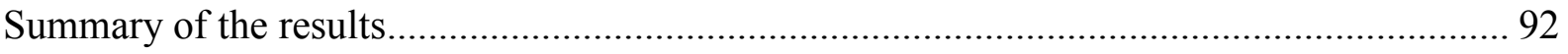

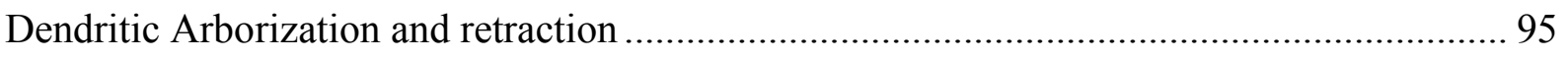

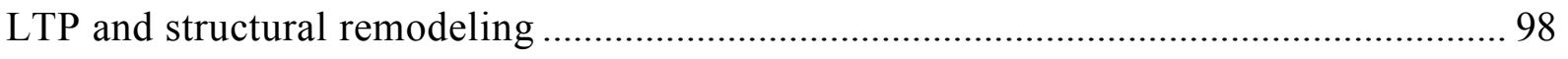

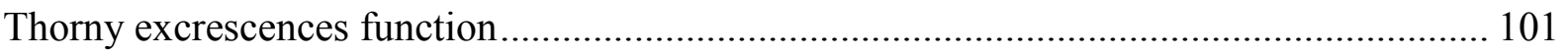

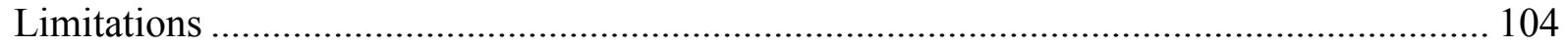

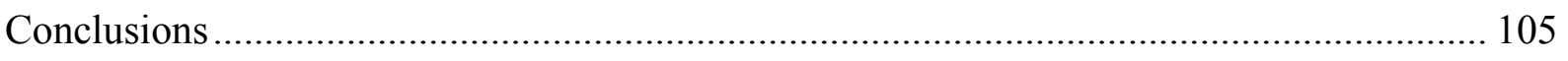

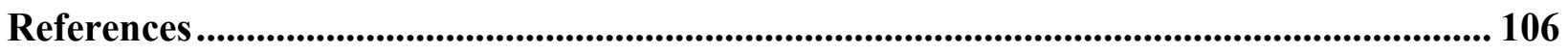




\section{List of figures}

Figure 1. Representative images for Golgi impregnation of the hippocampus ..................... 6

Figure 2. CA3 pyramidal neurons (Development groups): Representative images for Golgi impregnation of CA3 pyramidal neurons. .............................................................. 34

Figure 3. CA3 pyramidal neurons (Development groups): Area of the cell body.................... 35

Figure 4. CA3 pyramidal neurons (Development groups): Number of basal dendrites ............. 36

Figure 5. CA3 pyramidal neurons (Development groups): Number of apical dendrites............ 37

Figure 6. CA3 pyramidal neurons (Development groups): Quantification of nodes .................. 38

Figure 7. CA3 pyramidal neurons (Development groups): Quantification of ends................... 39

Figure 8. CA3 pyramidal neurons (Development groups): Total length ................................. 40

Figure 9. CA3 pyramidal neurons (Development groups): Apical and basal dendrites lengths.. 41

Figure 10. CA3 pyramidal neurons (Development groups): Surface and Volume..................... 42

Figure 11. CA3 pyramidal neurons (Development groups): Complexity................................ 43

Figure 12. CA3 pyramidal neurons (Development groups): Total number of spines ............... 44

Figure 13. CA3 pyramidal neurons (Development groups): Total spine density/ $\mu \mathrm{m} . . . \ldots \ldots \ldots \ldots . . . .45$

Figure 14. CA3 pyramidal neurons (Development groups): Total spine density/ dendrite ........ 46

Figure 15. CA3 pyramidal neurons (Development groups): Number of apical spines.............. 47

Figure 16. CA3 pyramidal neurons (Development groups): Apical spine density/ $\mu$ m............. 48

Figure 17. CA3 pyramidal neurons (Development groups): Spine density/ apical dendrite ....... 49

Figure 18. CA3 pyramidal neurons (Development groups): Number of basal spines ............... 50

Figure 19. CA3 pyramidal neurons (Development groups): Basal spine density/ $\mu \mathrm{m}$.............. 51

Figure 20. CA3 pyramidal neurons (Development groups): Basal spine density/ dendrite ...... 33 
Figure 21. CA3 pyramidal neurons (Development groups): Representative images for Golgi impregnation of TE on CA3 pyramidal neurons ........................................................ 53

Figure 22. CA3 pyramidal neurons (Development groups): Total number of TE.................... 54

Figure 23. CA3 pyramidal neurons (Development groups): Number of apical TE................... 55

Figure 24. CA3 pyramidal neurons (Development groups): Number of basal TE ................... 56

Figure 25. CA3 pyramidal neurons (Development groups): Number of TE on cell body .......... 52

Figure 26. Behavioral analysis (NBQX groups): Latency to reach the hidden platform......... 59

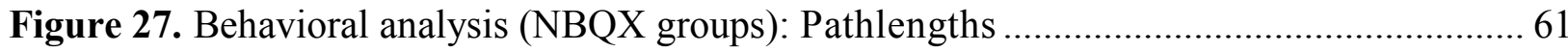

Figure 28. Behavioral analysis (NBQX groups): Thigmotaxis......................................... 63

Figure 29. Behavioral analysis (NBQX groups): Probe test results ................................... 65

Figure 30. CA3 pyramidal neurons (NBQX groups): Representative images for Golgi

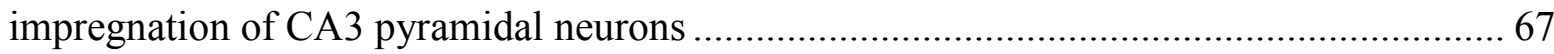

Figure 31. CA3 pyramidal neurons (NBQX groups): Areas of the cell bodies ....................... 68

Figure 32. CA3 pyramidal neurons (NBQX groups): Quantification of basal dendrites ........... 69

Figure 33. CA3 pyramidal neurons (NBQX groups): Quantification of apical dendrites .......... 70

Figure 34. CA3 pyramidal neurons (NBQX groups): Quantification of nodes ........................ 71

Figure 35. CA3 pyramidal neurons (NBQX groups): Quantification of ends.......................... 72

Figure 36. CA3 pyramidal neurons (NBQX groups): Total length ....................................... 73

Figure 37. CA3 pyramidal neurons (NBQX groups): Length of apical dendrites................ 74

Figure 38. CA3 pyramidal neurons (NBQX groups): Length of basal dendrites ..................... 75

Figure 39. CA3 pyramidal neurons (NBQX groups): Surface........................................... 76

Figure 40. CA3 pyramidal neurons (NBQX groups): Volume.......................................... 77

Figure 41. CA3 pyramidal neurons (NBQX groups): Complexity................................... 78 
Figure 42. CA3 pyramidal neurons (NBQX groups): Quantification of total spines............ 79

Figure 43. CA3 pyramidal neurons (NBQX groups): Total spine density per $\mu \mathrm{m}$................... 80

Figure 44. CA3 pyramidal neurons (NBQX groups): Total spine density per dendrite ............. 81

Figure 45. CA3 pyramidal neurons (NBQX groups): Quantification of apical spines............... 82

Figure 46. CA3 pyramidal neurons (NBQX groups): Apical spine density per $\mu \mathrm{m} . . . \ldots \ldots \ldots \ldots \ldots . . . . . .83$

Figure 47. CA3 pyramidal neurons (NBQX groups): Spine density per apical dendrite ........... 84

Figure 48. CA3 pyramidal neurons (NBQX groups): Quantification of basal spines ............... 85

Figure 49. CA3 pyramidal neurons (NBQX groups): Basal spine density per $\mu \mathrm{m}$.............. 86

Figure 50. CA3 pyramidal neurons (NBQX groups): Spine density per basal dendrite............. 66

Figure 51. CA3 pyramidal neurons (NBQX groups): Representative images for Golgi

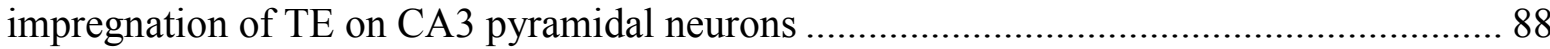

Figure 52. CA3 pyramidal neurons (NBQX groups): Number of total TE ............................. 89

Figure 53. CA3 pyramidal neurons (NBQX groups): Number of apical TE ............................ 90

Figure 54. CA3 pyramidal neurons (NBQX groups): Number of basal TE ........................... 91

Figure 55. CA3 pyramidal neurons (NBQX groups): Number of TE on cell bodies ................ 87 


\section{List of Abbreviations}

AMPA-a-amino-3-hydroxy-5-methyl-4-isoxazole propionic acid

ANOVA-analysis of variance

CA-Cornu Ammonis

CCAC-Canadian Council on Animal Care

DDC-diethyldithiocarbamate

DEHP-di(2-ethylhexyl) phthalate

DG-dentate gyrus

$\mathrm{DH}_{2} \mathrm{O}$-distilled water

dSO-dorsal stratum oriens

Dzp-diazepam

E-embryonic day

ETOH- Ethanol

FASD-Fetal Alcohol Spectrum Disorders

GluRs-Glutamate receptors

$\mathrm{HgCl}_{2}$-Mercuric Chloride

I.P-intraperitoneal injection

$\mathrm{K}_{2} \mathrm{Cr}_{2} \mathrm{O}_{4}$-Potassium Chromate 
$\mathrm{K}_{2} \mathrm{Cr}_{2} \mathrm{O}_{7}$-Potassium Dichromate

LER-Long Evans rats

LTP-long-term potentiation

mPFC-medial prefrontal cortex

MWM-Morris water maze

NBQX-2,3-dihydroxy-6-nitro-7-sulfamoyl-benzo[f]quinoxaline-2,3-dione

NMDA-N-methyl-D-aspartate receptors

OFC-orbitofrontal cortex

PBS-Phosphate-buffered saline

p-postnatal day

PSDs-postsynaptic densities

PTX-picrotoxin

SL-stratum lucidum

SO-Stratum oriens

SP-pyramidal cells layer

TE-Thorny excrescences

TTX-Tetrodotoxin

WR-Wistar rats 


\section{Introduction}

The investigation of the neural changes associated with learning and memory are a crucial matter of study in neuroscience. Several approaches have been developed to examine the changes in the nervous system that result from the interplay between organisms and their surroundings (Barnes, 1988). One of these approaches has been the study of learning and memory in rats whereby rats are trained on specially designed behavioural tasks that tap into spatial memory function and ensuing modifications regarding the dimensions, structure, or quantity of excitatory synapses in the hippocampus measured as potential sustained responses to spatial learning (Moser, Trommald \& Andersen, 1994).

The present study examined the morphological characteristics of hippocampal CA3 pyramidal cells during postnatal development in rats. Moreover, the study investigated the effect of administration of different doses of an AMPA receptor antagonist during this developmental period and the long-term effects on spatial learning and memory as well as changes in morphological characteristics of CA3 neurons. One over-arching and novel analysis included in both of these manipulations is to quantify and characterize thorny excrescences on CA3 pyramidal neurons during postnatal development and in rats that were injected with the AMPA receptor antagonist and assessed for their spatial function. The Golgi-based quantification and analysis of thorny excrescences in the CA3 region during development and after spatial learning has never been carried out so represents a novel procedural and analytical technique.

\section{Spatial learning and memory in rodents}

The neural bases of spatial function are often defined in terms of an ability to process the relationship between the locations of different objects in an environment in a three-dimensional 
coordinate system within the brain. Spatial learning occurs when an animal encodes details about their surroundings that are then used to navigate through space and remember places of motivational significance (Floresco, 2014).

Spatial learning is typically examined in terms of allocentric or egocentric cues, where allocentric cues are defined in reference to the environment and egocentric cues are in reference to oneself. Since the early twentieth century, many studies have investigated allocentric spatial learning and memory in rodents using various tasks such as T-mazes, radial arm mazes and water mazes. These studies have contributed to the general understanding of the neural basis of spatial learning and memory that may have applications to a number of other species, including humans.

Here, the focus lies on the Morris Water maze, one of the most widely used tools in the study of spatial learning in rodents. An overview of neurobiological data obtained from rats during their performance in this task showed that various brain systems contribute to spatial navigation. Specifically, spatial navigation involved the hippocampal formation and the associative parietal cortex (Poucet \& Benhamou, 1997).

\section{Morris water maze}

The Morris water maze has been used reliably to examine the ability of an animal to remember the location of a platform in a pool of water, using external cues for allocentric guidance and spatial navigation. The task was designed by Richard Morris to provide a robust and reliable estimate of spatial learning in rodents via use of allocentric cues (Morris, 1981; 1984). The task was developed as a result of theatrical debates about the neurological underpinnings of spatial and working memory (O'Keefe and Nadel, 1978; Olton \& Paras, 1979) 
and from the hypothesis that the basis of learning spatial associations might be distinct from other types of associative learning (Morris, 1984).

Performance in the water maze task is influenced by a combination of the intrinsic characteristics of the animals being tested (including strain and gender) and by differences in apparatus and training procedures. A general review of factors such as age, strain and gender differences as well as a complete description of the application and procedure involved in water maze testing has been described in D'Hooge \& De Deyn (2001). Moreover, evidence supports the use of the water maze as a measure for hippocampal-dependent spatial learning and memory (as cited in Barnes, 1988; McDonald, Hong \& Devan, 2004), and it provides a unique measurement of place learning (Vorhees \& Williams, 2006).

The apparatus consists of a relatively large circular container filled with water and a hidden platform. The top surface of the platform is placed under the surface of the water so as not to be visible to the rat and not provide a local cue to guide escape behavior. At first, a rat is put in the water to escape on the hidden platform. During this time, rats normally will swim at random or in unorganized exploration pathways. After a few trials, rats will swim more directly to the platform regardless of the starting location. The direction or heading, and reductions in pathlength and latency to the hidden platform are indications that the rat has learned the location of the platform in accordance with the distal cues (Morris, 1984).

The strength of the memory for the platform is examined by a testing trial that includes removal of the hidden platform and measuring the time the animal spends in the previous location of the platform. A number of dependent variables can be assessed during both the acquisition and testing phases including: The time required to find the platform (aka, latency), 
length of the swimming path, swimming speed, and during the probe test: time the animal spends swimming in each quadrant, time spent dwelling in the platform location and number of platform crossings (Sharma, Rakoczy \& Brown-Borg, 2010).

Various experimental strengths have led to the reliable and consistent use of the Morris water maze across a number of studies throughout the years (Vorhees \& Williams, 2006). Rats can swim naturally and so there is no need for extensive pretraining. In addition, there is no need to deprive the animals of food to motivate learning nor use electric shock to stimulate escape behavior (Morris, 1984).

Pups and adult rats use a variety of cues in order to navigate in a Morris water maze (MWM), including direction and distance cues (Akers \& Hamilton, 2007). Rats are able to swim by postnatal day $\mathrm{p} 16$, but require a mature hippocampus in order to accurately succeed in the MWM task during the probe test (Rossier \& Schenk, 2003). Rats can demonstrate adequate spatial navigation skills as early as p17 despite their relatively immature hippocampi (Carman \& Mactutus, 2001).

Vision plays a large role in spatial navigation in rats, whereas olfaction and audition play more minor roles (Rossier \& Schenk, 2003); however, if vision is impaired, audition and olfaction are more heavily relied on for spatial navigation. The principle of interocular equivalence dictates that neural networks from each eye must converge on neural structures mediating memory formation in order for visual cues to be properly integrated (Rudy \& Paylor, 1987). Rats do not demonstrate interocular equivalence before $\mathrm{p} 28$, thus may be unable to integrate visual cues until this age (Rossier \& Schenk, 2003). It is speculated that cues in multiple modalities may be important when training rats younger than $\mathrm{p} 28$. 
The Morris water maze task (MWM) has frequently been used to validate various rodent models of neurocognitive impairments (Radecki, Brown, Martinez \& Teyler, 2005) and to assess potential treatments (Gage \& Bjorklund, 1986). Because of its efficiency in illustrating spatial memory and navigation skills, the MWM and hippocampal function have become theoretically intertwined (D’Hooge \& De Deyn, 2001).

For example, rats were used to investigate if preadolescent exposure to water maze or a dry maze task could result in improvement in the performance on water maze and alternations in hippocampal connections (Wartman, et al., 2012). Results showed that experiencing water maze or a dry maze thorough the preadolescent period led to improved spatial performance on the water maze during adolescent period. This was correlated with rearrangements found in the axonal inputs received in CA3 area (Wartman, et al., 2012).

\section{Hippocampal Neuroanatomy}

The hippocampus is one of the evolutionary primitive regions of the brain. It is located in the temporal lobe and is part of the limbic system. The hippocampus is divided into two main regions; Cornu Ammonis (CA) and the dentate gyrus (see Figure 1a). The CA is structurally and functionally classified into distinctive subregions referred to as CA1, CA2, CA3, and CA4. The dentate gyrus consists of the granule cell layer with its dendrites and the hilus (Cherubini \& Miles, 2015). The dentate gyrus is a U-shaped single layer (a thickness of five to ten cells) that contains mainly granule cells that originate axons named mossy fibers. The CA1, CA2 and CA3 consist of large cell bodies of pyramidal neurons (Teyler \& DiScenna, 1984). 

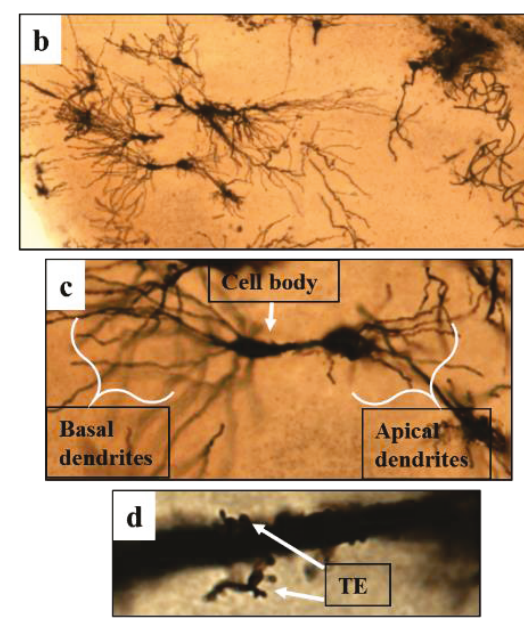

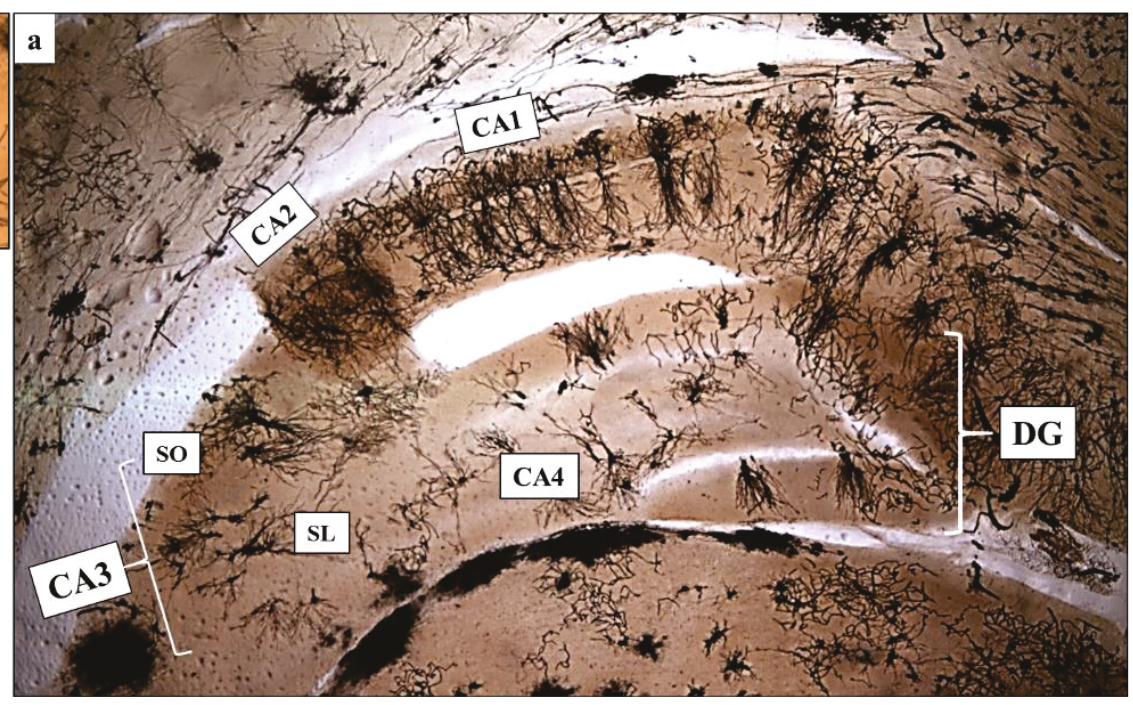

Figure 1. a. Representative images for Golgi impregnation of the hippocampus and its subregions: DG, CA1, CA2, CA3 (including SL and SO) and CA4 (2.5X). b. CA3 subregion (4X). c. A CA3 pyramidal neuron (20X). d. Thorny excrescences on a CA3 pyramidal neuron (100X).

The hippocampus is often identified as a tri-synaptic circuit in which information flows from the entorhinal cortex through the perforant path to the dentate gyrus, from dentate gyrus by mossy fibers to CA3 area, and from CA3 via the Schaffer collateral axons to CA1(Amaral and Witter, 1989; Teyler \& DiScenna, 1984). This circuit has been popularly described as a one-way pathway as the dentate gyrus does not project back to the entorhinal cortex (Teyler \& DiScenna, 1984).

Linked to this idea is evidence that the majority of information in the hippocampus is processed via this tri-synaptic circuit. Nevertheless, it is important to consider that the trisynaptic circuit might not be the only path for processing information and that the entorhinal cortex is not the only main external input to the hippocampus. Inputs from different sources are 
sent to the CA3 region as well, which makes it, besides the dentate gyrus, another entryway to the hippocampus (Scharfman, 2007).

As investigated by O'Keefe and Nadel (1978) recurrent collateral synapses within the CA3 subfield are more abundant than in other hippocampal regions. Repeated axon collaterals project extensively within the $\mathrm{CA} 3$ pyramidal cells creating excitatory connections along with nearby excitatory and inhibitory neurons (O'Keefe \& Nadel, 1978). In addition, axons of CA3 pyramidal cells project back to the granule cells in the dentate gyrus (Scharfman, 2007).

In 1971, it was proposed that the dentate gyrus receives excitatory inputs from entorhinal cortex in a topographical way, and via the "tri-synaptic circuit" spreading through horizontal "slices or "lamellae" of the hippocampus (Andersen, Bliss \& Skrede, 1971). Considering this idea, independent functions can be mediated by a comparatively small part in a way similar to the way separate piano keys create almost an endless number of possible distinctive outcomes. The lamellar theory gets essential backing from the "lamellar" appropriation of mossy fibers (dentate granule cell axons) that synapse on dentate hilar neurons and CA3 pyramidal cells and interneurons inside the limitations of a slim transverse hippocampal portion (Sloviter \& Lømo, 2012).

The lamellar hypothesis was reliable with the acknowledged neuroanatomy of the hippocampus. However, following neuroanatomical examinations using various new tracing methods, it has been shown that the majority of hippocampal axons, aside from mossy fibers, significantly diverge, which is counter to what the lamellar hypothesis implies (Amaral \& Witter,1989). 


\section{Thorny excrescences of CA3 pyramidal neurons}

The CA3 subregion includes two subfields; the stratum lucidum (SL) and the stratum oriens (SO) (See Figure 1a, b and c). The SL subfield is distal to the dentate gyrus and proximal to the CA3 pyramidal cell layer consisting of the apical dendrites (Vida \& Frotscher, 2000). The SL contains the majority of mossy fiber terminals that make synaptic connections on the apical dendrites of CA3 pyramidal neurons (Blackstad, 1956; Raisman, Cowan \& Powell, 1965; Vida \& Frotscher, 2000). The SO is composed of the basal dendrites of the CA3 pyramidal neurons and the Schaffer collaterals that project to the CA1 (Raisman, Cowan \& Powell, 1965; Vida \& Frotscher, 2000).

A unique type of dendritic spine called thorny excrescence is found in the CA3 pyramidal neurons (see Figure 1d). Thorny excrescences are one of the most morphologically distinctive synapses in the mammalian brain. They are large spines that show complex shapes (Gonzales, DeLeon Galvan, Rangel \& Claiborne, 2001; Amaral, 1978; Stirling \& Bliss, 1978; Fitch, Juraska \& Washington, 1989). Thorny excrescences make up the postsynaptic targets of the mossy fiber terminals of granule cells from the dentate gyrus. Ultrastructural examinations show that a thorny excrescence is made up of a slender neck joining 1 to 16 bulbous-shaped heads to the dendritic shaft (Blackstad \& Kjaerheim, 1961; Amaral \& Dent, 1981; Chicurel \& Harris, 1992). Mostly, one excrescence is connected with a single mossy fiber bouton, yet a mossy bouton can make contacts with multiple excrescences (Chicurel \& Harris, 1992; Acsa'dy, Kamondi, S1'k , Freund \& Buzsa'ki, 1998). According to one study, "a pyramidal neuron can be contacted by a maximum of 41 mossy fiber boutons, each from a different granule cell "(Gonzales, DeLeon Galvan, Rangel, \& Claiborne, 2001). 
The density, extent and spread of excrescence clusters in CA3 pyramidal neurons in adult rats has been previously measured using three-dimensional evaluation method along with light microscopy (Gonzales, DeLeon Galvan, Rangel, \& Claiborne, 2001). Neurons of the midfield of CA3 as well as neurons located proximal to the hilus of the dentate gyrus were analysed. Results revealed that proximal CA3 neurons, located closer to the hilus of dentate gyrus, have a similar density of clusters on their apical and basal dendrites and these clusters show similar lengths (Gonzales, DeLeon Galvan, Rangel, \& Claiborne, 2001). On the other hand, midfield neurons, located within the midportion of CA3 subregion, have a greater number of clusters on apical dendrites compared to basal dendrites, and apical clusters were also longer. Basal clusters in neurons within both areas (midfield and proximal) were positioned around $50 \%$ more adjacent to the cell body. In general, proximal neurons have a greater number of clusters than neurons in the midfield. However, clusters of midfield neurons were longer; hence, the total lengths of clusters in both areas were similar and seemingly contained a similar amount of single excrescences (Gonzales, DeLeon Galvan, Rangel, \& Claiborne, 2001).

It is proposed here that the clusters of thorny excrescences help to convey information from the presynaptic mossy fibers that exhibit remodeling following spatial learning (Holahan, Rekart, Sandoval \& Routtenberg, 2006; McGonigal, Tabatadze \& Routtenberg, 2012; RamírezAmaya, Balderas, Sandoval, Escobar \& Bermúdez-Rattoni, 2001; Rekart, Sandoval, BermudezRattoni \& Routtenberg, 2007) especially in the excitatory terminals that make contact with the CA3 pyramidal neurons (McGonigal, Tabatadze \& Routtenberg, 2012). Thus presynaptic component of DG-CA3 thorny excrescences may enhance spatial processing on a variety of spatial tasks such as the MWM task. In addition, it is speculated that juvenile rats at p24 would exhibit more and larger thorny excrescences than that of rats in p18, p20, and p22 as it is the time 
that rats reach maturity in spatial learning and memory (Rudy, Stadler-Morris \& Albert, 1987;

Keeley, Wartman, Hausler \& Holahan, 2010).

\section{AMPA receptor}

Glutamate and its receptors (GluRs) mediate the excitatory synaptic transmission in the DG-CA3 circuit and underlie synaptic plasticity (Cotman, Monaghan \& Ganong, 1988; Wisden \& Seeburg, 1993; Shi, et al., 1999). Glutamate acts on two different receptor types: ionotropic and metabotropic (Nakanishi \& Masu, 1994; Salt \& Eaton, 1996). Ionotropic glutamate receptors of mammals are divided based on their pharmacological and molecular elements into the aamino-3-hydroxy-5-methyl-4-isoxazole propionic acid (AMPA), kainate, and N-methyl-Daspartate (NMDA) receptors (Hollmann \& Heinemann, 1994).

In Experiment 2 in the current study, AMPA receptors were blocked with NBQX (2,3dihydroxy-6-nitro-7-sulfamoyl-benzo[f]quinoxaline-2,3-dione). It is proposed here that blocking AMPA receptors prevents DG-CA3 circuit excitatory transmission, normal plasticity and may impair spatial learning and memory. An AMPAr antagonist but not an NMDAr antagonist was used in this study because, although blocking NMDAr has been shown to impair spatial abilities, it also results in locomotor deficits i.e. animals cannot move or swim in the MWM (Ahlander, Misane, Schott, \& Ogren, 1999; unpublished observations).

AMPA is the most abundant receptor in the vertebrate nervous system and mediates rapid synaptic transmission. They were first referred to as quisqualate receptors (Davies, Evans, Jones, Smith \& Watkins, 1982) as they were named after the natural agonist quisqualate. Later, they were given the name AMPA receptors after the artificial agonist (Honoré, Lauridsen \& Krogsgaard-Larsen, 1982). 
AMPA receptors are made of different chemical compositions formed by four similarly structured pore-forming subunits (GLuR1-4) that make various combinations of AMPA receptor types. (GLuR1-4) subunits are believed to possess "a large extracellular amino-terminal domain, three transmembrane domains and an intracellular carboxy-terminal domain" (Dong, et al., 1997). AMPA receptors are permeable to the influx of sodium into the cell (Malenka, 1994). It has been found that AMPA receptors concentrate heavily on the pyramidal neurons of the hippocampal CA1 and CA3 regions (Hampson, et al., 1992). It has been suggested that AMPA receptors are essential for learning and memory as they have such a high concentration in the hippocampus (Pandey, Rai, Gaur \& Prasad, 2015; Xie, et al., 2015; Mao, Xue, Jin \& Wang, 2015; El Iskandrani, Oosterhof, El Mansari \& Blier 2015).

\section{Hippocampal lesions and outcome on spatial learning and memory}

\section{Hippocampus lesions and spatial function}

A variety of manipulations to the hippocampus have been applied to examine its contribution to spatial memory including selective lesions of the entire hippocampal formation or particular subregions (Dentate gyrus, CA1 and CA3) (Barnes, 1988). In general, results have revealed that the performance of rats without a fully functioning hippocampus was significantly impaired on spatial learning and memory tasks but performance on non-spatial tasks is preserved (Jarrard, 1993).

One study compared the effect of hippocampal lesions and lesions of the fimbria-fornix on the acquisition and retention of spatial information (Cassel, et al., 1998). The fimbria-fornix is an intensively myelinated bundle of axons (mainly efferent) that rises from the subiculum and CA1 region of the hippocampus and projects to the anterior nuclei of the thalamus via the 
mammillary bodies. Using both the Morris water maze and eight-arm radial maze tasks, behavioral results showed that performance of rats with hippocampus lesions was greatly impaired compared to the performance of rats that had fimbria-fornix lesions (Cassel, et al., 1998).

In another study, rats received ibotenic acid-induced lesions of the hippocampus and/or the subiculum (a subcortical structure located between the hippocampus proper and entorhinal cortex) and were then assessed for their spatial learning and memory function via training and testing on the water maze task. Behavioral results revealed that rats with lesions of both the hippocampus and subiculum were impaired in initial acquisition of the task and when those rats were extensively trained, they showed deficits in place learning (Morris, Schenk, Tweedie \& Jarrard, 1990).

In the same study, when comparing a hippocampus- only lesioned group with a subiculum-only lesioned group, results showed that both groups used different strategies in searching for the hidden platform during training phase (Morris, Schenk, Tweedie \& Jarrard, 1990). While hippocampus-only lesioned rats exhibited stereotyped strategy swimming in circles around all of the pool or a part of it, the subiculum-only lesioned rats displayed patterns of searching that were similar to those of normal rats that did not know the location of the platform. When they were tested in a delayed matching to place task, both groups demonstrated impairments, but the subiculum-lesioned group performed better than the hippocampus-lesioned group (Morris, Schenk, Tweedie \& Jarrard, 1990). Result revealed that hippocampal lesions given after training resulted in retention and relearning deficits compared to a sham group (Morris, Schenk, Tweedie \& Jarrard, 1990). 
In another study, complete cytotoxic hippocampal lesions were performed in rats to examine the impact on spatial learning and memory. Behavioral results revealed that rats without a hippocampus displayed spatial deficits in both the water-maze and spatial T-maze tasks (Bannerman, et al., 1999).

\section{Hippocampal subregions lesions and spatial function}

Many studies have looked at the impact of lesions and inactivation of specific hippocampal subregions, in particular, the CA3 region, on spatial learning and memory. Various phases of learning and memory were examined using spatial and non-spatial versions of the water maze task after lesions or inactivation of the dorsal hippocampal CA3 subregion (Florian $\&$ Roullet, 2004). Mice were focally injected with diethyldithiocarbamate (DDC) to inactivate the CA3 subregion temporarily (Florian \& Roullet, 2004). DDC can reversibly chelate heavy metals, such as zinc, that are prominent in the synapses between the mossy fiber buttons and the CA3 pyramidal cells. When tested on the spatial version of MWM task, mice that received bilateral injections of DDC into the CA3 subregion were impaired in acquiring but not in remembering spatial information. In the non-spatial (cued) version of MWM, mice with CA3 subregion inactivation performed as controls in both acquiring and remembering non-spatial information (Florian \& Roullet, 2004). These conclusions suggest that the hippocampal CA3region is necessary for spatial memory processes particularly in memory consolidation of spatial information (Florian \& Roullet, 2004).

In another study, rats were trained on an object-cued spatial location recall task and a spatial location-cued object recall task after they were given either a dorsal CA3 lesion or control lesion. When tested on both tasks, CA3-lesioned rats showed impairments in both tasks 
compared to controls, but they performed as well as controls on a non-episodic version of the task (Kesner, Hunsaker \& Warthen, 2008).

Rats received lesions to either CA3 or CA1 after they were trained on an episodic memory version of water maze task. Results revealed that all lesioned rats had impaired episodic memory processing, but CA1-lesioned rats showed greater impairment (Hunsaker, Lee \& Kesner, 2008). Both CA1 and CA3 lesions had no impact on a non-episodic version of the MWM. These findings indicate that both CA3 and CA1 process spatial episodic information but not non-episodic information (Hunsaker, Lee \& Kesner, 2008).

In a related study, rats with either CA3 or CA1 lesions were trained on an object-traceplace paired-associate learning task. Both groups exhibited different impairments in the acquisition of this task. While CA3 lesioned rats were impaired in associating spatial details, CA1 lesioned rats were impaired in associating temporal (trace) details (Hunsaker, Thorup, Welch \& Kesner, 2006). These findings indicate that CA1 and CA3 interact when a task involves both spatial and temporal information (Hunsaker, Thorup, Welch \& Kesner, 2006).

Lidocaine-induced CA3 inactivation in rats and the associated outcome on spatial learning and memory has been studied. Rats were chronically implanted and bilaterally injected with Lidocaine, targeting the mossy fiber terminal field in the CA3 region every day after being trained on water maze task for five days. Rats were tested on a probe test seven days after the last day of training (Holahan \& Routtenberg, 2011). Results showed that the lidocaine-injected group performed worse than phosphate-buffered saline (PBS)-injected controls in learning and remembering the platform location in the first trial of each training day. During the probe test, the lidocaine-injected group was significantly impaired in remembering the location of the 
platform compared to controls. These data suggested that reduced activity in the CA3 region resulted in both short-term deficits and long-term spatial memory dysfunction (Holahan \& Routtenberg, 2011).

In one research, lesion to the dentate gyrus was induced by neonatal X-ray irradiation at 6, 18, or 24 hours following birth (Czéh, et al., 2001). Dentate gyrus lesioned rats were evaluated in adulthood on the Morris water maze task. Results showed that irradiated rats were significantly impaired (Czéh, et al., 2001).

\section{Morphological modifications in the hippocampus following spatial learning}

Morphological adjustments, such as those that occur to dendrites and axons, following spatial learning in subregions of hippocampus have been also investigated. The dendritic spine density in the CA1 region in rats trained on the water maze task was compared to non-trained control animals (Stewart, et al., 2005). The dendritic spine density was utilized as an indicator for the density of hippocampal excitatory synapses. Results showed that trained animals had a significantly increased spine density on basal dendrites of CA1 pyramidal cell, which was correlated with spatial learning (Moser, Trommald \& Andersen, 1994). Further structural analysis revealed that the number of spines on the basal but not apical dendrites increased following water maze training (Moser, Trommald, Egeland \& Andersen, 1997).

The effect of chronic stress and spatial training on the structure of CA3 thorny excrescences has been demonstrated in adult rats by obtaining three-dimensional (3D) electron microscopic images of reconstructions of these cells (Stewart, et al., 2005). Quantitative 3-D data revealed that thorny excrescences were reduced following chronic restraint stress and reversed 
following water maze training. When animals were trained on water maze alone in the absence of restraint stress, the size and numbers of thorny excrescences were increased. Restraint stress did not have an effect on the postsynaptic densities (PSDs) on the surface of thorny excrescences; however, training on water maze expanded the area and number of PSDs (Stewart, et al., 2005). In addition, following restraint stress, quantity of endosome-like structures in thorny excrescences reduced while it increased following training on water maze (Stewart, et al., 2005).

\section{Dentate gyrus-CA3 circuit morphological changes after spatial training in adult rats}

Within the context of the trisynaptic loop view of the hippocampus, the dentate gyrus forms the main input to the CA3 pyramidal neurons via the mossy fibers that comprise the presynaptic component with the thorny excrescence on CA3 pyramidal neurons. Various studies have looked at the morphological changes in the dentate gyrus-CA3 circuit following training on spatial tasks in adult rats (Holahan, Rekart, Sandoval \& Routtenberg, 2006; McGonigal, Tabatadze \& Routtenberg, 2012; Rekart, Sandoval, Bermudez-Rattoni \& Routtenberg, 2007; Ramírez-Amaya, Balderas, Sandoval, Escobar \& Bermúdez-Rattoni, 2001)

The impact of water maze spatial training on reorganization of the presynaptic mossy fiber terminal field from the stratum lucidum to the distal stratum oriens was studied in male Long Evans (LER) and Wistar (WR) rats (Holahan, Rekart, Sandoval \& Routtenberg, 2006). Before training, LER exhibited a widespread endogenous expansion of the mossy fiber terminal field innervating the distal stratum oriens than that exhibited by WR. LER also were better than WR in remembering the location of the hidden platform and in a visible reversal water maze task (Holahan, Rekart, Sandoval \& Routtenberg, 2006). Both strains showed a significant 
reorganization of the mossy fiber terminal field into the distal stratum oriens seven days after being trained on the water maze task. However, LER exhibited significant extension of mossy fiber terminal field 24 hours following training (Holahan, Rekart, Sandoval \& Routtenberg, 2006). These results indicated that remodeling of the mossy fiber terminals into the distal stratum oriens may form a neural basis for the formation of long-term memory and efficient spatial navigations skills (Holahan, Rekart, Sandoval \& Routtenberg, 2006).

The effect of training adult rats in either a hidden platform water maze task or a visible platform water maze task on mossy fiber terminal remodeling was studied (McGonigal, Tabatadze \& Routtenberg, 2012). Animals were trained in one of the tasks for five days then tested seven days later. Rats that were trained on the hidden platform water maze task were found to have a significant increase in the number of large but not small mossy fiber terminals in the proximal stratum lucidum and the stratum oriens compared to controls and to rats trained on the visible water maze task (McGonigal, Tabatadze \& Routtenberg, 2012). The difference found between large and small mossy fiber terminals is functionally crucial because whereas large terminals target CA3 pyramidal neurons, small terminals target inhibitory interneurons. This finding suggests that spatial learning facilitates exclusive remodeling of excitatory inputs to the CA3 pyramidal neurons (McGonigal, Tabatadze \& Routtenberg, 2012).

In another study, an increase of the mossy fiber terminal field was found seven days but not two days following training on the hidden platform water maze task (Rekart, Sandoval, Bermudez-Rattoni \& Routtenberg, 2007). Thus, the formation of spatial memory resulted in structural changes of the mossy fiber-CA3 circuit that may be contribute to the maintenance of long-lasting spatial representations (Rekart, Sandoval, Bermudez-Rattoni \& Routtenberg, 2007). 
A study examined whether or not the water maze overtraining- induced hippocampal mossy fiber synaptogenesis can also be associated with long-lasting spatial memory (RamírezAmaya, Balderas, Sandoval, Escobar \& Bermúdez-Rattoni, 2001). Rats were trained for from one to five days and tested on the water maze twice: a week and a month after training. Results demonstrated that rats performed better with more training (five days better than one) in both probe tests (a week and a moth after training). This improvement was positively correlated with increased synaptogenesis in the stratum oriens especially in the septal region of the dorsal hippocampus supporting the idea that the septal region of the hippocampus is crucial for spatial memory (Ramírez-Amaya, Balderas, Sandoval, Escobar \& Bermúdez-Rattoni, 2001).

\section{Development of CA3 subregion}

The hippocampal formation develops rapidly during the embryonic period and early postnatal days in rats (Altman, 1969). The morphogenesis of the hippocampal formation in the rat was examined during embryonic and early postnatal periods (Altman, 1969; Bayer, 1979, 1980). A study of the morphogenesis of the entire hippocampal formation in normal rats revealed that the hippocampus becomes noticeable at embryonic day 16 (E16) (Bayer, 1979). The volume of hippocampal formation grows rapidly (by 1900\%) in embryonic days 16 and 17 . The hippocampus volume increases by $66 \%$ between E18 and E19, by $29 \%$ in E19 and E20, by $26 \%$ daily between $\mathrm{p} 1$ and $\mathrm{p} 7$, and by $12 \%$ daily between p7 to p21 (Bayer, 1980).

Mossy fibers, axons of granule cells in the dentate gyrus, show rapid axonal changes in the CA3 subregion during development (Holahan, Honegger, \& Routtenberg, 2007). The neural extension and retraction of mossy fibers in the CA3 region has been examined in Long-Evans rats using Timm's staining technique (Holahan, Honegger, \& Routtenberg, 2007). Timm's is a 
silver staining method used as a marker for zinc (Danscher \& Zimmer, 1978) which is found in high concentrations at the mossy fiber buttons (Frederickson, Klitenick, Manton, \& Kirkpatrick, 1983). Thus, a stained mossy fiber terminal is an indication of a presynaptic connection with thorny excrescences on a CA3 pyramidal neuron. Results revealed that at $\mathrm{p} 0$ to $\mathrm{p} 3$, rats exhibited a high quantity of CA3 pyramidal cells but no Timm's staining was detected in either SL or dorsal stratum oriens dSO areas of CA3 subregion (Holahan, Honegger, \& Routtenberg, 2007).

In the same study, between $\mathrm{p} 6$ and $\mathrm{p} 9$, a sign of Timm's stain was detected in the SL, and it was easily observable by $\mathrm{p} 12$ in the SL but not dSO. At p15, a dark band of Timm's stain was seen at the SL as well as scatters of Timm's staining in the CA3 pyramidal cells layer SP. Timm's staining appeared in SP and dorsal aspects of dSO by p18, and it was increased in the dSO at p21. An extensive staining in $\mathrm{dSO}$ was recognized at p24, and it was continued to $\mathrm{p} 33$ and into adulthood p40 (Holahan, Honegger, \& Routtenberg, 2007). These finding show that the expansion of the mossy fiber happened mainly during the development period between p18 and p24 implying morphological modifications that might help the rats to perform spatial task i.e. the MWM task.

The sensitive developmental period of the dentate gyrus-CA3 (from p16 to p24) in LER has been behaviorally tested (Rudy, Stadler-Morris \& Albert, 1987; Keeley, Wartman, Hausler \& Holahan, 2010). The development of spatial navigation behaviors using proximal and distal cues was examined in LER rats (Rudy, Stadler-Morris \& Albert, 1987). Rats use both proximal and distal cues to find or reach targets in their environment (Morris, 1981). A proximal cue is a bounded stimulus that coexists with the target, and it can guide behavior. On the other hand, a distal cue does not coexist with the target object; hence, to find the target, rats should learn about 
the spatial location of the target corresponding to distal cues (Rudy, Stadler-Morris \& Albert, 1987).

Juvenile rats were trained and tested using two versions of Morris water maze: a visible platform (a proximal cue) and a hidden platform (a distal cue) (Rudy, Stadler-Morris \& Albert, 1987). Behavioral results revealed that rats at p17 can only correctly find the platform using proximal cues in the visible platform version of MWM task. Rats at p20 start to show smallest sign of using distal cues in the hidden version of MWM task. These findings suggest that the inability at p17 to correctly use distal cues was likely a result of incompetent spatial learning skills (Rudy, Stadler-Morris \& Albert, 1987).

In another study, the impact of spatial pretraining on hippocampal structure and its association with spatial memory were examined in juvenile LER rats (Keeley, Wartman, Hausler \& Holahan, 2010). Rats (male and female) were trained on a water maze task from p16 to p26 (pretraining group). Later, the pretraining group and a non-pretrained group were trained on p40 to $\mathrm{p} 44$. Results showed that from p19 to p20 both male and female rats significantly improved in finding the hidden platform. When animals were trained at p40, pretrained groups exhibited lower latencies to find the platform compared to the non-pretrained group. At p44, male but not female pretrained rats showed improved memory (Keeley, Wartman, Hausler \& Holahan, 2010).

Immunohistochemical analysis of the same study showed an increase in the synaptophysin staining (a presynaptic terminals marker) in the SO area of the CA3 subregion in the males of pretrained group (Keeley, Wartman, Hausler \& Holahan, 2010). These results suggest that changes in the terminals in the CA3 region may enhance spatial learning and memory in developing rats. 


\section{Overview of the Golgi-Cox technique}

The Golgi method is a technique that is used to impregnate and visualize neurons under light microscopy. The method was discovered and developed by Camillo Golgi, an Italian scientist, in 1873. However, it was applied and used by Santiago y Cajal, a Spanish neuroanatomist, who also improved the technique that is still in use currently (De Carlos \& Borrell, 2007). In 1906, Golgi and Cajal were jointly awarded the Nobel Prize in Medicine and Physiology "in recognition of their work on the structure of the nervous system" (The Nobel Prize).

It can be argued that prior to Golgi and Cajal, the discipline of neuroscience was not well outlined. Early in 1839, scientists Schleden and Schwann proposed the cell theory, suggesting that all body tissues consisted of individual cells. R. Virchow postulated that cells were biologically individual units that determined the functionality of a tissue. Further, Virchow and Remak found that cells come from pre-existing cells by division (De Carlos and Borell, 2007).

The application of the cell theory to nervous tissue was delayed for a half century because of the absence of a good histological method to stain nervous tissue on one hand and the complex architecture of the nervous system on the other. Thus, it was difficult to ascertain whether nerve cells were organized like other cells in the body. In 1871, Josefvon proposed "the reticular theory", stating that the grey matter in the central nervous system was composed of a massive meshed network that composes the nerve fibers leading to the white matter and spinal cord. Camillo Golgi accepted this theory, but he believed that the network was composed of axonal fibers. Eventually, Golgi found a new histological method to impregnate nerve tissues 
and this method significantly improved the understanding of the structure of the nervous system (De Carlos and Borell, 2007).

The Golgi method used by Cajal " involved preparing pieces of nervous tissue 4-5 $\mathrm{mm}$ in thickness, immersed in a solution of potassium dichromate for 1-7 days, to which $1 \mathrm{~g}$ of osmium terroxide was added" (De Carlos and Borell, 2007). Afterwards, pieces were washed twice with $0.75 \%$ aqueous silver nitrate, and they were placed in fresh $0.75 \%$ silver nitrate solution for $1-2$ days at room temperature. Subsequently, the material was embedded in paraffin and $100-250 \mu \mathrm{m}$ thick slices were obtained. Dark residues filled and outlined the nerve cells and processes over a clear yellow background. To describe the view, Cajal stated: "What an unexpected sight! Sparse, smooth and thin filaments or thorny, thick triangular, stellate, or fusiform black cells could be seen against a perfectly translucent yellow background! One might liken the images to Chinese ink drawings on transparent Japanese paper [...] this is the Golgi method" (Cajal, 1899, translated and cited by De Carlos and Borell, 2007).

Cajal started a standardized study of the nervous system using the Golgi method. He discovered that grey matter was not composed of a diffuse network of terminal and collateral fibers. On the other hand, terminal and collateral fibers have free endings, contacting with the cell bodies and dendrites of the surrounding neurons. In the cerebellum, he also found that the axon of some small stellate cells in the molecular layer had free ends over the soma of Purkinje cells, forming pericellular contacts (later named "terminal nests"). Furthermore, he described the mossy fibers of the cerebellum, a type of afferent fibers that establish connections with the small dendrites of the granule cells. He also distinguished a group of very short processes projecting from dendritic surface, and he named them "dendritic spines" (De Carlos and Borell, 2007). 
Since the foundation of the Golgi impregnation method in 1873, neuroscientists have uncovered the mystery of the connections between neurons by sketching the neuronal circuits of the brain (Agnati, Genedani, Leo, Rivera, Guidolin \& Fuxe, 2007). Currently, the Golgi impregnation method remains an important tool in neuroscience, and one of the most used techniques to visualize structural aspects of the central nervous system.

A group of recent studies examined the effect of prenatal, perinatal and postnatal exposure to ethanol that led to distinct morphological changes as revealed by using the Golgi method. The Golgi method was used on a rat model of Fetal Alcohol Spectrum Disorders (FASD) (Lawrence, Otero \& Kelly, 2012). Results revealed that the perinatal ethanol exposure caused morphological changes in dendritic branching, and it reduced spine density in the medial prefrontal cortex and nucleus accumbens (Lawrence, Otero \& Kelly, 2012).

In a similar study, Golgi results showed that prenatal exposure to ethanol produced anomalistic dendritic spines in layer $\mathrm{V}$ pyramidal cells of the parietal cortex in $\mathrm{p} 12$ and $\mathrm{p} 40$ rats (Stoltenburg-Didinger \& Spohr, 1983). In another relevant study, hippocampal CA1 pyramidal neurons deficits were found in $\mathrm{p} 30$ rats exposed to average amounts of ethanol during gestation through the postnatal period. Results revealed a reduced number of pyramidal neurons, spines from surviving neurons, and a reduction in the size of mushroom and ramified spines (GonzálezBurgos, et al., 2006).

Additionally, Golgi studies have been conducted to visualize the effects after experimental exposure of certain neurotoxins on brain during development. The impact of exposure to di(2-ethylhexyl) phthalate (DEHP) during development on the morphology of CA3 pyramidal neurons has been investigated in male and female rats using the Golgi method (Smith 
\& Holahan, 2014). DEHP is a synthetically derived chemical used as plasticizer in a variety of common household products (Holahan \& Smith, 2015). Juvenile male and female rats received injections of DEHP from p16 to p22. Results demonstrated that after DEHP treatment, male (but not female) rats showed a decrease in spine density on the basal and apical dendrites of CA3 pyramidal cells when compared to normal controls. These findings imply a gender-specific susceptibility to early exposure to DEHP in male rats, and that it may prevent normal morphological adjustment in the hippocampus during development (Smith \& Holahan, 2014).

The development of caudate interneurons was investigated after experimental exposure to carbon monoxide in 5 day old rats. Normal spine densities were observed in 6 week old while abnormal growth of the spine numbers was observed at 2-7 months old (Norton \& Culver, 1977). In a related study, rats were injected with fluoxetine (daily for 3 weeks) starting at age p21then were divided into 2 groups; one euthanized after 1 day and the other after 21 days following the last injection. Results showed that dendritic spine density in the second group "remained at levels present in CA1 in p21", suggesting "possible arrest in the development of spines in CA1 into young adulthood" (Norrholm \& Ouimet, 2000).

Researchers also used Golgi impregnation technique for analysing the environmental impacts on the development of the brain. Changes in the neural structure were found in the orbitofrontal cortex (OFC) and medial prefrontal cortex (mPFC) in response to peer play experienced during development. Golgi's method results indicated that the number and length of spines in the OFC increased positively with the number of peers not with the experimental manipulations. However, the number and length of spines in $\mathrm{mPFC}$ increased positively with the complexity of the experience of play (Bell, Pellis \& Kolb, 2010). 
Another study examined the neuronal changes in rat brains after experiencing chronic stress during development (p20 to p41). The Golgi's method results revealed a reduction in dendritic spines in the hippocampus, prefrontal cortex and amygdala (Eiland, Ramroop, Hill, Manley \& McEwen, 2012).

In a further study, Golgi-cox was used to analyse the effect of tactile stimulation of rats during development ( $\mathrm{p} 3$ to $\mathrm{p} 21$ ). The results revealed consequential expansion of dendritic branching, length, and spines density in the prefrontal cortex (mPFC and OFC) and the amygdala (Richards, Mychasiuk, Kolb \& Gibb, 2012). Moreover, in a study that investigated the neural changes in rats weaned on p21 and isolated socially for 8 weeks, Golgi-cox analysis showed a reduction in the branching length and density of the hippocampal CA1 and mPFC pyramidal cells (Silva-Gomez, Rojas, Juarez \& Flores, 2003). Similar results were obtained in another study that investigated the effects of the maternal separation during development (Monroy, Hernández-Torres \& Flores, 2010).

Likewise, scientists have used the Golgi method to study the impact of malnutrition on brain development and results illustrated remarkable changes in the neural characteristics. One study showed that the lack of dietary protein during development had a negative impact on the granule cells of the dentate gyrus of the hippocampal formation. Golgi-impregnated cells revealed a decrease in the number of spines on dendrites in the areas examined in all involved age groups (p30, p90 and p220) (Cintra, Díaz-Cintra, Galván, Kemper \& Kemper, 1990).

Another study was done to analyse the effects of prenatal protein malnutrition on the development of CA3 hippocampal pyramidal cells at ages p15, p30, p90 and p220. Golgi analysis showed a reduction of the soma size at (p90 and p220), apical dendrites at age p15, 
basal at (p15, 90 and 220), and spine density at (p30, 90 and 220) (Díaz-Cintra, García-Ruiz, Corkidi \& Cintra, 1994).

\section{Purpose of the Present Study}

The main purpose of this study was to understand more about learning and memory during development. In particular, the first goal was to investigate the sensitive period of normal morphological changes in the hippocampal CA3 pyramidal neurons. The second goal was to investigate the effect of AMPA receptor antagonist on spatial learning and memory in juvenile rats and the outcomes on the morphology of CA3 pyramidal neurons.

Previous studies have looked at the presynaptic component of the DG-CA3 circuit by investigating the morphological changes of mossy fibers terminals in the CA3 areas during development, and revealed a sensitive morphological adjustment period in juvenile rats (p16p24) (Holahan, Rekart, Sandoval \& Routtenberg, 2006; McGonigal, Tabatadze \& Routtenberg, 2012; Ramírez-Amaya, Balderas, Sandoval, Escobar \& Bermúdez-Rattoni, 2001; Rekart, Sandoval, Bermudez-Rattoni \& Routtenberg, 2007). However, very little is known about the changes in the CA3 pyramidal neurons during the same developing period.

Moreover, this sensitive period (p16-p24) was studied using behavioral tasks i.e. MWM to examine if those morphological modifications are correlated with the ontogeny of spatial ability in rats (Rudy, Stadler-Morris \& Albert, 1987; Keeley, Wartman, Hausler \& Holahan, 2010). Thus, the present study focussed on the postsynaptic component of the DG-CA3 circuit as well as the behavioral changes that emerge during developmental period from p18 to p24.

Experiment 1 examined the morphological changes in CA3 pyramidal neurons during a sensitive developmental period. To achieve this, 16 normally developed LER were used and 
brains were obtained at p18, p20, p22, and p24 ( 4 rats per age group). Brains were impregnated using the Golgi technique to visualize and analyze the differences between ages in the size of the cell body, number and length of apical and basal dendritic branches, the spine density, and the number of TE.

Experiment 2 was designed to test the effect of AMPA receptor antagonist on spatial abilities in juvenile rats, and to analyze the morphological changes of CA3 pyramidal neurons. Eighteen juvenile LER were used, and they were divided to 3 groups: 2 groups were injected with different dosages of (NBQX) AMPA receptor antagonist, and the third group of rats were injected with saline and were used as a control group. Animals received injections before they were trained on the water maze task (for the behavioral analysis) for three days at p18, p19 and $\mathrm{p} 20$, and they were tested on $\mathrm{p} 41$. Brains were obtained for Golgi morphological analysis of CA3 pyramidal neurons after the test.

\section{Methods}

\section{Subjects}

A total of 34 juvenile male Long-Evans rats (LER) were used. Sixteen normal rats were used to examine the structural development of hippocampal CA3 pyramidal neurons at postnatal day 18, 20, 22 and 24 (4 rats per age group). Eighteen rats received either $10 \mathrm{mg} / \mathrm{kg}, 5 \mathrm{mg} / \mathrm{kg}$, or 0mg $/ \mathrm{kg}$ NBQX, AMPA receptor antagonist and were trained in Morris water maze. All subjects were born in the vivarium at Carleton University, and the day the pups were born was considered as $\mathrm{p} 0$. Subjects were housed in a temperature-controlled vivarium in polycarbonate cages with a 12 hour light-dark cycle: lights on $8 \mathrm{~h} 00$, lights off $20 \mathrm{~h} 00$. All rats were group housed with 3 or 2 per cage and food and water provided ad libitum. All experiments were administered in 
conformity with the Canadian Council on Animal Care (CCAC) guidelines and approved by the Carleton University Animal Care Committee.

\section{Morris water maze}

\section{Apparatus}

A white circular pool, 155 in diameter, $60 \mathrm{~cm}$ in height and filled with water to a depth of $37.5 \mathrm{~cm}$ was used. Water temperature was retained at an average of $23^{\circ} \mathrm{C}$ during training and testing. The platform was made of transparent Plexiglas, $14 \mathrm{~cm}$ in diameter, and was immersed 2 $\mathrm{cm}$ below the water surface. Distal visual cues were present surrounding the maze throughout all trails. Rats' movement was tracked using the HVS Image 2100 Tracking System (HVS Image, Buckingham, UK).

\section{Training phase}

Animals $(\mathrm{n}=18)$ were trained in the water maze task on postnatal days 18,19 , and 20 . Prior to training, the rats were divided into 3 groups that received different dosages of NBQX;

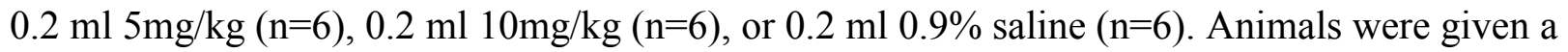
5 minute delay after intraperitoneal (I.P) injections and before training. Rats were trained individually for 8 trials. Each trial was 60 second. During the trials, if a rat did not find the platform after 60 second, they were guided to it.

\section{Testing phase}

Animals were tested on postnatal day 41 . The test was a 60 second trial after removing the platform from the pool. Rats did not receive injections prior to testing. 


\section{Golgi-Cox technique}

Immediately after the probe test, animals were individually removed from the water maze and were administered $0.1 \mathrm{ml}$ Dorminal before they were perfused with $100 \mathrm{ml}$ saline $(0.9 \%)$.

Brains of 12 rats from the pretraining group were obtained and prepared for Golgi impregnation: $5 \mathrm{mg} / \mathrm{kg}$ NBQX (4 rats), 10mg/kg NBQX (4 rats), and 0mg/kg NBQX (4 rats). Brains of normally developed rats: p18 (4 rats), p20 (4 rats), p22 (4 rats) and p24 (4 rats) were removed and prepared for Golgi impregnation.

The Golgi-Cox technique, a modification of the original Golgi, was applied. Instead of the silver nitrate used in the original Golgi method, mercury chloride was used after exposing the tissue to ammonia to gain dark mercury residue. The Golgi fix was prepared by mixing the solutions (A, B and C) as follows. Solution A: Mercuric Chloride $\left(\mathrm{HgCl}_{2}\right), 37.5 \mathrm{~g}$ and hot $\mathrm{DH}_{2} \mathrm{O}$ $\left(95^{\circ} \mathrm{C}\right), 750 \mathrm{ml} . \mathrm{HgCl} 2$ was added and stirred to dissolve in the $950 \mathrm{C}$ heated water. Then, the solution was allowed to cool while preparing solutions B and C. Solution B: Potassium Dichromate $\left(\mathrm{K}_{2} \mathrm{Cr}_{2} \mathrm{O}_{7}\right), 37.5 \mathrm{~g}$ and warm $\mathrm{DH}_{2} \mathrm{O}\left(60^{\circ} \mathrm{C}\right), 750 \mathrm{ml}$. Water was heated to $60^{\circ} \mathrm{C}$ and then removed to another stir plate (without heating). $\mathrm{K} 2 \mathrm{Cr} 2 \mathrm{O} 7$ was added and was let to dissolve. Solution was allowed to cool while preparing solution C. Solution C: Potassium Chromate $\left(\mathrm{K}_{2} \mathrm{Cr}_{2} \mathrm{O}_{4}\right), 30.0 \mathrm{~g}$ and $\mathrm{DH}_{2} \mathrm{O}, 750 \mathrm{ml}$. $\mathrm{K} 2 \mathrm{Cr} 2 \mathrm{O} 4$ was added and dissolved in water at room temperature.

Solutions A, B and C were mixed in a new flask. $1500 \mathrm{ml} \mathrm{DH2O} \mathrm{(room} \mathrm{temperature)} \mathrm{was}$ added, yielding to Golgi fix, an "orangey-rust” final product. In consideration of the sensitivity of Golgi fix, it was transferred to a brown glass jar covered with tinfoil and stored in the dark for 
at least 6 nights prior to use. Brains were placed in Golgi fix solution in vials covered with tinfoil immediately after they were removed.

After the incubation period (4 days), brains were washed following the Golgi-Cox Mercury Impregnation Method Protocol, starting with rinsing brain in 3 changes of DH2O: first, brains were left for 4 hours, the second for 3 hours, and the last one overnight. Then, brains were washed in $10 \%$ sucrose and were kept for 8 hours. Next, brains were kept in $20 \%$ sucrose overnight. Finally, brains were kept in 30\% sucrose for a minimum of 4 days.

Following fixation, brains were cut using Vibratome, an apparatus used for cutting through brain tissue by vibrating blade in a chamber filled with $10 \%$ sucrose. Sections at $200 \mu \mathrm{m}$ thickness were obtained and mounted immediately onto double dipped gelatinized slides. A piece of parafilm were placed on top of sections, Kim wipe (dipped in 10\% sucrose) was placed over it, and another microscope slide was placed above it and was pressed gently to remove excess water. Slides were placed in humidified box in dark cupboard for 24 hours.

For impregnation, slides were put in slide container and submerged through following solutions (in the same order): $\mathrm{dH} 2 \mathrm{O}$ (1 min), 28\% Ammonium Hydroxide (40 min), dH2O (1 min), Kodak film fix A (diluted 1:1 with dH2O) (40 min), dH2O (1 min), dH2O (1 min), 50\% ETOH (1 min), 70\% ETOH (1 min) and 95\% ETOH (1 min). From this point onwards all alcohols and clearenes were desiccated with type 3 Amolecular sieve, 1/16" pellets for at least 24 hours: $100 \% \mathrm{ETOH}(5 \mathrm{~min}), 33 \% \mathrm{ETOH}, 33 \%$ clearene, $33 \%$ chloroform (10 min) and clearene (15 min). After impregnation, slides were coversliped in fumehood using Permount and placed in desiccated box for a minimum of 3 days to allow the Permount to harden before analysis. 
Golgi-impregnated neurons were randomly selected for tracing from a population of hippocampal CA3 pyramidal neurons following criteria: a visibly complete neuron (not cut due to sectioning), dark and consistent impregnation throughout the entire dendrites and relatively isolated from nearby impregnated neurons.

For Experiment 1, a CA3 neuron from each rat was selected for reconstruction and structural analysis: $\mathrm{p} 18(\mathrm{n}=4), \mathrm{p} 20(\mathrm{n}=4), \mathrm{p} 22(\mathrm{n}=4)$ and $\mathrm{p} 24(\mathrm{n}=4)$. Two CA3 pyramidal neurons from each rat were chosen for TE quantification: p18 $(\mathrm{n}=8), \mathrm{p} 20(\mathrm{n}=8), \mathrm{p} 22(\mathrm{n}=8)$ and $\mathrm{p} 24(\mathrm{n}=8)$.

Similarly, for Experiment 2, a CA3 neuron from each rat was selected for reconstruction and structural analysis: $0 \mathrm{mg}(\mathrm{n}=4), 5 \mathrm{mg}(\mathrm{n}=4)$ and $10 \mathrm{mg}(\mathrm{n}=4)$. For TE quantification, two CA3 pyramidal neurons from each rat were chosen: $0 \mathrm{mg}(\mathrm{n}=8), 5 \mathrm{mg}(\mathrm{n}=8)$ and $10 \mathrm{mg}(\mathrm{n}=8)$.

Neurons were traced using Neurolucida software (MBF Biosciences) and Olympus BX51 microscope with 100X oil objective. Neurolucida Explorer (MBF Biosciences) was used to obtain data on CA3 pyramidal neuron's different elements and measurements as shown in the result section of this thesis.

For thorny excrescences (TE) quantification, three criteria based on Gonzales, et al., (2001) study were used. First, counting thorny excrescences covered at least two thirds of the length of apical or basal dendrites starting from the base of the dendrites on the cell body to the end of the branches. Second, a TE had to be clearly impregnated, dark and have a clear neck attached to the dendrite. Third, only complete neurons that are not cut during sectioning were chosen for TE quantification. In addition, only neurons in the midfield of CA3 (that have their apical dendrites in the SL and basal dendrites in the SO) were used. 
Selected CA3 pyramidal neurons showed two main types of spines. Shorter and smaller spines ( 0.5 to $2 \mu \mathrm{m}$ emerged out of a dendrite) were seen on all apical and basal dendrites and were similar to the spines on CA1 pyramidal neurons (Harris, Jensen \& Tsao, 1992). The second type was excrescences that are only seen on CA3 pyramidal neurons.

Under the 100X lens of the light microscopy, a single excrescence shows a neck that emerges from a dendrite and divides into several heads (two or more). According to Gonzales, et al., (2001), a head on a single TE can be between $0.57 \mu \mathrm{m}$ and $6.24 \mu \mathrm{m}$. A single TE can also extend around $4.5 \mu \mathrm{m}$ on a dendrite (Chicurel and Harris, 1992).

Reconstructions of the neurons and TE quantification in Exp.1 and Exp.2 are done blindly throughout the study. Groups were revealed following statistical analysis.

\section{Results}

\section{Experiment 1}

Sixteen juvenile rats were used for Experiment 1. Rats were divided into 4 age groups (4 rats per group) and sacrificed at $\mathrm{p} 18, \mathrm{p} 20, \mathrm{p} 22$ or $\mathrm{p} 24$. Brains were obtained for structural analysis of CA3 pyramidal neurons using Golgi's method (see Figure 2). Measurements included: cell body area, number of apical and basal dendrites, number of branch nodes and ends, length, surface, volume and complexity. In addition, spines and thorny excrescence quantifications were provided. 

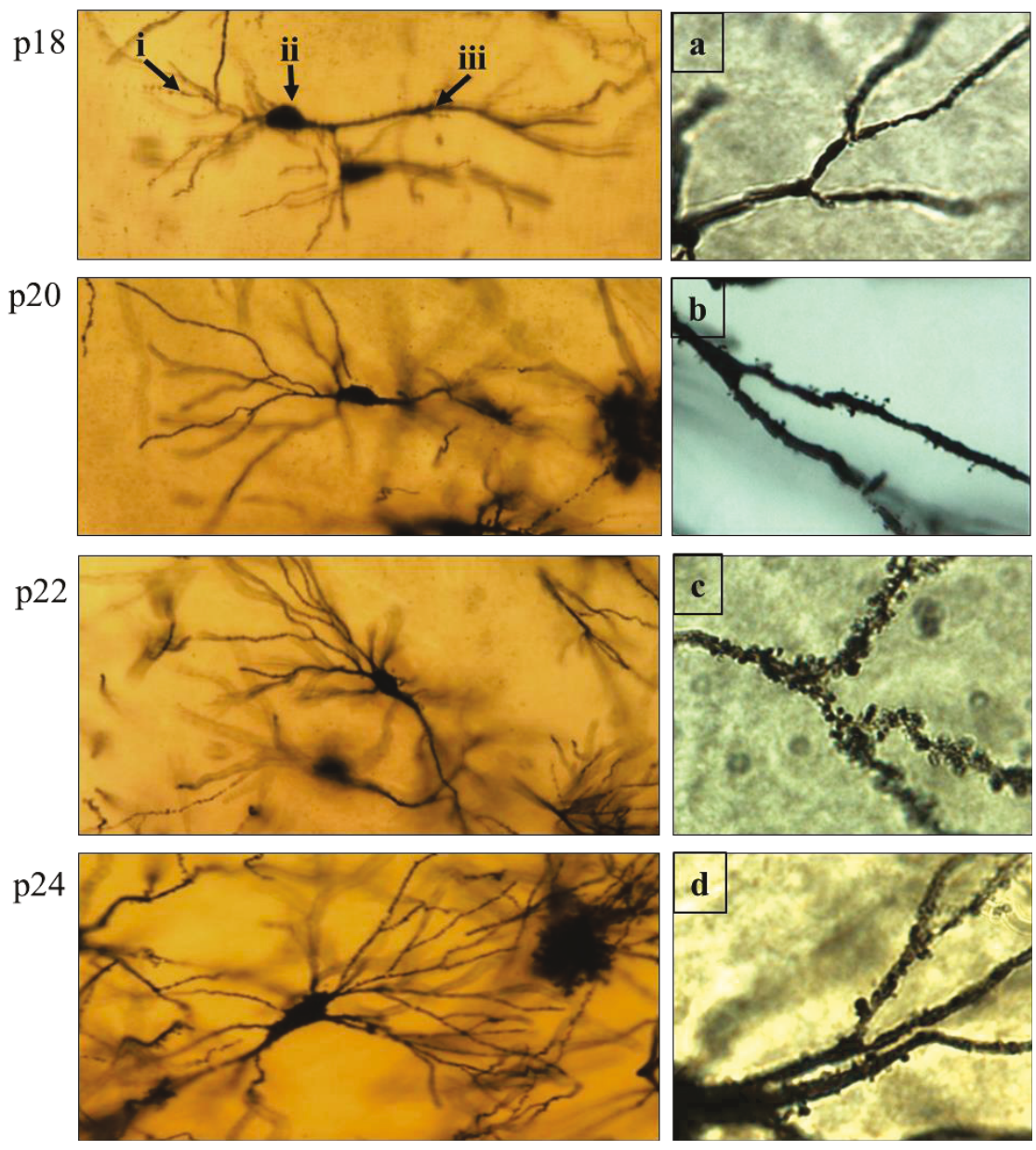

Figure 2. Representative images for Golgi impregnation of CA3 pyramidal neurons at $\mathrm{p} 18$, p20, p22 and p24 taken at 20X. Images: a, b, c and $\mathbf{d}$ represent spines on the basal dendrites of CA3 neurons at p18, p20, p22 and p24 respectively. Images were taken at 100X. Arrows: i. Basal dendrites. ii. Cell body. iii. Apical dendrites.

\section{CA3 structural analysis}

\section{Cell body area $\left(\mu \mathrm{m}^{2}\right)$}

A one-way analysis of variance ANOVA was conducted to determine if the cell body area of CA3 pyramidal neurons was different between age groups. Figure 3 shows that the area of the cell body slightly increased at p22 and p 24 , but the difference was not statistically significant, $\left(F_{(3,12)}=1.03, p=0.412\right)$. 


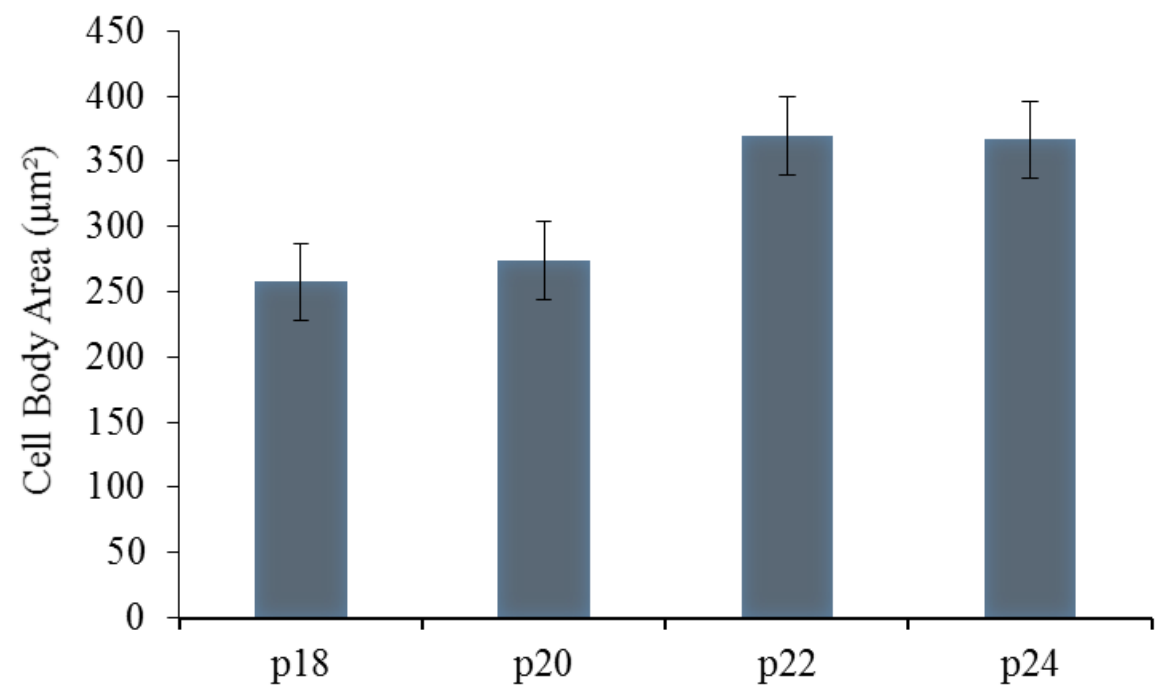

Figure 3. Area of the cell body of CA3 pyramidal neurons measured at p18, p20, p22 and p24. No main effect of age was found.

Number of dendrites

A one-way ANOVA on the number of basal dendrites across the age groups, shown in Figure 4 , revealed a main effect of age $\left(F_{(3,12)}=4.25, p<0.05\right)$. Tukey post-hoc tests revealed significantly fewer basal dendrites between p20 and p24 $(p<0.05)$. 


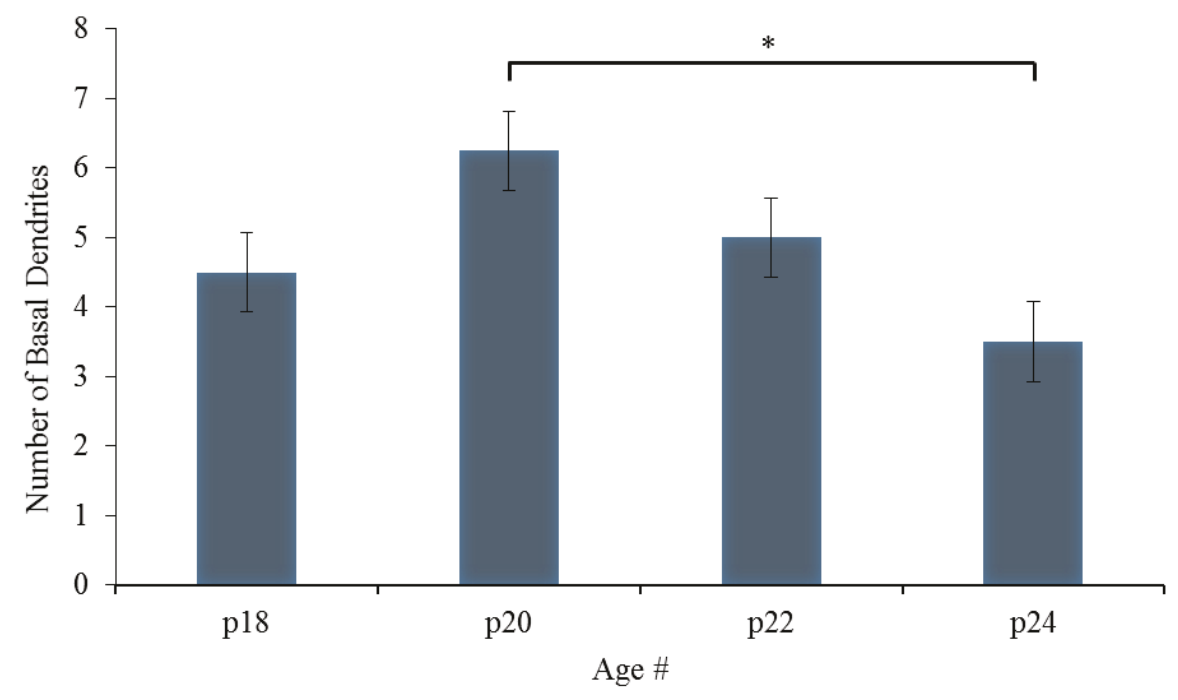

Figure 4. Quantification of basal dendrites of the CA3 pyramidal neurons at: p18, p20, p22, and p24. (\#) indicates a main effect of age $(p<0.05) ;(*)$ indicates a significant difference using Tukey post-hoc tests between $\mathrm{p} 20$ and $\mathrm{p} 24(p<0.05)$.

Number of apical dendrites, as shown in Figure 5, increased gradually with age from p18 $(m=1, s=.00)$ to $222(m=2, s=0.81)$, and then decreased at $\mathrm{p} 24(m=1.75, s=0.95)$.One-way ANOVA did not reveal a statistically significant difference between age groups, $\left(F_{(3,12)}=1.81, p\right.$ $=0.197)$. 


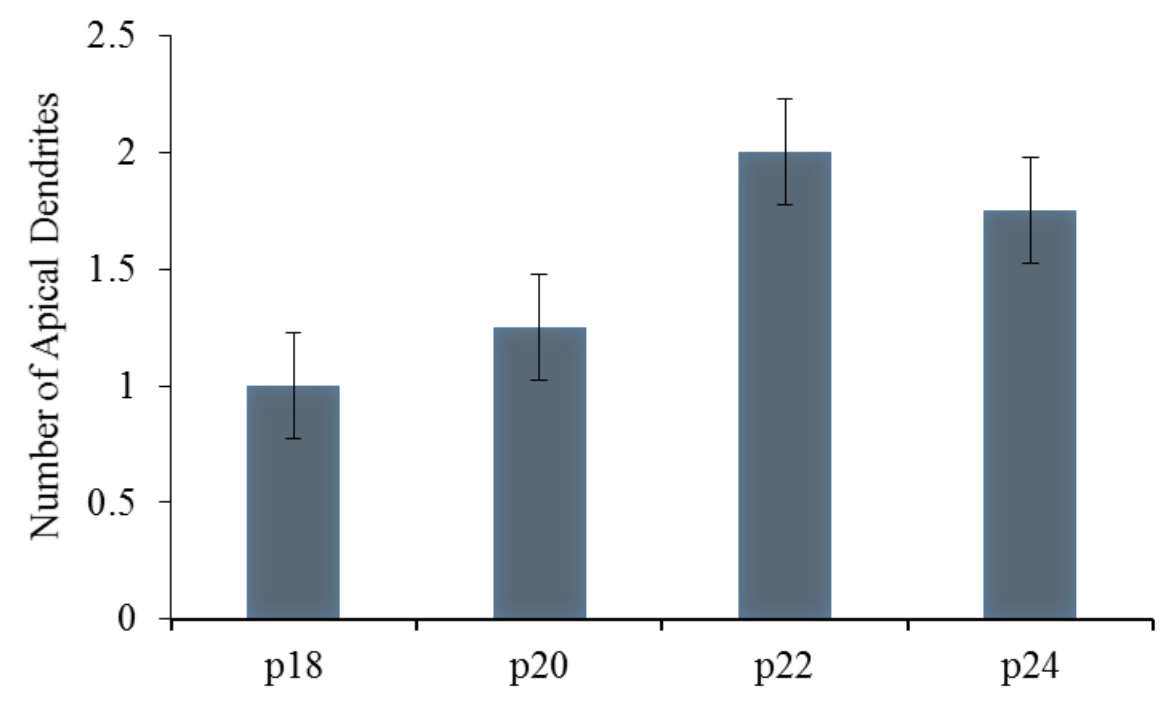

Figure 5. Quantification of apical dendrites of $\mathrm{CA} 3$ pyramidal neurons at different ages were compared and no main effect of age was found.

Branch nodes and ends

Number of nodes in the apical and basal dendrites, plotted in Figure 6, increased sharply from $\mathrm{p} 18(m=19.50, s=3.31)$ to $\mathrm{p} 20(m=41, s=16.41)$, and then remained relatively level at p22 $(m=39.75, s=13.57)$ to $24(m=33.50, s=15.80)$. No statistically significant difference between the groups was found using one-way ANOVA, $\left(F_{(3,12)}=2.17, p=0.144\right)$. 


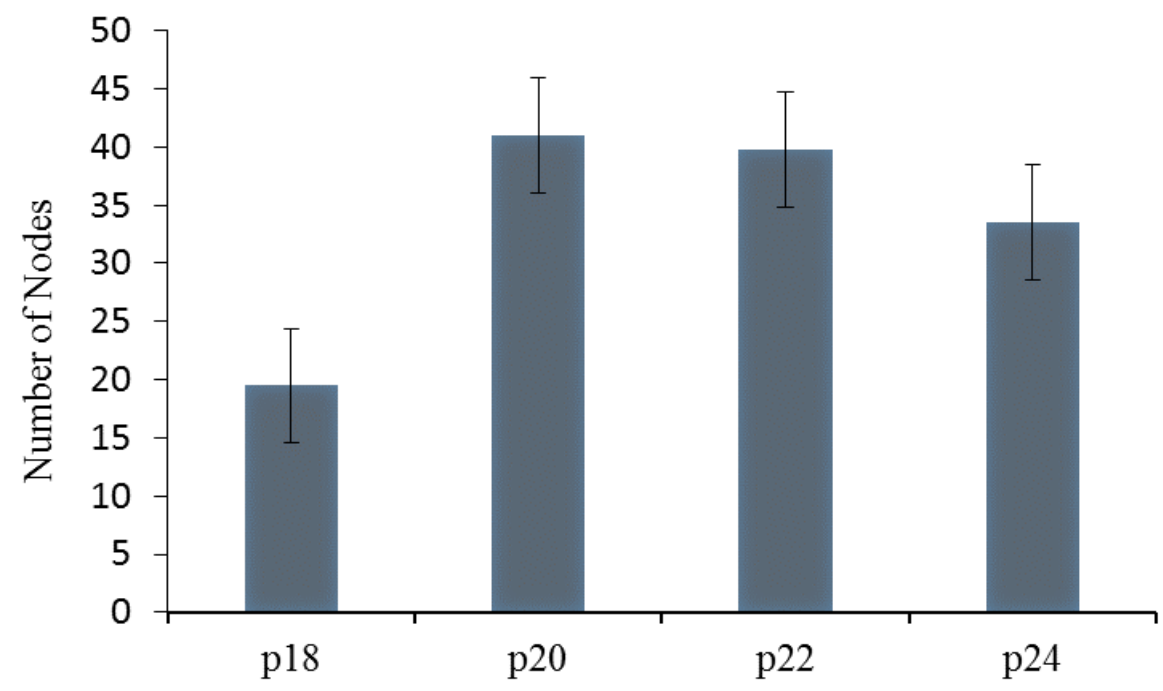

Figure 6. Quantification of nodes in the CA3 pyramidal neurons were compared between age groups and no main effect of age was found.

Quantification of branch ends of apical and basal dendrites, plotted in Figure 7, increased sharply from p18 $(m=27.25, s=1.70)$ to $20(m=54, s=14.8)$, then slightly decreased with age at p22 $(m=50.5, s=16.13)$ to $\mathrm{p} 24(m=42.5, s=18.19)$. No statistically significant difference between the groups was found using one-way ANOVA, $\left(F_{(3,12)}=2.77, p=0.087\right)$. 


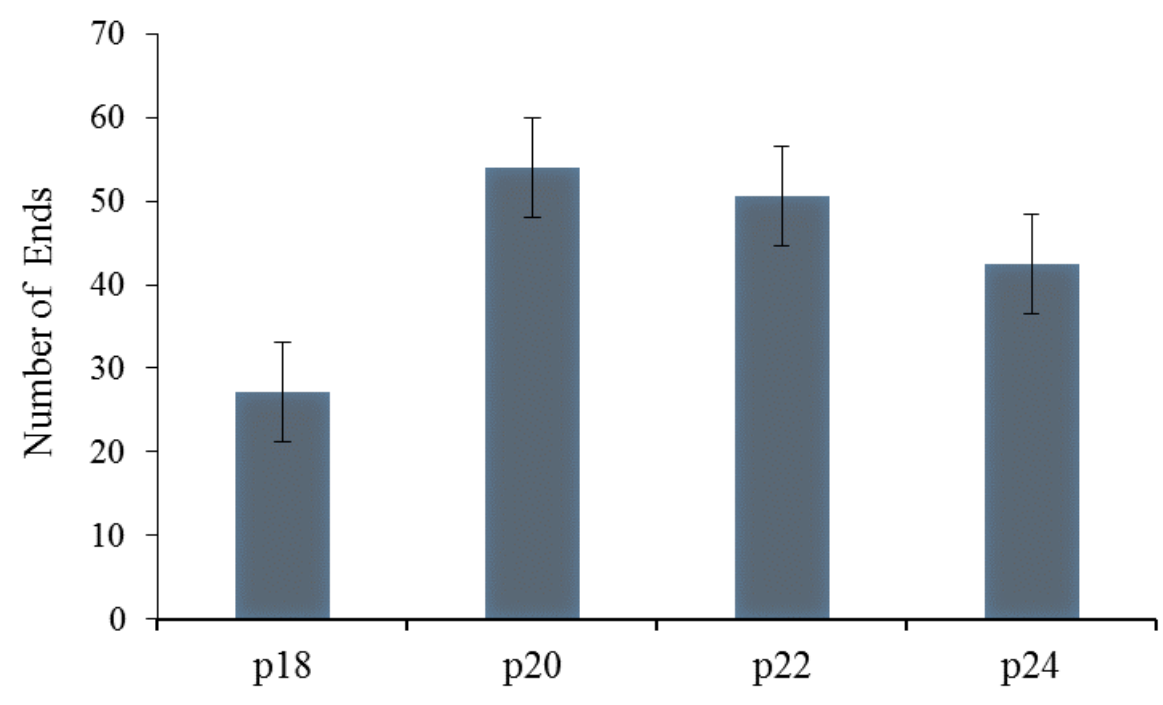

Figure 7. Quantification of ends of apical and basal branches in the CA3 pyramidal neurons were compared between age groups and no main effect of age was found.

\section{Length $(\mu \mathrm{m})$}

A Welch ANOVA was conducted to determine if length of CA3 pyramidal neuron was different between age groups: p18, p20, p22 and p24, plotted in Figure 8. Welch ANOVA is used to test if there is a difference in means between groups when the homogeneity of variances of the dependent variable (in this case the length of the neuron) is violated. A main effect of age was found $\left(F_{(3,5.06)}=6.09, p<0.05\right)$. The length increased gradually with age: $\mathrm{p} 18(m=971.95, s=$ 186.85), p20 $(m=1549.25, s=972.94), \mathrm{p} 22(m=2873.97, s=1669.41)$ and reached the peak at p24 ( $m=4271.6, s=3953.3)$. However, post-hoc for all possible pairwise comparisons using Games-Howell tests revealed no statistically significant differences between age groups. 


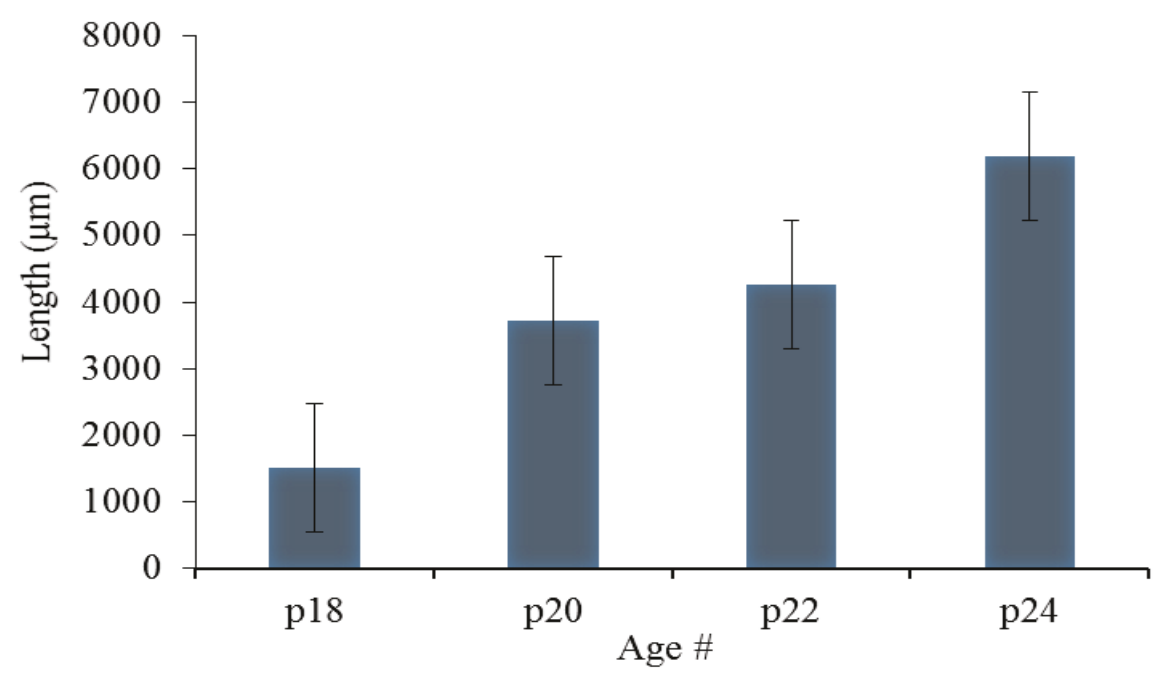

Figure 8. Length of the CA3 pyramidal neurons measured in $(\mu \mathrm{m})$. (\#) indicates a main effect of age $(p<0.05)$.

For a further investigation, one-way ANOVA was conducted to determine if there was a difference between groups in the length of apical and basal dendrites. As shown in Figure 9a, the length of apical dendrites appeared to differ with age. Length was at the lowest at p18 $(\mathrm{m}=$ $485.87, s=198.58)$, and it was almost tripled at p20 $(m=2103.17, s=1138.73)$. Then, length decreased at p22 $(m=1295.2, s=890.74)$ and increased again at p24 $(m=1805.25, s=1068.03)$. The difference between groups was not statistically significant, $\left(F_{(3,12)}=2.45, p=0.144\right)$.

Length of basal dendrites, plotted in Figure 9b, increased gradually with age: p18 ( $m=$ $1512.7, s=152.03), \mathrm{p} 20(m=3725.77, s=1335.33), \mathrm{p} 22(m=4258.77, s=1848.93)$, and 24 ( $m=6181.32, s=4834.62)$. The difference between groups was not statistically significant $\left(F_{(3,}\right.$ 5.16), $p=0.180)$. 

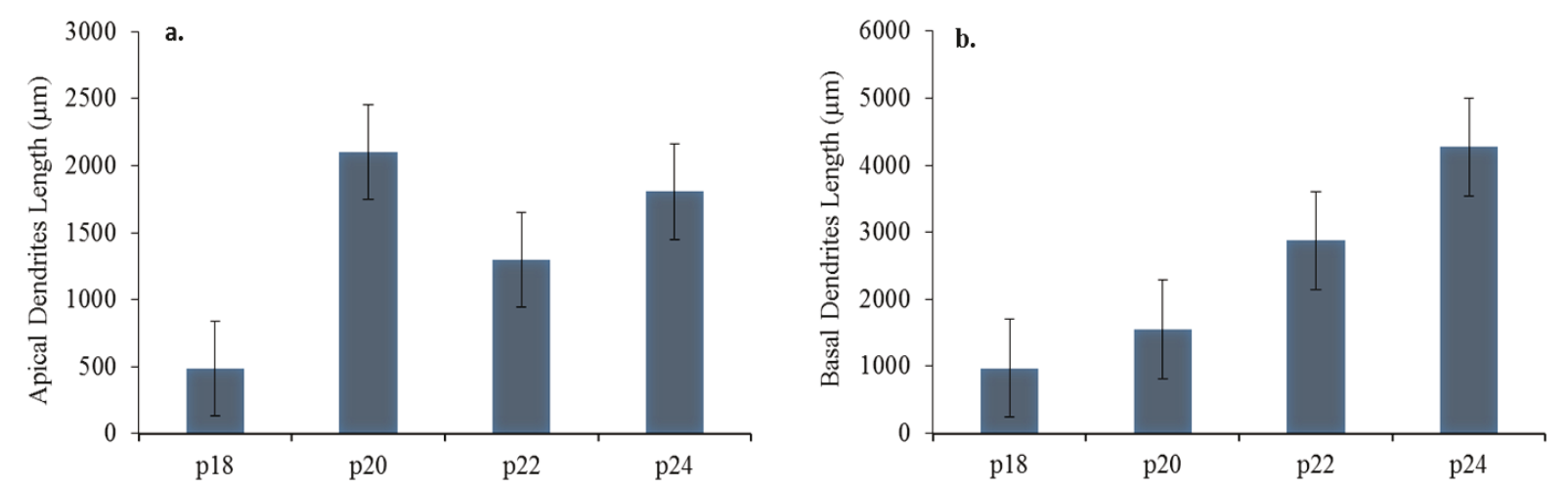

Figure 9. a. Length of the apical dendrites of the CA3 pyramidal neurons measured in $(\mu \mathrm{m})$; no main effect of age was found. b. Length of the basal dendrites of the CA3 pyramidal neurons measured in $(\mu \mathrm{m})$; no main effect of age was found.

\section{Surface, volume and complexity:}

Surface of the CA3 pyramidal neurons, plotted in Figure 10a, increased with age: p18 ( $m$ $=330.25, s=40.27), \mathrm{p} 20(m=827.39, s=300.37), \mathrm{p} 22(m=944.46, s=413.13)$, and $\mathrm{p} 24(m=$ 1376.60, $s=1098.46)$. Welch ANOVA revealed a main effect of age $\left(F_{(3,5.09)}=6.02, p<0.05\right)$. However, pairwise comparisons using Games-Howell post-hoc tests revealed no statistically significant difference between the groups.

Volume of the CA3 pyramidal neurons, plotted in Figure 10b, showed a similar trend as shown by surface. Volume increased with age: p18 $(m=5.95, s=0.72), \mathrm{p} 20(m=14.91, s=5.4)$, p22 $(m=17.02, s=7.44)$, and p24 $(m=24.81, s=19.80)$. A main effect of age was found, $\left(F_{(3,5.09)}=6.02, p<.05\right)$. No statistically significant differences were found between groups when the Games-Howell post hoc test was used. 


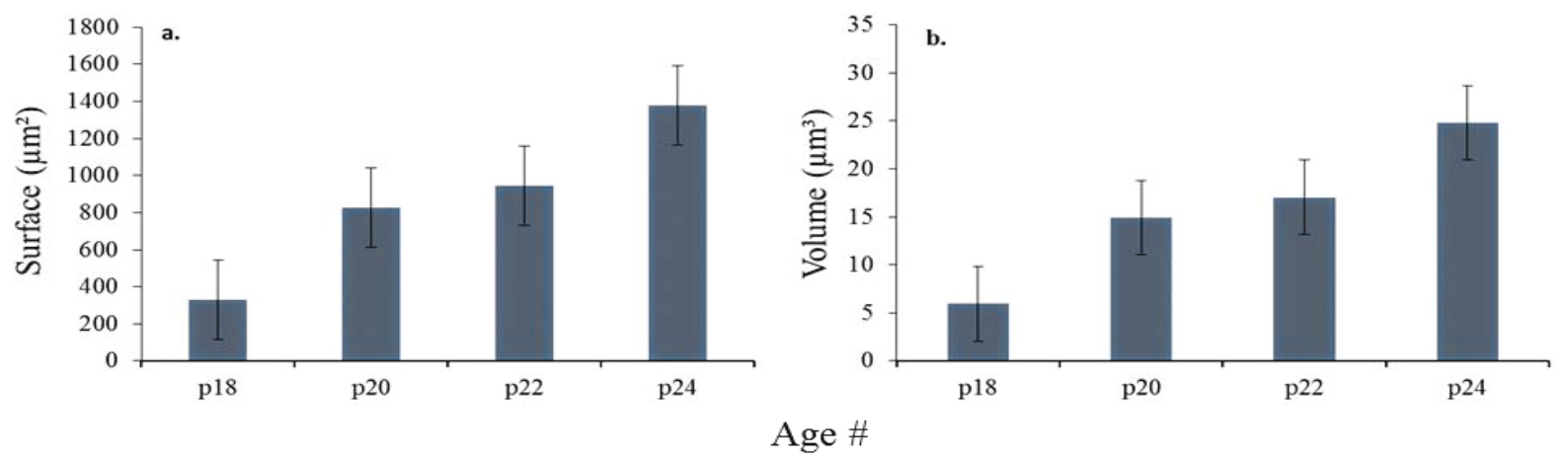

Figure 10. a. Surface of the CA3 pyramidal neurons measured in $\left(\mu \mathrm{m}^{2}\right)$. b. Volume of the CA3 pyramidal neurons measured in $\left(\mu \mathrm{m}^{3}\right)$. (\#) indicates a statistically significant effect of age in both surface and volume $(p<0.05)$.

Complexity, shown in Figure 11, differed between age groups. Complexity increased dramatically from p18 $(m=47799.80, s=38266.73)$, to $\mathrm{p} 20(m=319322.80, s=303612.85)$, dropped at p22 $(m=202250.49, s=111097.88)$, and increased again at p24 $(m=266729.85, s=$ 207960.16). However, one-way ANOVA revealed no statistically significant difference between age groups $\left(F_{(3,12)}=1.484, p=0.268\right)$. 


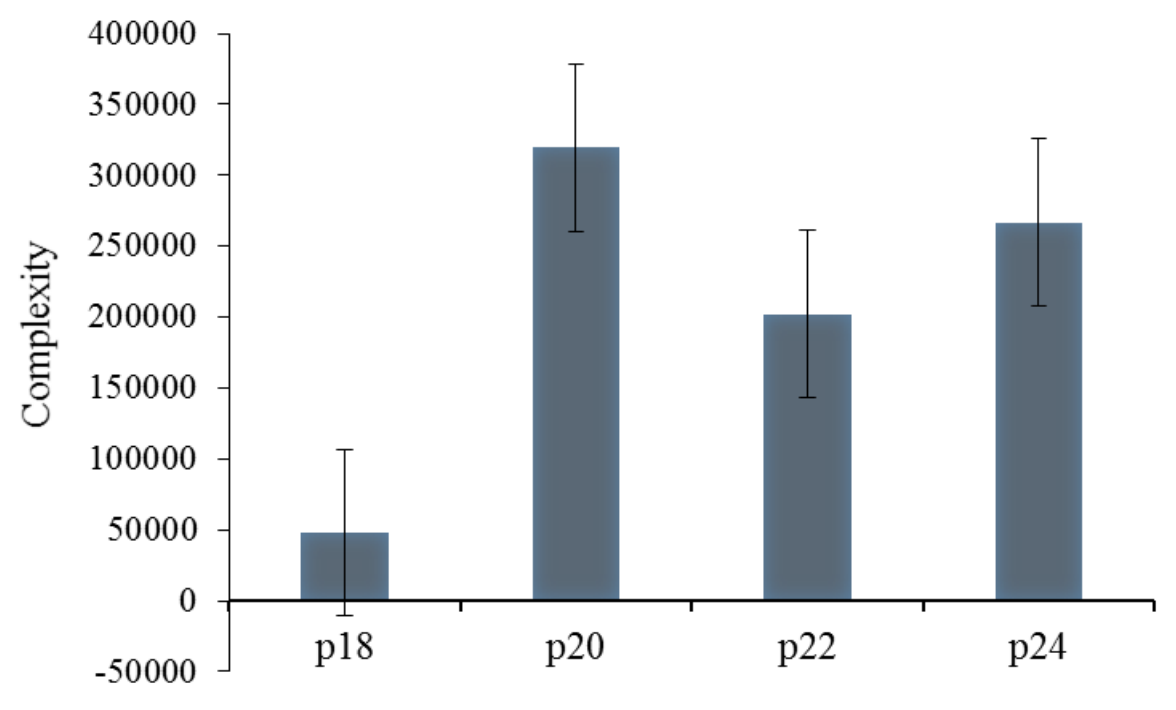

Figure 11. Complexity of the CA3 pyramidal neurons was measured and no main effect of age was found.

\section{Spine quantification}

\section{Entire neuron}

A one-way ANOVA was conducted to determine if there was a change in spine number or density in the entire CA3 pyramidal neuron during development. Total number of spines, plotted in Figure 12, increased gradually with age from p18 $(m=467.75, s=159.52), \mathrm{p} 20(m=$ $1132, s=409.17)$, to $222(m=1563.25, s=653.29)$ then declined at p24 $(m=1020, s=662.87)$. However, no statistically significant difference between age groups was found, $\left(F_{(3,12)}=3.07, p=\right.$ $0.069)$. 


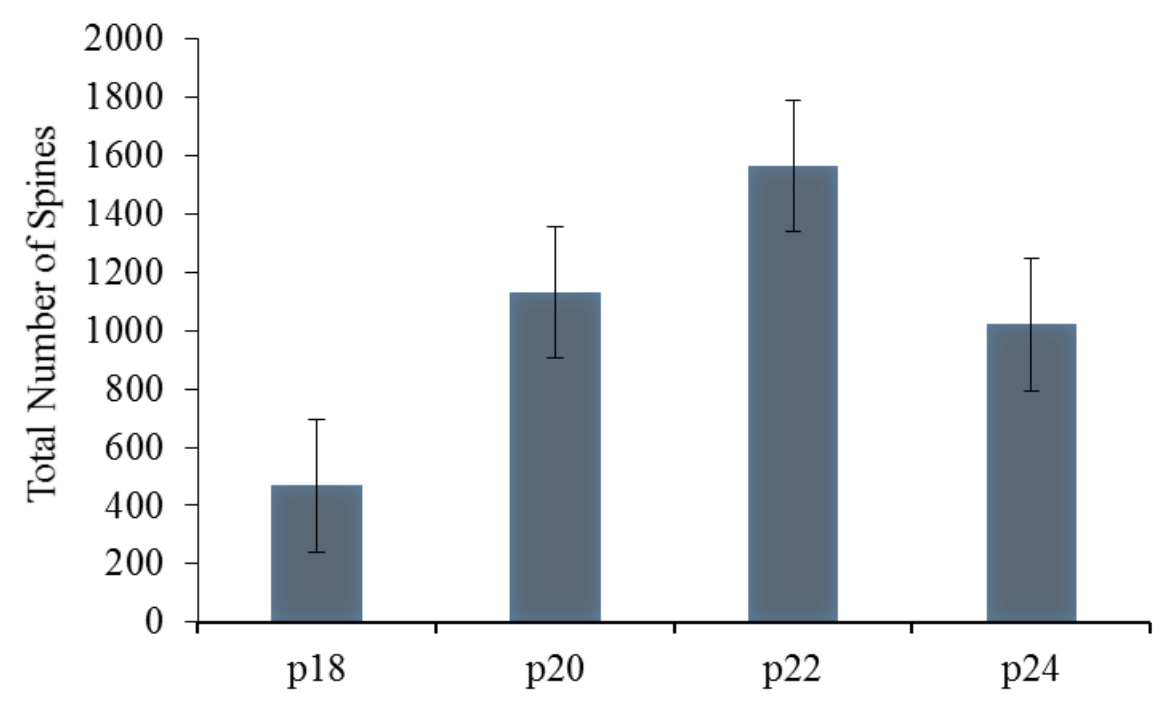

Figure 12. Quantification of the total number of spines on CA3 pyramidal neurons. No main effect of age was found.

Total spine density, measured as the total number of spines divided by the total length of the neuron, was obtained and plotted in Figure 13. One-way ANOVA revealed a main effect of age $\left(F_{(3,12)}=3.846, p<0.03\right)$. Tukey post-hoc tests revealed a significant difference between $\mathrm{p} 22$ $(m=0.38, s=0.11)$ and $\mathrm{p} 24(m=0.19, s=0.06)(p<0.05) . \mathrm{P} 18$ and $\mathrm{p} 20$ spine density were similar $(m=0.31, s=.07)$ and $(m=0.31, s=.05)$ respectively, and reached the highest point at p22. 


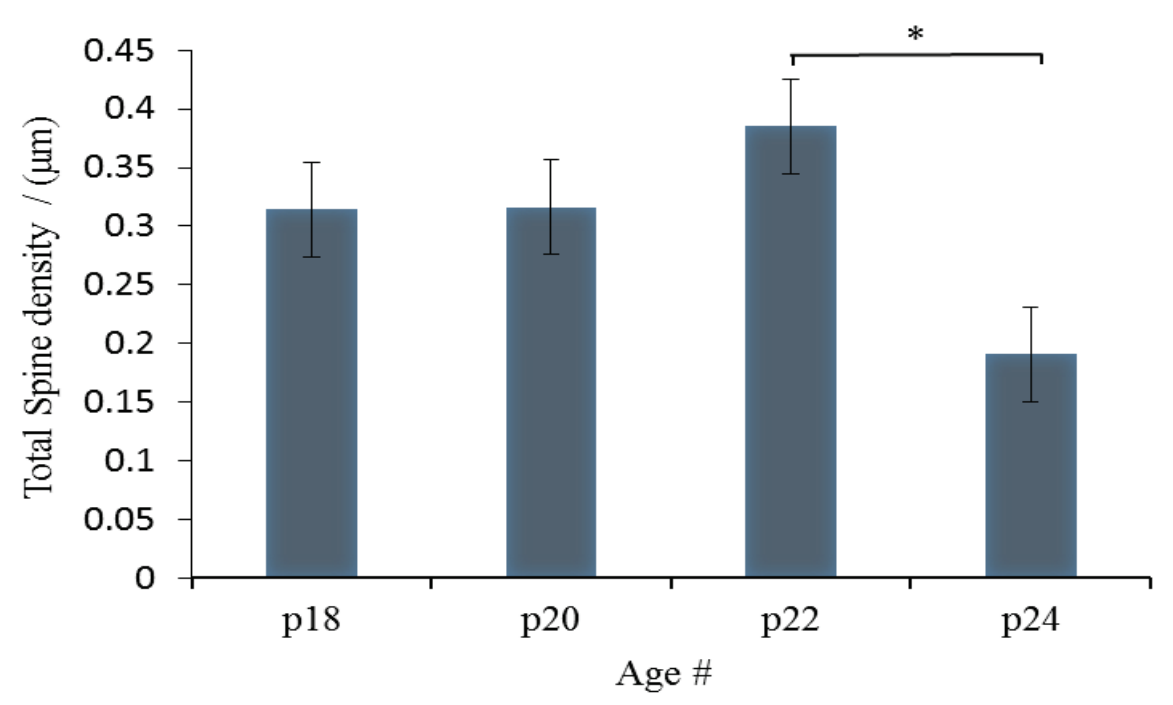

Figure 13. Total spine density per $\mu \mathrm{m}$ of $\mathrm{CA} 3$ pyramidal neurons at: $\mathrm{p} 18, \mathrm{p} 20, \mathrm{p} 22$, and p24.

$(\#)$ indicates a main effect of age $(p<0.05) ;\left(^{*}\right)$ indicates a significant difference using Tukey post-hoc tests between p22 and p24 $(p<0.05)$.

Total spine density was obtain by taking the total number of spines divided by the number of dendrites (both apical and basal; Figure 14). The total density increased with age from p18 $(m=90.67, s=37.27), \mathrm{p} 20(m=157.53, s=76.28)$, to $222(m=219.86, s=84.21)$. Spine density slightly decreased at p24 $(m=194.32, s=114.02)$. One-way ANOVA revealed no statistically significant difference between the age groups $\left(F_{(3,12)}=1.84, p=0.193\right)$. 


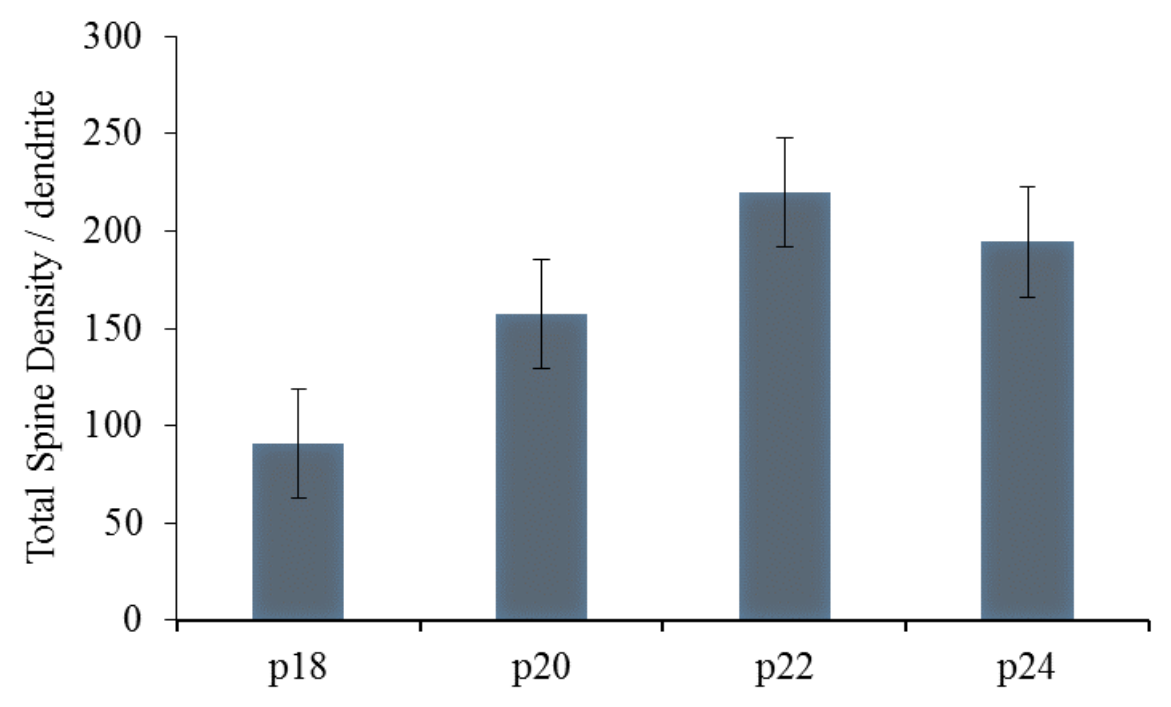

Figure 14. Total spine density per dendrite (apical or basal) of CA3 pyramidal neurons was quantified and no main effect of age was found.

\section{$\underline{\text { Apical spines }}$}

One-way ANOVA was conducted to determine if there is a difference between age groups in the spine number or density on the apical dendrites of CA3 pyramidal neurons. Apical spine number, plotted in Figure 15, increased dramatically from p18 $(m=164.5, s=89.55)$ to p20 $(m=638.25, s=392.47)$. Subsequently, spine number decreased steadily with age: p22 ( $m=$ 342.75, $s=270.26)$ and p24 $(m=311.5, s=211.78)$. No statistically significant difference was found between the groups $\left(F_{(3,12)}=2.25, p=0.135\right)$. 


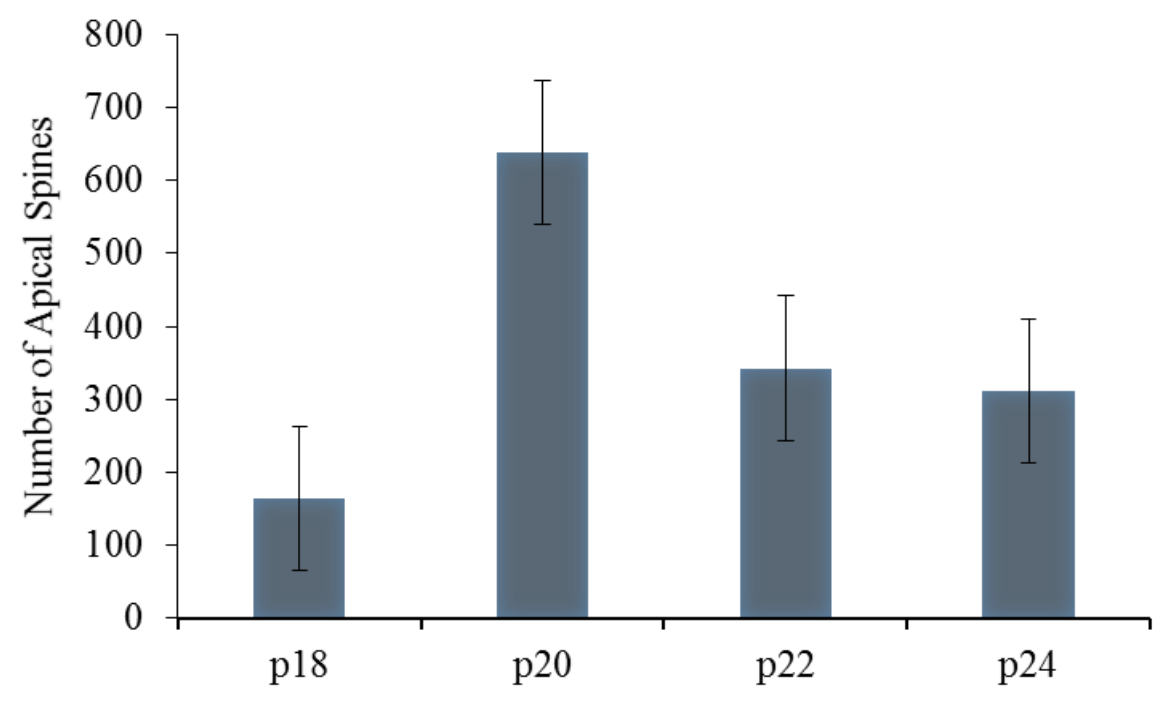

Figure 15. Quantification of spines on the apical dendrites of CA3 pyramidal neurons. No main effect of age was found.

Apical spine density, measured as the number of spines on the apical dendrites divided by length of the apical dendrites of CA3 pyramidal neurons, was obtained and plotted in Figure 16. Apical spine density was at the highest at $\mathrm{p} 18(m=0.32, s=0.10)$. It decreased gradually after that: $\mathrm{p} 20(m=0.31, s=0.11), \mathrm{p} 22(m=0.27, s=0.11)$ and $\mathrm{p} 24(m=0.15, s=0.05)$. However, one-way ANOVA revealed no statistically significant difference between age groups $\left(F_{(3,12)}=\right.$ $2.55, p=0.104)$. 


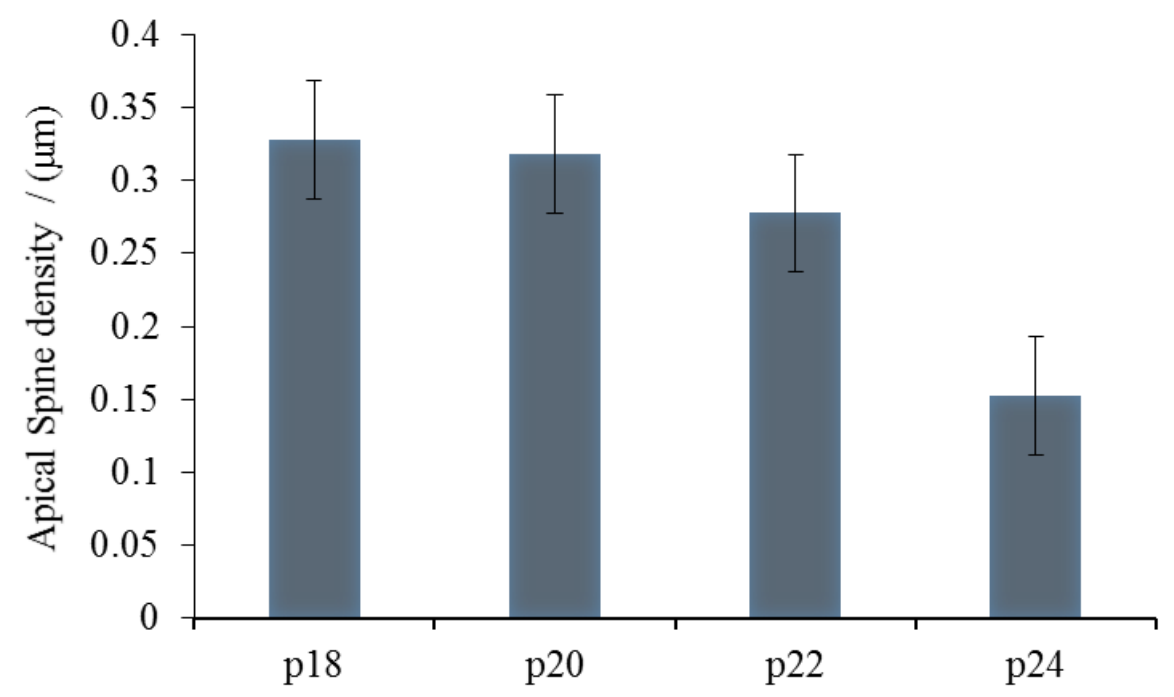

Figure 16. Spine density per $\mu \mathrm{m}$ of the apical dendrites of CA3 pyramidal neurons was quantified and no main effect of age was found.

Apical spine density was obtained by measuring the number of spines on the apical dendrites divided by the number of apical dendrites (Figure 17). The total density increased vastly from $\mathrm{p} 18(m=164.4, s=89.55)$ to $\mathrm{p} 20(m=499.12, s=235.21)$. The density dropped at p22 $(m=244.5, s=308.5)$ and further declined at p24 $(m=185.62, s=138.39)$. One-way ANOVA revealed no statistically significant difference between the age groups $\left(F_{(3,12)}=2.14, p\right.$ $=0.148)$. 


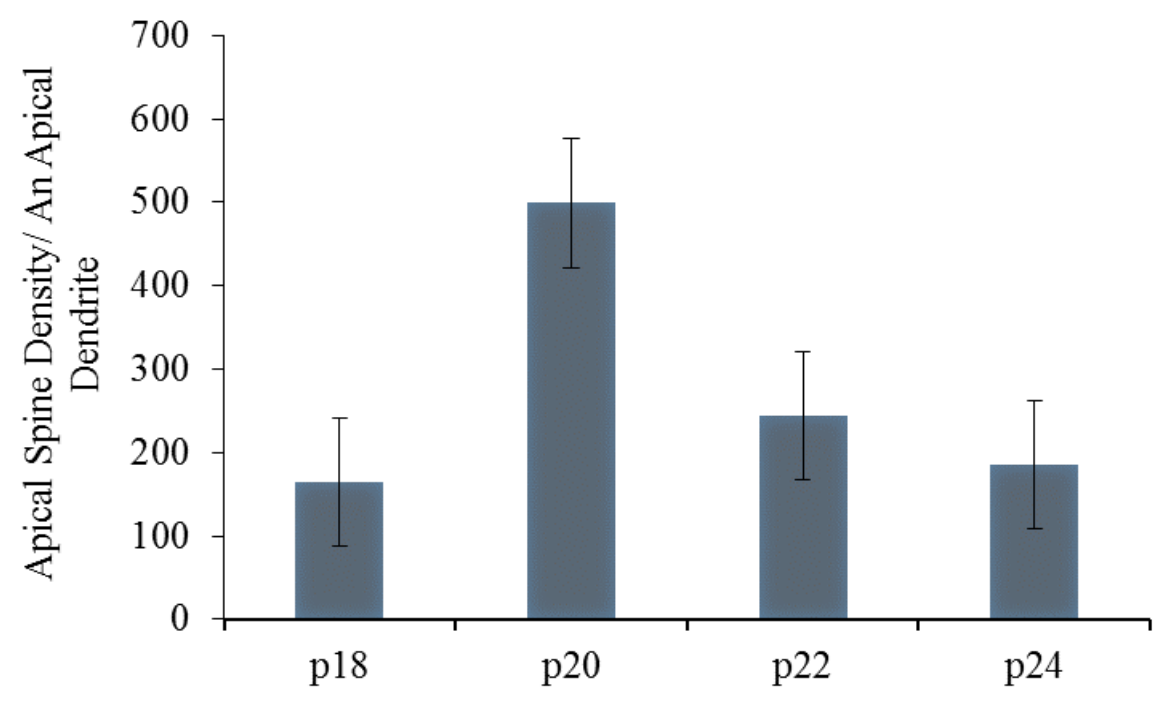

Figure 17. Spine density per apical dendrite of $\mathrm{CA} 3$ pyramidal neurons was quantified and no main effect of age was found.

Basal spines:

A one-way ANOVA was conducted to determine if there was a difference between age groups in spine number and density on the basal dendrites of CA3 pyramidal neurons. Basal spine number, plotted in Figure 18, increased slightly from p18 $(m=303.25, s=117.77)$ to $\mathrm{p} 20$ $(m=493.75, s=298.89)$, and then it increased considerably at $\mathrm{p} 22(m=1220.5, s=799.06)$. Subsequently, the number of spines dropped at p24 ( $m=708.50, s=547.74)$. However, no statistically significant difference was found between the age groups $\left(F_{(3,5.58)}=2.14, p=0.202\right)$. 


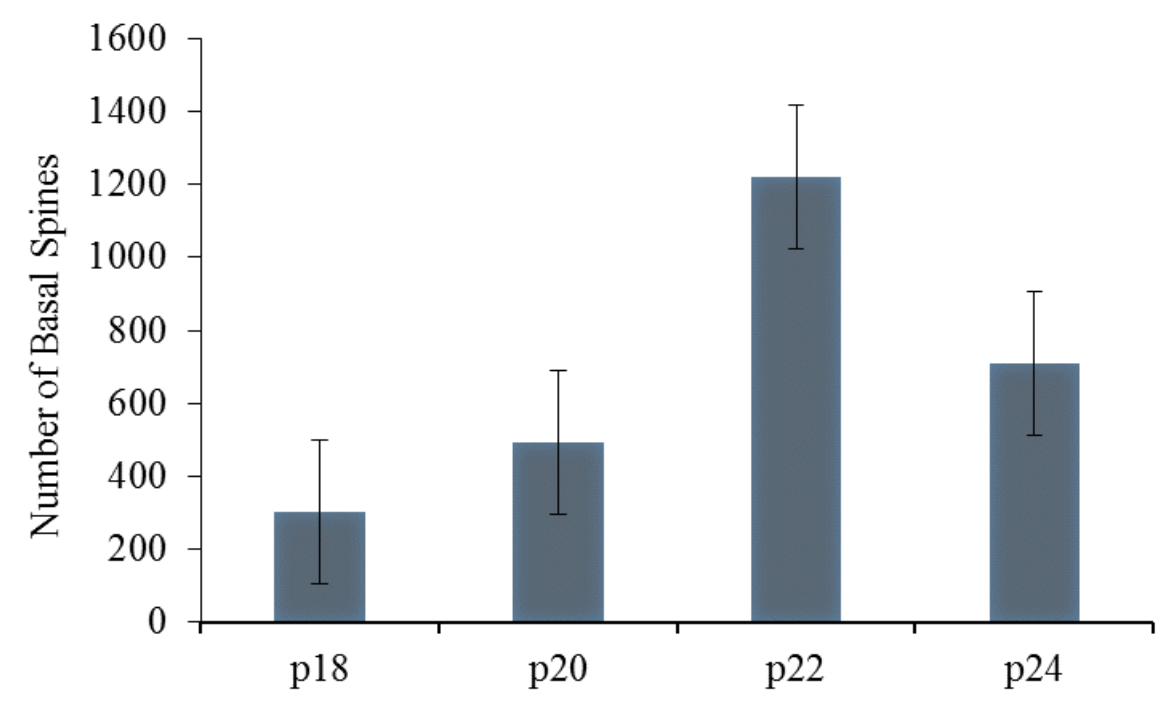

Figure 18. Quantification of spines on the basal dendrites of CA3 pyramidal neurons. No main effect of age was found.

Basal spine density, measured as the number of spines on the basal dendrites divided by length of the basal dendrites of CA3 pyramidal neurons, was obtained and plotted in Figure 19. One-way ANOVA reveled a main effect of age $\left(F_{(3,12)}=4.14, p<0.05\right)$. Tukey post-hoc tests revealed a significant difference between p22 $(m=0.42, s=0.08)$ and p24 $(m=0.20, s=0.11)$ $(p<0.05)$. Basal spine density remained stable at p18 $(m=0.30, s=0.09)$ and p20 $(m=0.32, s=$ $0.04)$. 


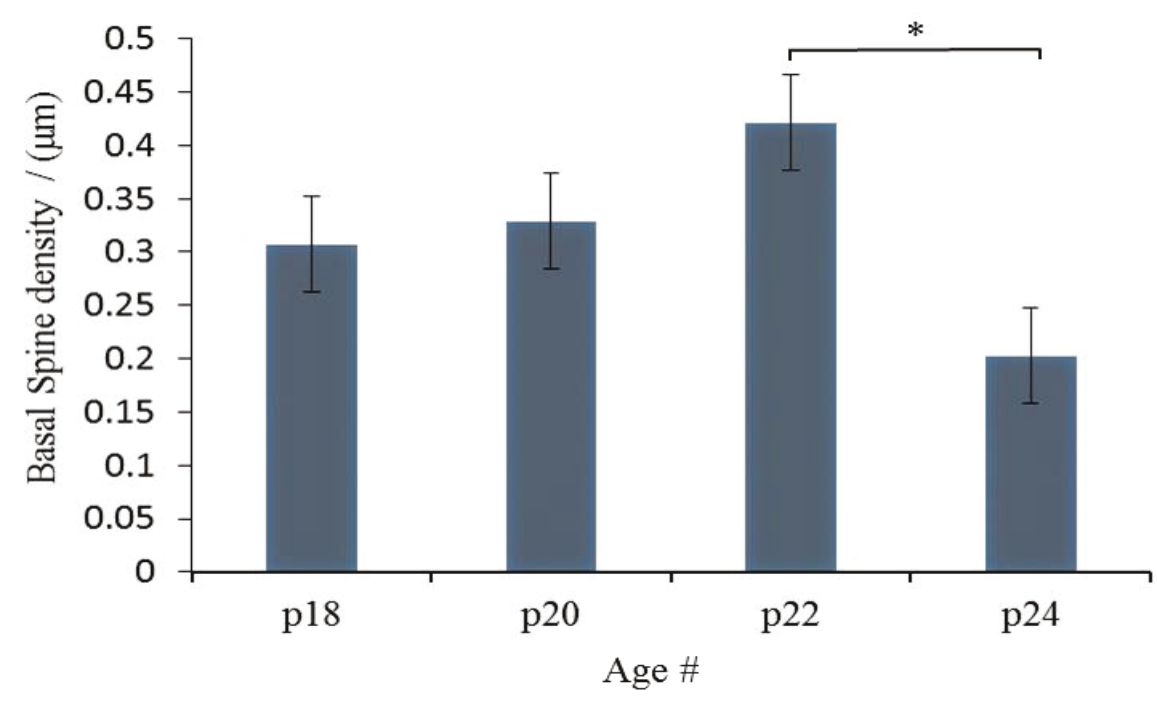

Figure 19. Basal spine density per $\mu \mathrm{m}$ of basal dendrites of the CA3 pyramidal neurons at: p18, p20, p22, and p24. (\#) indicates a main effect of age $(p<0.05) ;(*)$ indicates a significant difference using Tukey post-hoc tests between p22 and p24 $(p<0.05)$.

Basal spine density was obtained by taking the number of spines on the basal dendrites divided by number of basal dendrites (Figure 20). Basal spine density increased slightly from p18 $(m=76.13, s=44.04)$ to $\mathrm{p} 20(m=82.10, s=55.32)$. Then, density increased dramatically at p22 $(m=244.37, s=158.59)$, and then decreased at p24 $(m=215.05, s=163.37)$. Welch ANOVA revealed no statistically significant difference between the age groups $\left(F_{(3,6.13)}=1.8, p\right.$ $=0.244)$. 


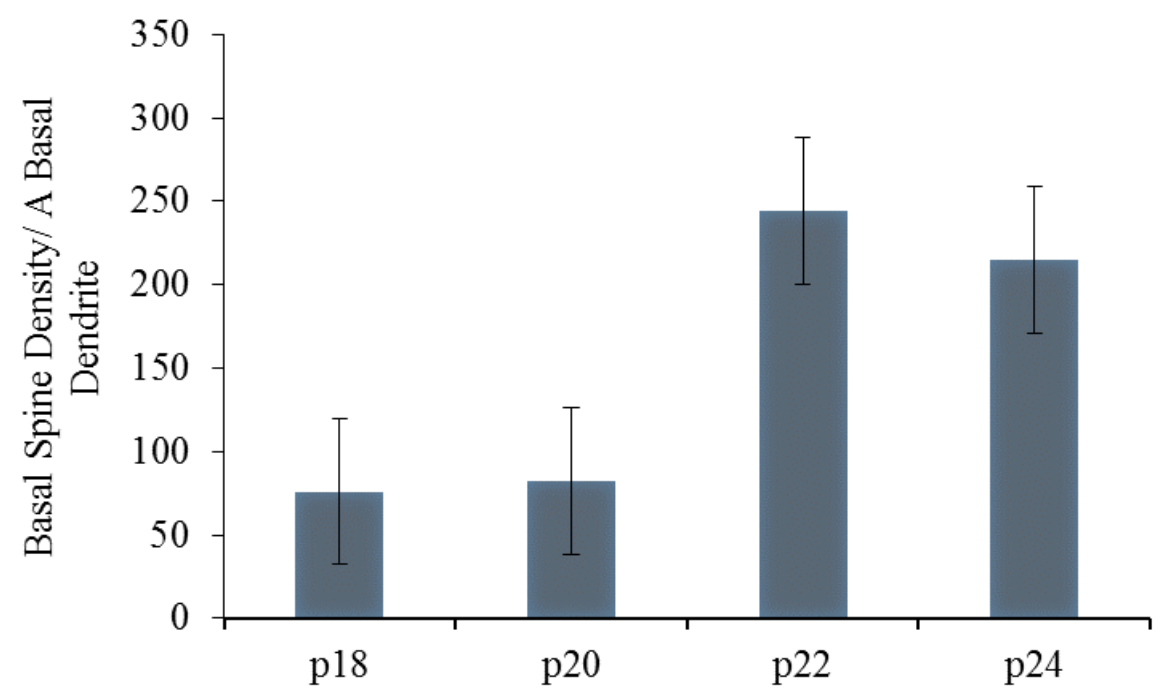

Figure 20. Spine density per a basal dendrite of $\mathrm{CA} 3$ pyramidal neurons was quantified and no main effect of age was found.

\section{Thorny excrescence quantification}

Thorny excrescences (TE) on CA3 neurons from the four age groups (p18, p20, p22 and p24) were quantified from three areas; cell body, apical dendrites and basal dendrites (see Figure 21). Thirty two neurons were included in the analysis ( 8 neurons per age group). Thorny excrescence number increased with age as shown in Figures 20-23 and revealed by statistical analysis. 


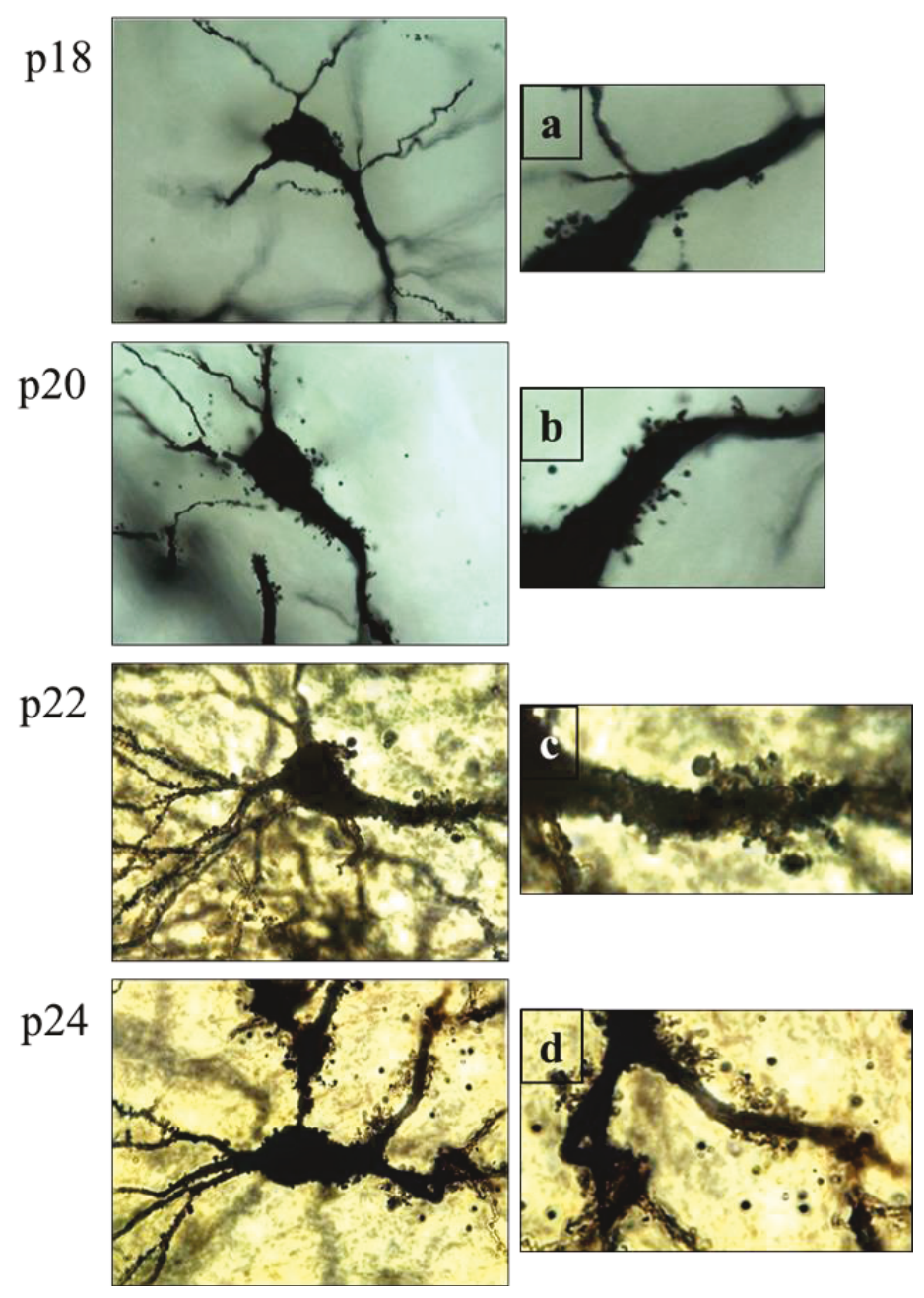

Figure 21. Representative images for Golgi impregnation of TE on CA3 pyramidal neurons at p18, p20, p22 and p24. Images: a, b, c and $\mathbf{d}$ represent TE on the apical dendrites of CA3 neurons, proximal to the cell bodies, at p18, p20, p22 and p24 respectively. Images were taken at $100 \mathrm{X}$.

A Welch ANOVA on the number of TE on the entire neurons (cell body, apical and basal dendrites) revealed a main effect of age, $\left(F_{(3,12.83)}=24.72, p<0.001\right)$. Games-Howell post-hoc tests revealed significant differences between p24 $(m=27, s=16.01)$ and p18 $(m=1.75, s=$ 1.66), $(p<0.05)$ and between $\mathrm{p} 22(m=14.37, s=4.34)$ and $\mathrm{p} 18(p<0.001)$. As Figure 22 shows, there was a significant increase in the number of TE on the entire CA3 neuron with age. 


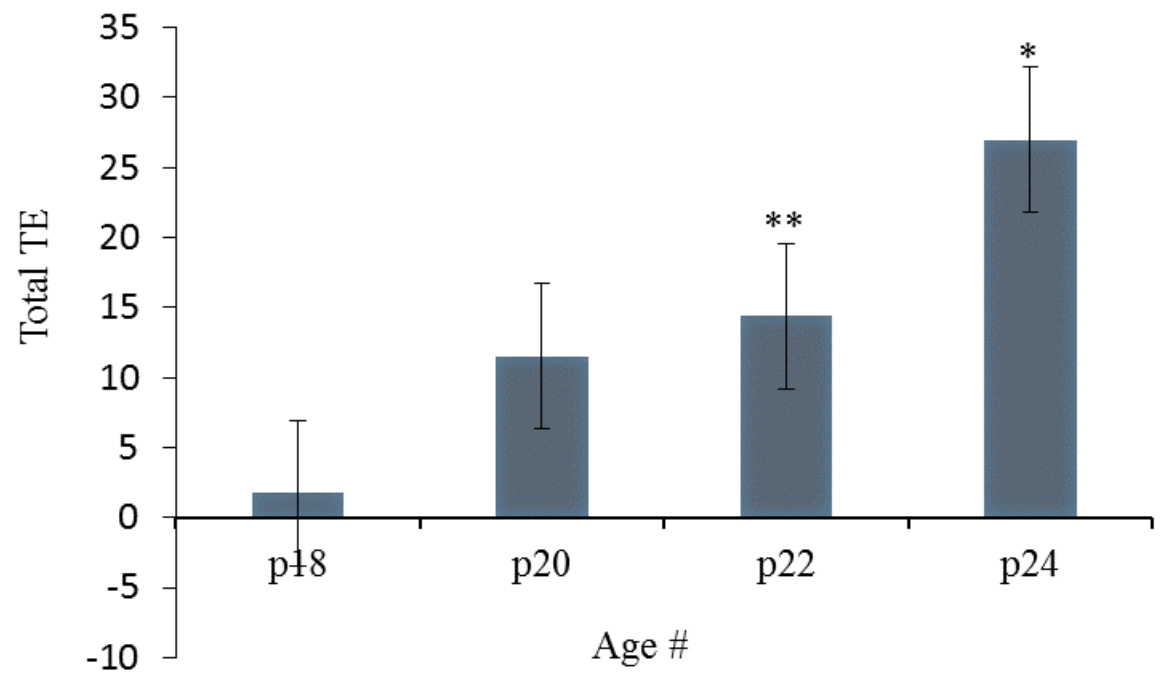

Figure 22. Quantification of TE on the cell body, apical and basal dendrites of CA3

pyramidal neurons at: $\mathrm{p} 18, \mathrm{p} 20, \mathrm{p} 22$, and p24. (\#) indicates a main effect of age $(p<0.001)$; (*) indicates a significant difference using Games-Howell post-hoc tests between p18 and p24 $(p<0.05) ;(* *)$ indicates a significant difference between p18 and p22 $(p<0.001)$.

Welch ANOVA on the number of TE on the apical dendrites revealed a main effect of age, $\left(F_{(3,14.42),} p<0.001\right)$ (Figure 23$)$. Games-Howell post-hoc tests revealed statistically significant differences between p18 $(m=1.12, s=1.72)$ and p22 $(m=6.37, s=1.68)(p<0.001)$ and between $\mathrm{p} 18$ and $\mathrm{p} 24(m=13, s=9.03)(p<0.05)$. 


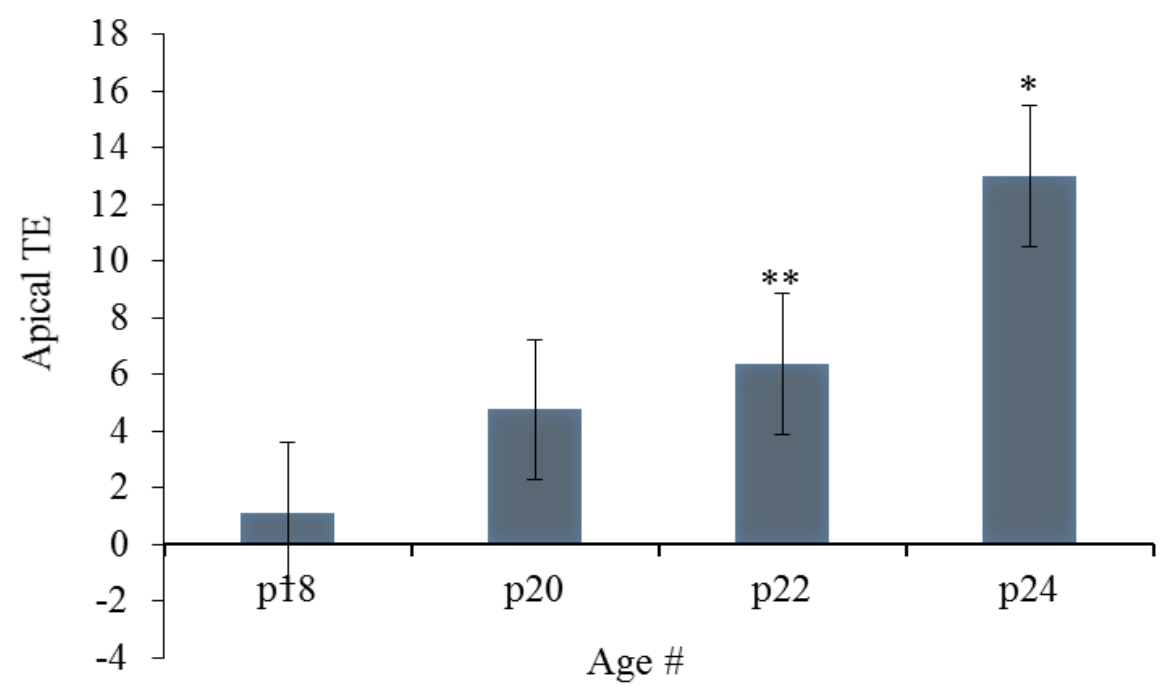

Figure 23. Quantification of TE on the apical dendrites of CA3 pyramidal neurons at: $\mathrm{p} 18$, p20, p22, and p24. (\#) indicates a main effect of age $(p<0.001) ;(*)$ indicates a significant difference using Games-Howell post-hoc tests between p18 and p24 $(p<0.05) ;\left({ }^{* *}\right)$ indicates a significant difference between p18 and p22 $(p<0.001)$.

A one-way ANOVA on the number of TE on basal dendrites of CA3 neurons revealed a main effect of age, $\left(F_{(3,12)}=4.87, p<0.01\right)$. Tukey post-hoc tests revealed a statistically significant difference between p18 $(m=0.37, s=1.06)$ and p24 $(m=9.87, s=7.75)(p<0.01)$. As shown in Figure 24, the means of p20 and p22: $(m=5, s=5.31)$ and $(m=5.75, s=3.15)$ respectively were similar, greater than $\mathrm{p} 18$ and lower than $\mathrm{p} 24$. 


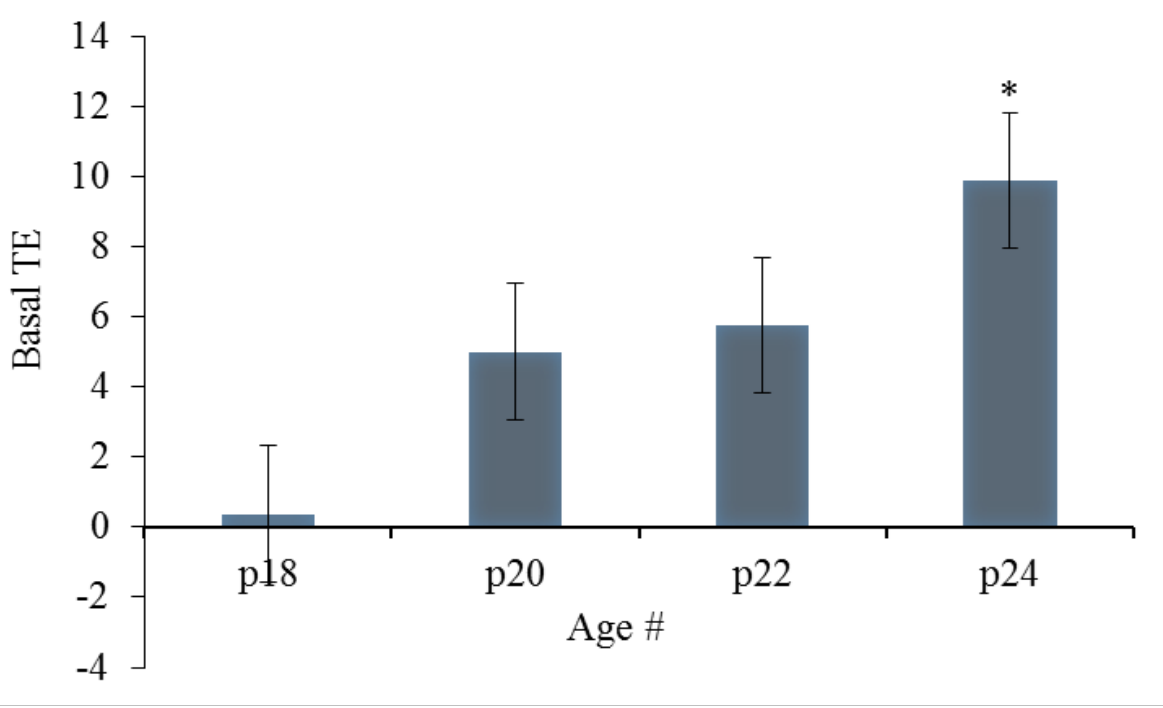

Figure 24. Quantification of TE on basal dendrites of CA3 pyramidal neurons at: p18, p20, p22, and p24. (\#) indicates a main effect of age $(p<0.01)$; $(*)$ indicates a significant difference using Tukey post-hoc tests between p18 and p24 $(p<0.01)$.

A Welch ANOVA on the number of TE on cell bodies of CA3 pyramidal neurons (Figure $25)$ revealed a main effect of age, $\left(F_{(3,14.3)}=25.4, p<0.001\right)$. Games-Howell post-hoc tests revealed statistically significant differences between P18 $(m=0.25, s=0.46)$ and all other age groups: p20 $(m=1.75, s=1.03)(p<0.05), \mathrm{p} 22(m=2.25, s=0.88)(p<0.01)$ and p24 $(m=$ 4.12, $s=1.3)(p<0.001)$. In addition, statistically significant differences were found between p24 and p20 $(p<0.01)$ and between p24 and p22 $(p<0.05)$. 


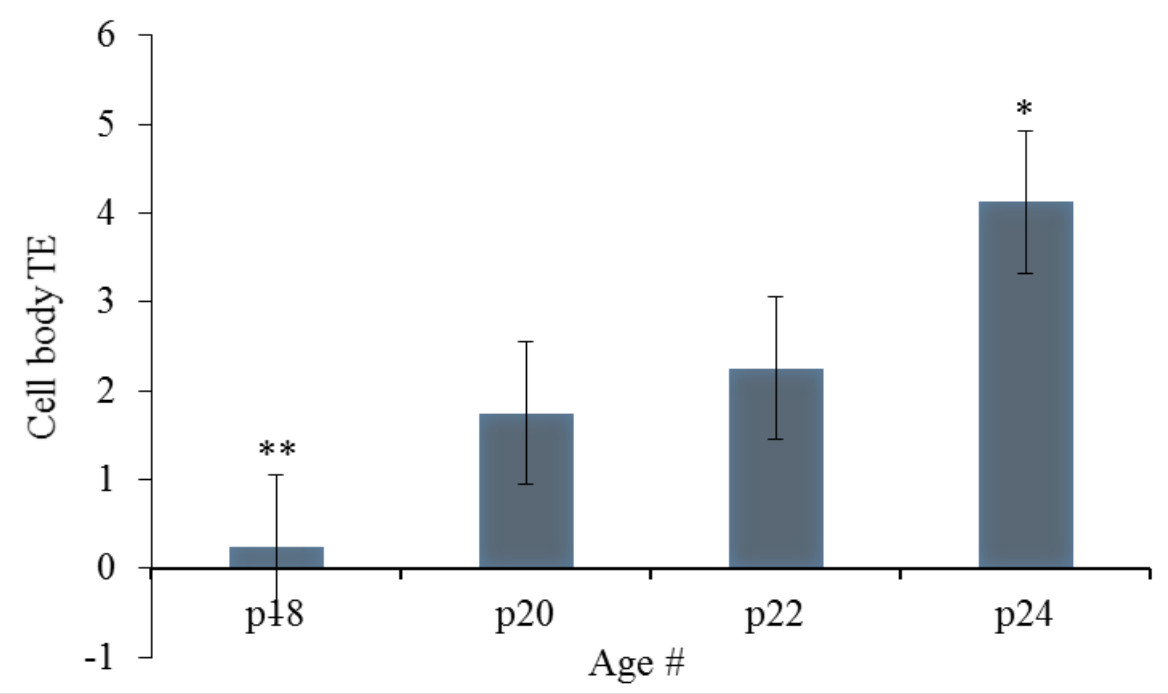

Figure 25. Quantification of TE on cell bodies of CA3 pyramidal neurons at: p18, p20, p22, and p24. (\#) indicates a main effect of age $(p<0.001) ;(*)$ indicates significant differences using Games-Howell post-hoc tests between p24 and p18 $(p<0.001)$, p24 and p20 ( $p<$ $0.01)$, and $\mathrm{p} 24$ and $\mathrm{p} 22(p<0.05) ;(* *)$ indicates significant differences between $\mathrm{p} 18$ and $\mathrm{p} 20(p<0.05)$ and $\mathrm{p} 18$ and $\mathrm{p} 22(p<0.01)$.

\section{Experiment 2}

Juvenile rats were injected with different doses of the AMPA receptor antagonist NBQX $(0 \mathrm{mg} / \mathrm{kg}, 5 \mathrm{mg} / \mathrm{kg}$ or $10 \mathrm{mg} / \mathrm{kg})$ every day before they were trained on the Morris water maze. The training phase consisted of three days when the rats were $\mathrm{p} 18, \mathrm{p} 19$ and $\mathrm{p} 20$. There were eight trials per day and each trial lasted for a maximum of 60 seconds. The platform was hidden under the surface of the water in the same location throughout all the trials. 


\section{Behavioral results}

Results from the training phase: $\mathrm{p} 18, \mathrm{p} 19$ and $\mathrm{p} 20$ :

Latency, pathlength and thigmotaxis were captured and quantified using a video camera and tracking system (HVS Image 2010). Latency, in seconds, is the time required to find or reach the hidden platform after a rat enters the pool. Thigmotaxis is "the tendency to swim close to or follow the wall around the perimeter of the (pool) tank" (Vorhees \& Williams, 2006). A greater measurement on latency, pathlength or thigmotaxis indicates that the rat did not learn or remember the location of the platform. A two-way mixed ANOVA (a between-subjects factorial ANOVA and a within-subjects repeated measures ANOVA) was used to determine if there was an interaction between NBQX dose and days of training on the three measurements.

\section{Latency:}

Latencies were averaged over the eight trials to obtain a daily average for each group: 0mg, $5 \mathrm{mg}$ and 10mg. Data are plotted in Figure 26. Tests of between subjects effects, mixed two-way ANOVA, revealed a significant interaction between NBQX dose and training day $\left(F_{(4,30)}=5.86, p<0.01\right.$, partial $\left.\eta^{2}=0.439\right)$.

A two-way factorial (between subjects) ANOVA using the latency as the dependent variable and days of training and NBQX dose as independent variables was used to test the main effect of dose on each day. On the first day (p18), there was no statistically significant difference in latencies between the three groups $\left(F_{(2,15)}=1.68, p=0.219\right.$, partial $\left.\eta^{2}=0.183\right)$. There was a statistically significant difference in latencies between the NBQX groups on day $2(\mathrm{p} 19)\left(F_{(2,15)}=\right.$ $5.07, p<0.05$, partial $\left.\eta^{2}=0.403\right)$, and on day $3(\mathrm{p} 20)\left(F_{(2,15)}=15.28, p<0.001\right.$, partial $\eta^{2}=$ $0.671)$ 
Post-hoc Tukey tests were used for pairwise multiple comparisons on days 2 and 3. On day 2, latency was significantly longer in the $5 \mathrm{mg}$ group $(\mathrm{M}=15.13, \mathrm{SE}=5.76, p<0.05)$ and $10 \mathrm{mg}(\mathrm{M}=16.55, \mathrm{SE}=5.76, p<0.05)$ compared to the $0 \mathrm{mg}$ group. Latency in the 5mg group was not significantly different than the $10 \mathrm{mg}$ group $(\mathrm{M}=1.4, \mathrm{SE}=5.76, p=0.967)$.

Post-hoc Tukey tests on day 3 revealed significantly longer latencies in the $5 \mathrm{mg}$ group $(\mathrm{M}=22.61, \mathrm{SE}=5.31, p<0.01)$ and $10 \mathrm{mg}$ group $(\mathrm{M}=27.56, \mathrm{SE}=5.31, p<0.001)$ compared to the $0 \mathrm{mg}$ group. Latencies in the $5 \mathrm{mg}$ group were not significantly different than the $10 \mathrm{mg}$ group $(\mathrm{M}=4.95, \mathrm{SE}=5.31, p=0.630)$.

A two-way repeated measures ANOVA was conducted to investigate the latency change in each NBQX dose group over time (days of training). There was a significant effect of day on the latencies in the 0mg group, $\left(F_{(2,10)}=14.52, p<0.01\right.$. partial $\left.\eta^{2}=0.744\right)$ and the $5 \mathrm{mg}$ group $\left(F_{(2,10)}=5.13, p<0.05\right.$, partial $\left.\eta^{2}=0.506\right)$. There was no significant effect of time on the latency in the $10 \mathrm{mg}$ group.

Bonferroni-adjusted Pairwise comparison for the 0mg group revealed no significant difference in latencies between days 1 and day 2 of training $(\mathrm{M}=15.93, \mathrm{SE}=4.75, p=0.061)$, but latencies were significantly reduced on day 3 of training $(\mathrm{M}=27.63, \mathrm{SE}=5.84, p<.05)$. Bonferroni-adjusted pairwise comparisons revealed no significant differences in latencies for the 5 and 10mg NBQX groups between all days of training. 


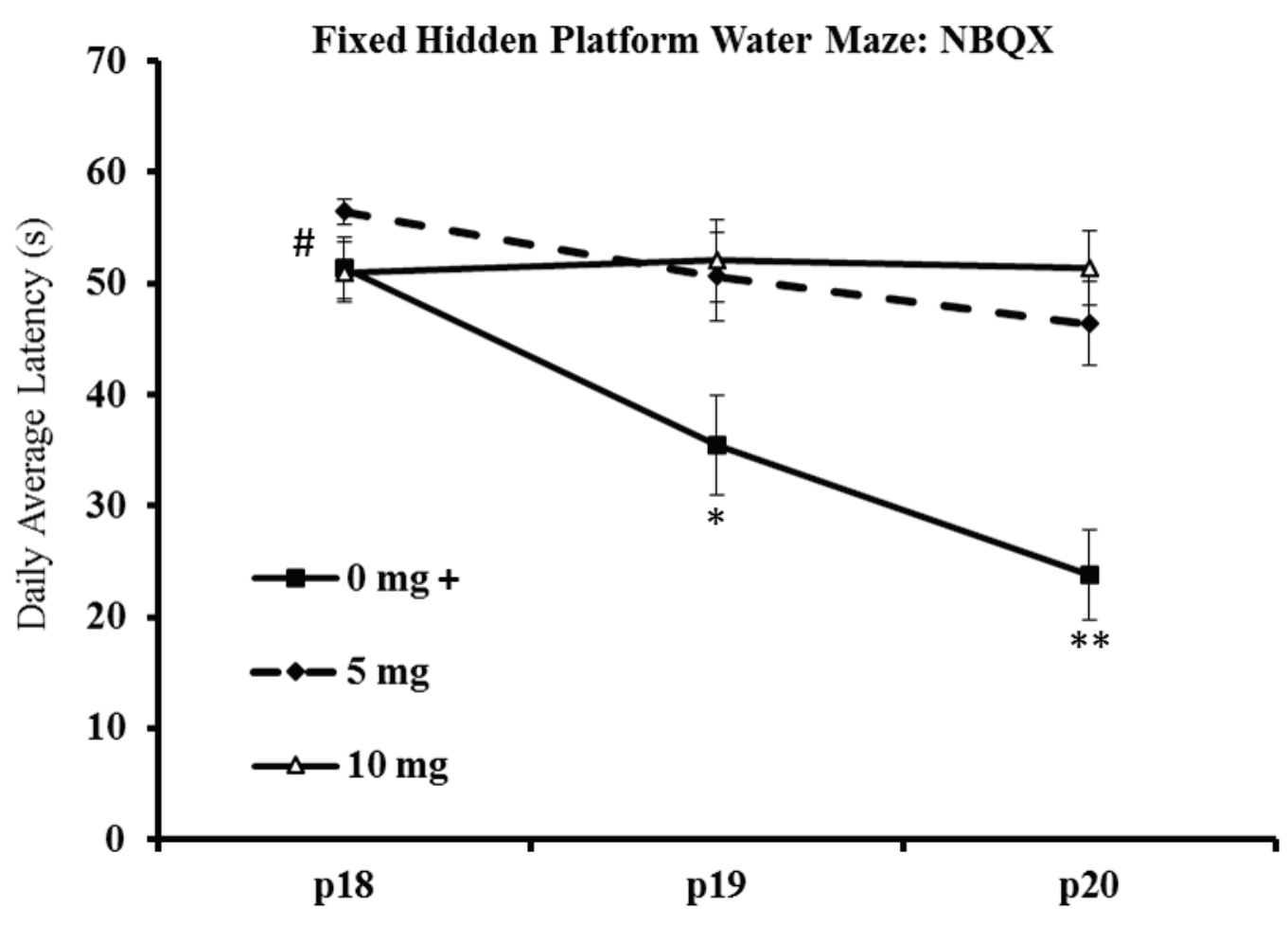

Figure 26. Latency to reach the hidden platform averaged across eight trials of daily training from p18 to p20. (\#) indicates a significant interaction between days of training and NBQX doses $(p<0.01) .(*)$ indicates a statistically significant reduction in latency in the $0 \mathrm{mg}$ groups compared to the $5 \mathrm{mg}$ group $(p<0.05)$ and the10mg group $(p<0.05)$ on the second day of training (p19). $\left({ }^{* *}\right)$ indicates a significant reduction in latency in the $0 \mathrm{mg}$ group compared to the $5 \mathrm{mg}$ group $(p<0.01)$ and the $10 \mathrm{mg}$ groups $(p<0.001) .(+)$ indicates a significant reduction in latency in the $0 \mathrm{mg}$ group over days of training $(p<0.05)$.

\section{Pathlength:}

Pathlength was averaged over the eight trials to obtain a daily average pathlength for each group. Data are plotted in Figure 27. Two-way ANOVA revealed a significant interaction between NBQX dose and day of training $\left(F_{(4,30)}=6.8, p<0.01\right.$, partial $\left.\eta^{2}=0.476\right)$. 
A two-way factorial (between subjects) ANOVA, using pathlength as the dependent variable and days of training and NBQX dose as independent variables, was used to test the main effect of dose in each day. There was a significant difference in pathlength between the three groups on all three days of training: $18\left(F_{(2,15)}=7.09, p<0.01\right.$, partial $\left.\eta^{2}=0.486\right), \mathrm{p} 19\left(F_{(2,15)}=\right.$ 4.86, $p<0.05$, partial $\left.\eta^{2}=0.393\right)$, and $\mathrm{p} 20\left(F_{(2,15)}=9.25, p<0.01\right.$, partial $\left.\eta^{2}=0.552\right)$.

Post-hoc Tukey tests were used to determine the main effect of dose on each day of training. On day 1 (p18), pathlength was significantly longer in the $5 \mathrm{mg}$ group compared to the $10 \mathrm{mg}$ group $(\mathrm{M}=2.39, \mathrm{SE}=.65, p<0.01)$. The $0 \mathrm{mg}$ group was not significantly shorter than $5 \mathrm{mg}$ group $(\mathrm{M}=0.77, \mathrm{SE}=0.64, p=0.445)$ nor the $10 \mathrm{mg}$ group $(\mathrm{M}=1.61, \mathrm{SE}=0.64, p=$ $0.061)$.

Post-hoc Tukey tests on day 2 (p19) revealed significantly longer pathlength in the 5mg group $(\mathrm{M}=2.73, \mathrm{SE}=1.04, p<0.05)$ and the $10 \mathrm{mg}$ group $(\mathrm{M}=2.86, \mathrm{SE}=1.04, p<0.05)$ compared to the $0 \mathrm{mg}$ group. Pathlength in $5 \mathrm{mg}$ group was not significantly shorter than the $10 \mathrm{mg}$ group $(\mathrm{M}=0.132, \mathrm{SE}=1.03, p=0.991)$.

Post-hoc Tukey tests on day 3 (p20) of training revealed significantly longer pathlength in the $5 \mathrm{mg}(\mathrm{M}=5.18, \mathrm{SE}=1.49, p<0.01)$ and $10 \mathrm{mg}(\mathrm{M}=5.91, \mathrm{SE}=1.49, p<0.01)$ groups compared to the $0 \mathrm{mg}$ group. Pathlength in the $5 \mathrm{mg}$ group was not significantly shorter than the $10 \mathrm{mg}$ group $(\mathrm{M}=.731, \mathrm{SE}=1.49, p=0.878)$.

A two-way repeated measures ANOVA was conducted to test the changes in the pathlength in each NBQX dose group over time. There was a significant effect of time (day of training) on the pathlength in the $0 \mathrm{mg}$ group $\left(F_{(2,10)}=5.13, p<0.05\right.$. partial $\left.\eta^{2}=0.507\right)$ and in 
the 10mg group $\left(F_{(2,10)}=8.34, p<0.01\right.$, partial $\left.\eta^{2}=0.625\right)$. There was no significant effect of time on the pathlength in the $5 \mathrm{mg}$ group $\left(F_{(2,10)}=0.109, p=0.898\right.$, partial $\left.\eta^{2}=0.021\right)$.

Bonferroni-adjusted pairwise comparisons revealed a statistically significant increase in pathlength in the $10 \mathrm{mg}$ group on day 2 compared to day 1 of training $(\mathrm{M}=2.49, \mathrm{SE}=0.53, p<$ $0.05)$, and a nearly significant increase in day 3 compared to day $1(\mathrm{M}=3.48, \mathrm{SE}=1.04, p=$ 0.062). For the $0 \mathrm{mg}$ and $5 \mathrm{mg}$ groups, pairwise comparisons revealed no statistically significant difference over days of training.

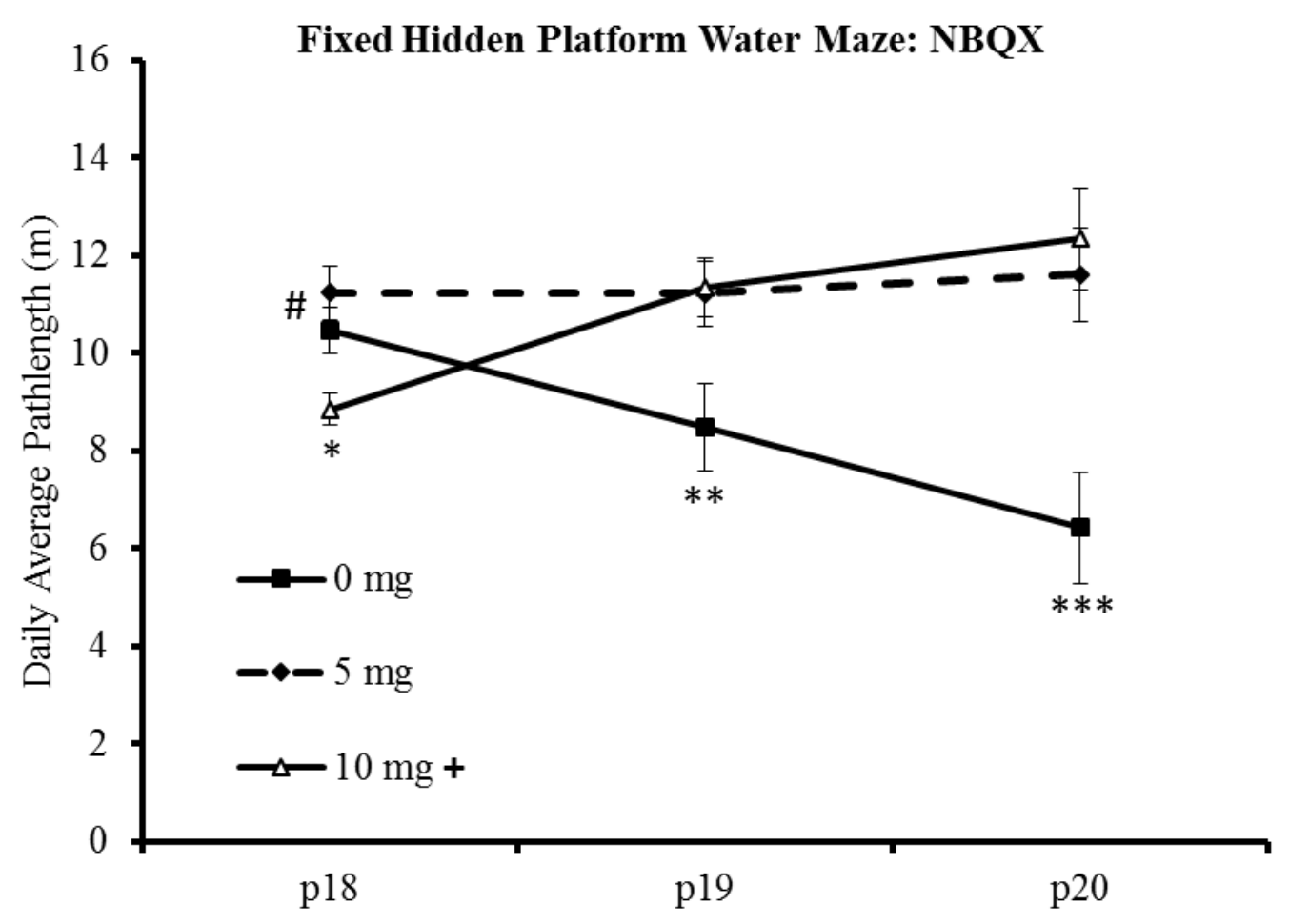

Figure 27. Pathlength averaged across eight trials of daily training from p18 to p20. (\#) indicates a significant interaction found between day of training and NBQX dose $(p<0.01)$.

$\left.{ }^{*}\right)$ indicates a statistically significant reduction in the $10 \mathrm{mg}$ group compared to $5 \mathrm{mg}$ group 
$(p<0.05)$ in the first day of training $\mathrm{p} 18 .(* *)$ indicates a significant reduction for the $0 \mathrm{mg}$ group compared to $5 \mathrm{mg}(p<0.05)$ and $10 \mathrm{mg}$ groups $(p<0.05)$ on day $2(\mathrm{p} 19) .(* * *)$ indicates a significant reduction for the $0 \mathrm{mg}$ group compared to $5 \mathrm{mg}(p<0.01)$ and $10 \mathrm{mg}$ groups $(p<0.01)$ on day $3(\mathrm{p} 20)$. (+) Indicate a significant increase in pathlength for the $10 \mathrm{mg}$ group over days of training $(p<0.05)$.

\section{Thigmotaxis:}

Thigmotaxis was averaged over the eight trials to obtain a daily average for each group (Figure 28). Two-way ANOVA revealed a significant interaction between NBQX dose and day of training $\left(F_{(4,30)}=4.98, p<0.01\right.$, partial $\left.\eta^{2}=0.399\right)$.

A two-way factorial (between-subjects) ANOVA, using thigmotaxis as the dependent variable and days of training and NBQX dose as independent variables, was used to test the main effect of dose in each day. There were significant differences in thigmotaxis between the three groups on day $2\left(F_{(2,15)}=7.08, p<0.01\right.$, partial $\left.\eta^{2}=0.486\right)$, and day $3\left(F_{(2,15)}=13.86, p<0.001\right.$, partial $\left.\eta^{2}=0.649\right)$.

Post-hoc Tukey tests were used to test the main effects on day 2 and 3. Pairwise multiple comparisons on day 2 revealed a significant increase in thigmotaxis in the 10mg group compared to the $0 \mathrm{mg}$ group $(\mathrm{M}=28.99, \mathrm{SE}=7.72, p<0.01)$. The $5 \mathrm{mg}$ group was not significantly greater than the $0 \mathrm{mg}$ group $(\mathrm{M}=16.16, \mathrm{SE}=7.72, p=0.125)$ nor significantly lower than the $10 \mathrm{mg}$ group $(\mathrm{M}=12.83, \mathrm{SE}=7.72, p=0.251)$.

Post-hoc Tukey tests on day 3 revealed significantly greater thigmotaxis in the $5 \mathrm{mg}$ group $(\mathrm{M}=31.43, \mathrm{SE}=8.29, p<0.01)$, and 10mg group $(\mathrm{M}=41.96, \mathrm{SE}=8.29, p<0.001)$ compared to the 0mg group. 
A two-way repeated measures ANOVA was conducted to investigate the changes in thigmotaxis in each NBQX dose group over time. There was a significant effect of time (day of training) on thigmotaxis in the 0mg group, $\left(F_{(2,10)}=12.51, p<0.01\right.$. partial $\left.\eta^{2}=0.715\right)$, but no significant differences for 5mg and 10mg groups were found. Bonferroni-adjusted Pairwise comparisons on $0 \mathrm{mg}$ group revealed a significant reduction in thigmotaxis on day 3 compared to day $1(\mathrm{M}=23.44, \mathrm{SE}=4.08, p<0.01)$, and day $2(\mathrm{M}=13.48, \mathrm{SE}=3.24, p<0.05)$. Thigmotaxis was not statistically significantly lower in day 2 when compared with day $1(\mathrm{M}=9.82, \mathrm{SE}=6.26$, $p=0.533)$.

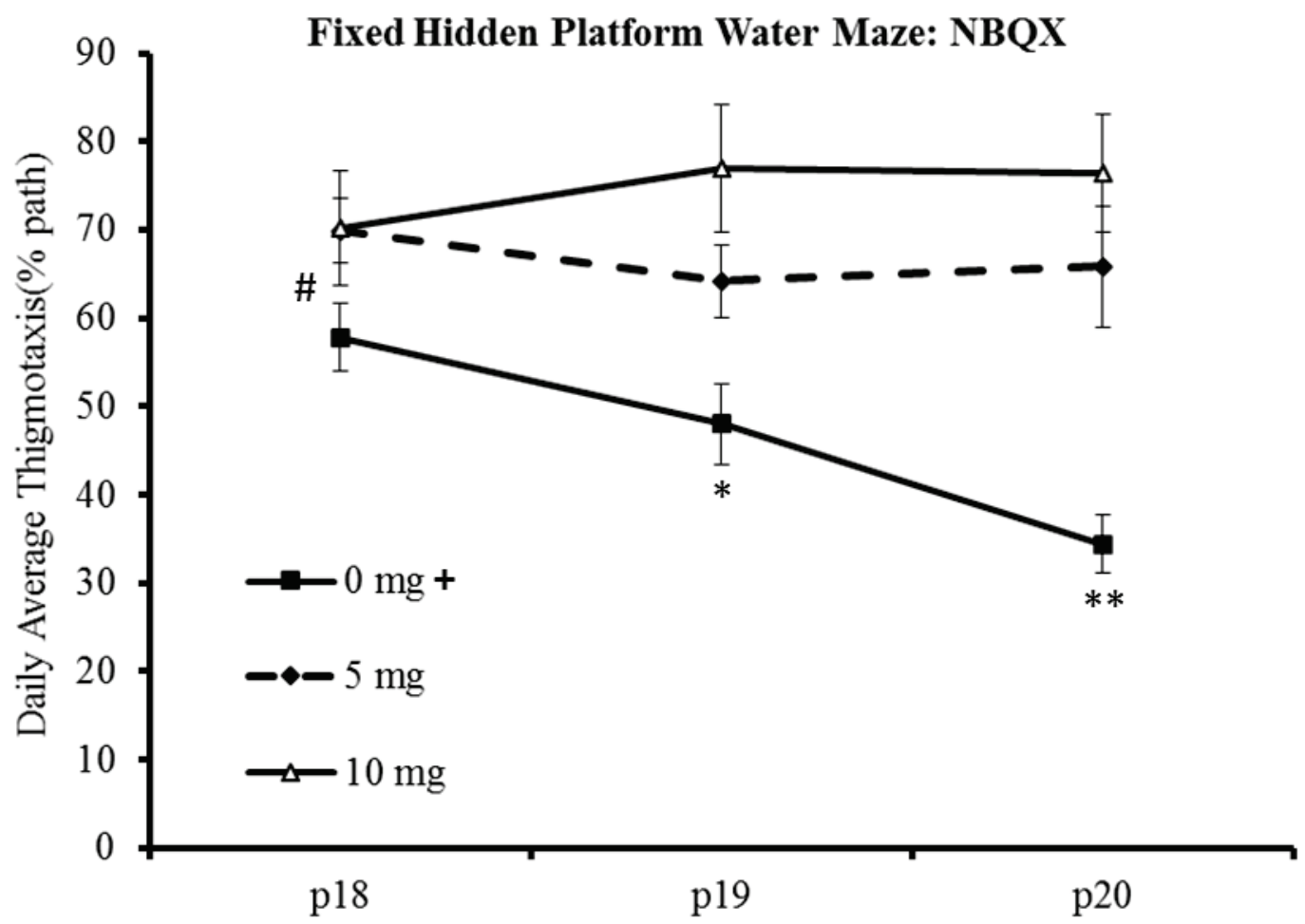

Figure 28. Thigmotaxis averaged across eight trials of daily training from p18 to p20. (\#) indicates a significant interaction between day of training and NBQX dose $(p<0.01) .\left(^{*}\right)$ indicates a statistically significant reduction in the $0 \mathrm{mg}$ group compared to $10 \mathrm{mg}$ group $(p<$ $0.01)$ on day 2 of training (p19). (**) indicates a significant reduction in the $0 \mathrm{mg}$ group 
compared to $5 \mathrm{mg}(p<0.01)$ and $10 \mathrm{mg}$ groups $(p<0.001)$ on day $3(\mathrm{p} 20) .(+)$ indicates a significant decrease in thigmotaxis in the $0 \mathrm{mg}$ group over days of training $(p<0.01)$.

Results from the probe test:

The test was one day when the rats were 41 days old ( 3 weeks after the last day of training). The probe test included a single trial that lasted for 60 seconds. The platform was removed from the pool. The time every rat spent in the quadrant where the platform was located (target) was measured along with the time spent in the opposite quadrant (opposite). Three t-tests were separately performed for each group to compare the time rats spent in each quadrant: (0mg: opposite vs. target), (5mg: opposite vs. target) and (10mg: opposite vs. target). Data are plotted in Figure 29.

A $t$-test on the $0 \mathrm{mg}$ group revealed significantly more time spent in the target quadrant ( $\mathrm{m}$ $=43.55, s=15.53)$ than the opposite quadrant $(m=19.30, s=15.81),(t(5)=1.94, p \leq 0.05)$. A $t$ test on the $5 \mathrm{mg}$ group revealed more time spent in the target quadrant $(m=40.40, s=15.10)$ than the opposite quadrant $(m=15.71, s=8.83),(t(5)=2.737, p<0.05)$. For the10mg group, no difference was found between the times spent in the target and opposite quadrant: target $(m=$ $24.68, s=5.05)$ and opposite $(m=30.28, s=9.36)(t(5)=-1.202, p=0.256)$. 


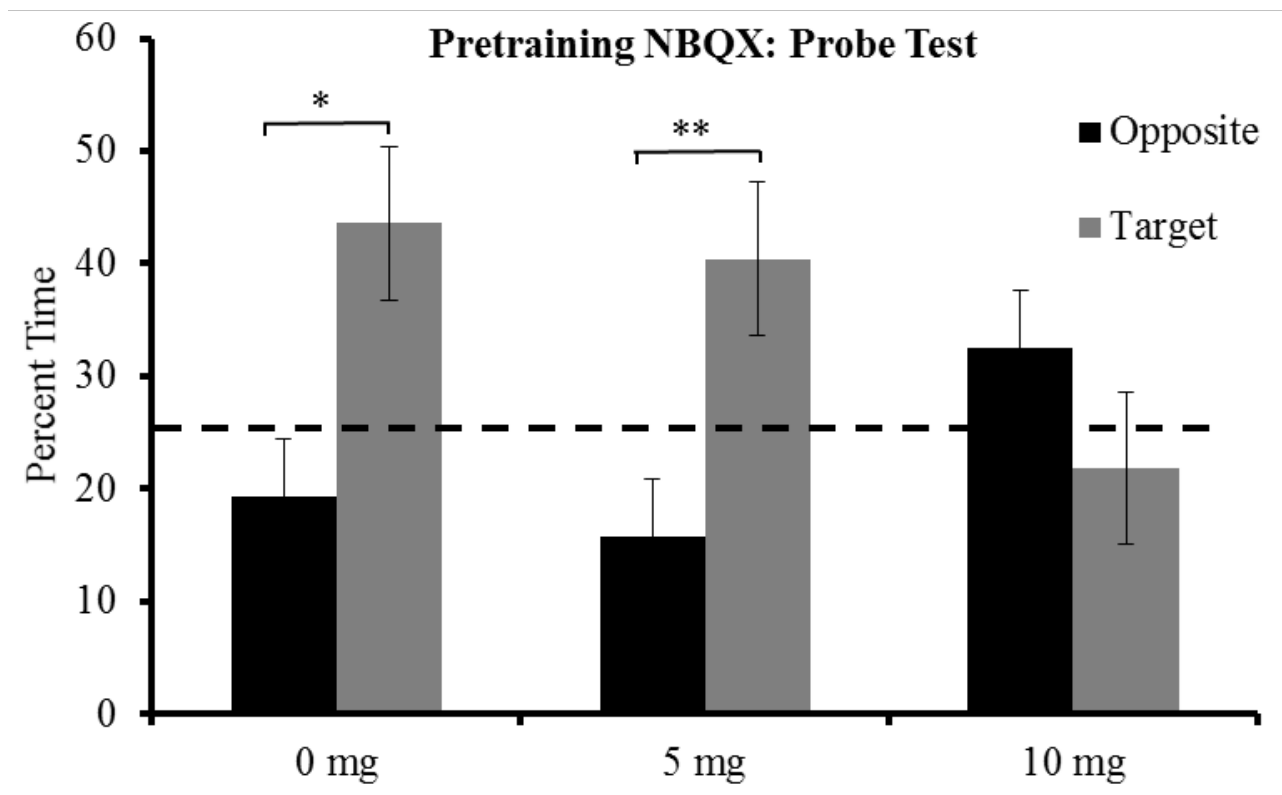

Figure 29. Averaged percentage of time rats spent in the target vs. the opposite quadrant in the three dose groups: $0 \mathrm{mg}, 5 \mathrm{mg}$ and $10 \mathrm{mg}$. (*) indicates a significant difference between time spent in the target compared to the opposite quadrant in the group $0 \mathrm{mg}(p<0.05) .(* *)$ indicates a significant difference between time spent in the target quadrant compared to the opposite quadrant in the group $5 \mathrm{mg}(p<0.05)$.

\section{CA3 structural analysis:}

Following the testing phase, 4 rats from each group (0mg, 5mg and $10 \mathrm{mg})$ were euthanized and brains were obtained for Golgi analysis (see Figure 30). Four CA3 pyramidal neurons per group were reconstructed and analyzed following the same procedures as described for Experiment 1. 


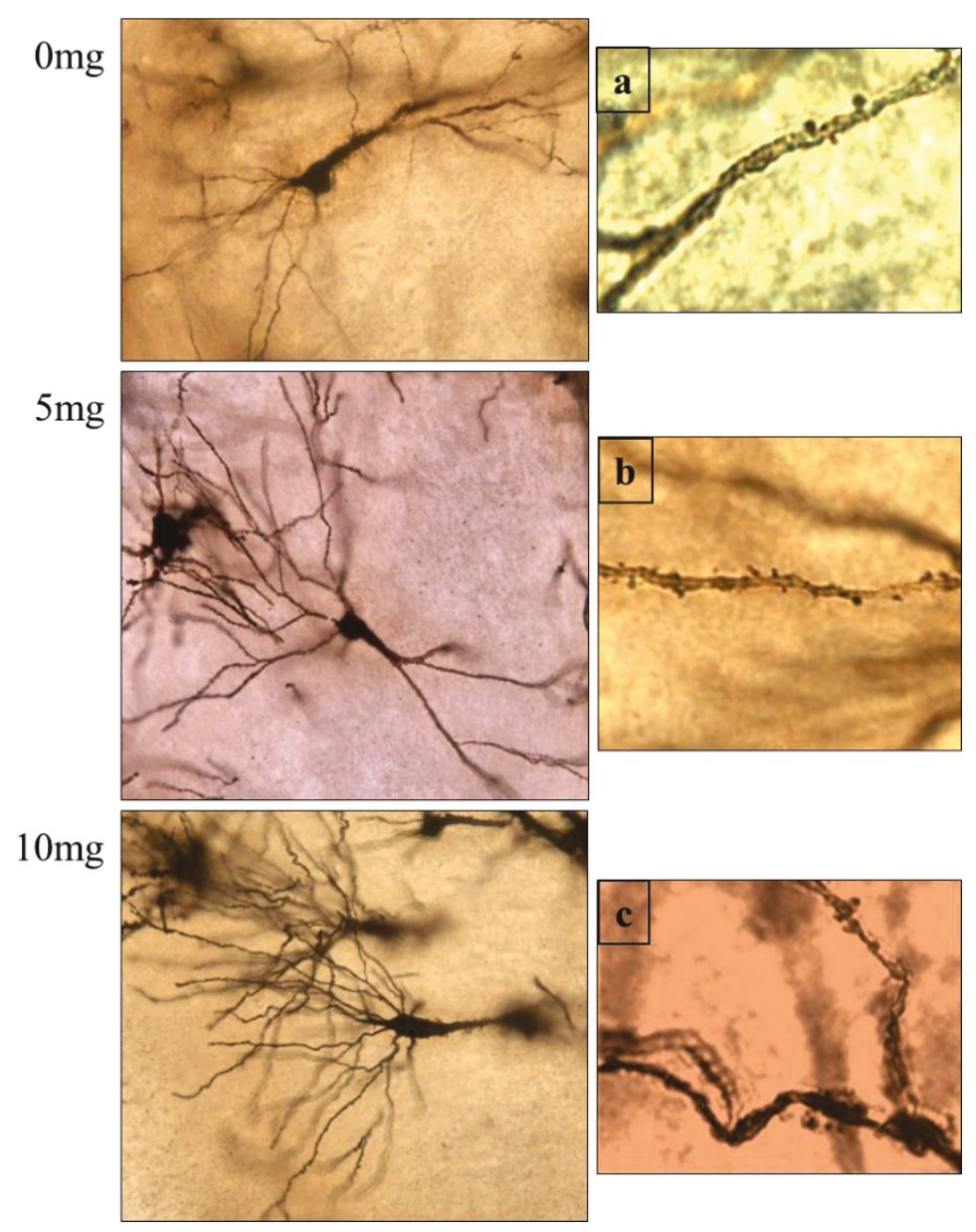

Figure 30. Representative images for Golgi impregnation of CA3 pyramidal neurons in groups: 0mg, 5mg and 10mg taken at 20X. Images: a, b and $\mathbf{c}$ represent spine densities on the basal dendrites of CA3 neurons in groups $0 \mathrm{mg}, 5 \mathrm{mg}$ and $10 \mathrm{mg}$ respectively. Images were taken at $100 \mathrm{X}$.

\section{$\underline{\text { Cell body area }\left(\mu \mathrm{m}^{2}\right)}$}

A one-way ANOVA on the cell body area revealed no main effect of dose $\left(F_{(2,9)}=1.36, p\right.$ $=0.303)$. Averages of the cell bodies, as shown in Figure 31, were similar in the 0mg group $(m=$ 343.33, $s=45.28)$ and 5mg group $(m=321.68, s=75.31)$ and slightly greater in the 10mg group $(m=395.85, s=71.02)$. 


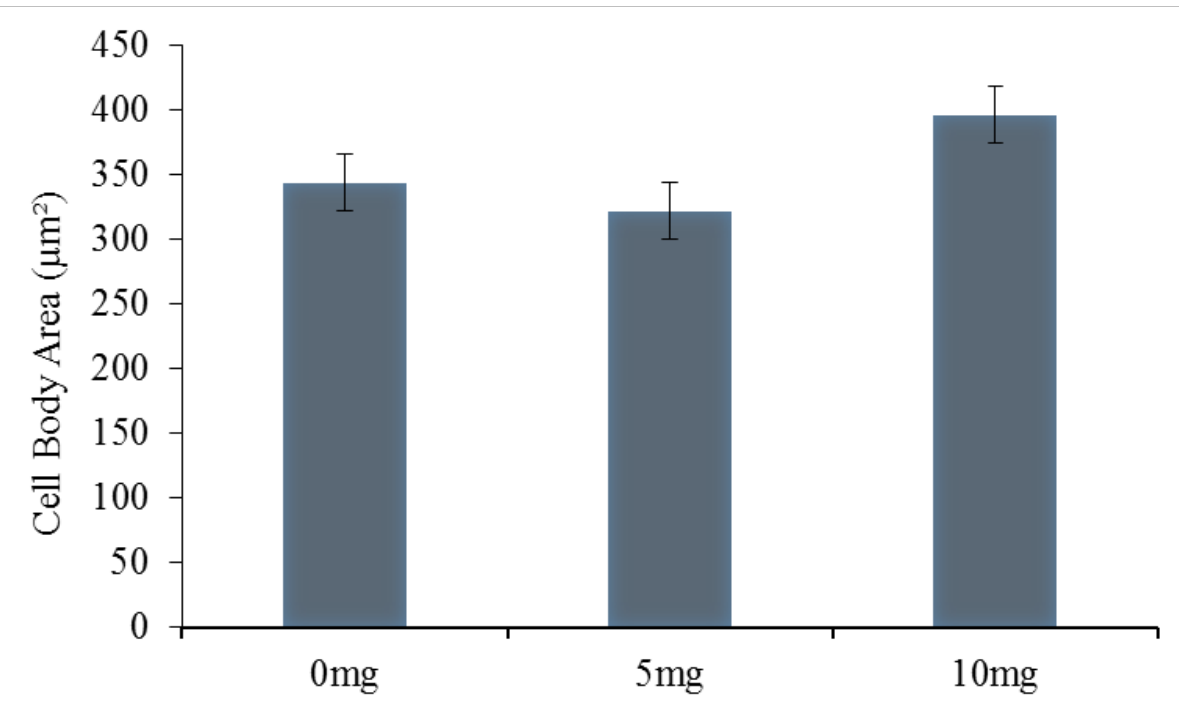

Figure 31. Areas of the cell bodies of CA3 pyramidal neurons after injections with different doses of NBQX and training on the MWM. No main effect of dose was found.

\section{Number of dendrites}

A one-way ANOVA on the number of basal dendrites for each group, plotted in Figure 32 , revealed no main effect of does $\left(F_{(2,9)}=1.84, p=0.213\right)$. Basal dendrite number was lowest in the $0 \mathrm{mg}$ group $(m=4.25, s=0.95)$, then slightly increased in the $5 \mathrm{mg}$ group to $(m=6, s=$ $1.82)$, and in the 10mg group $(m=5.75, s=1.25)$. 


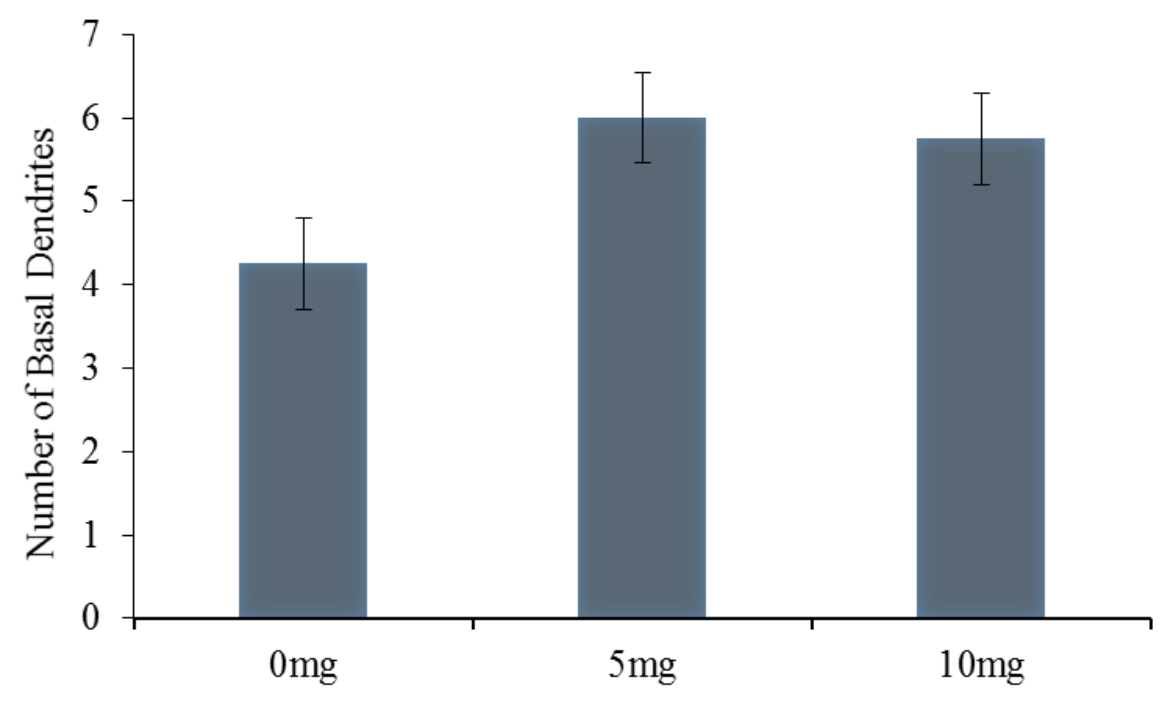

Figure 32. Quantification of basal dendrites of $\mathrm{CA} 3$ pyramidal neurons after injections with different doses of NBQX and training on the MWM. No main effect of dose was found.

Number of apical dendrites, plotted in Figure 33, increased with dose from 0mg $(m=1, s$ $=0), 5 \mathrm{mg}(m=1.25, s=0.5)$, to $10 \mathrm{mg}(m=1.5, s=0.57)$. However, one-way ANOVA revealed no significant difference between groups $\left(F_{(2,9)}=1.28, p=0.323\right)$. 


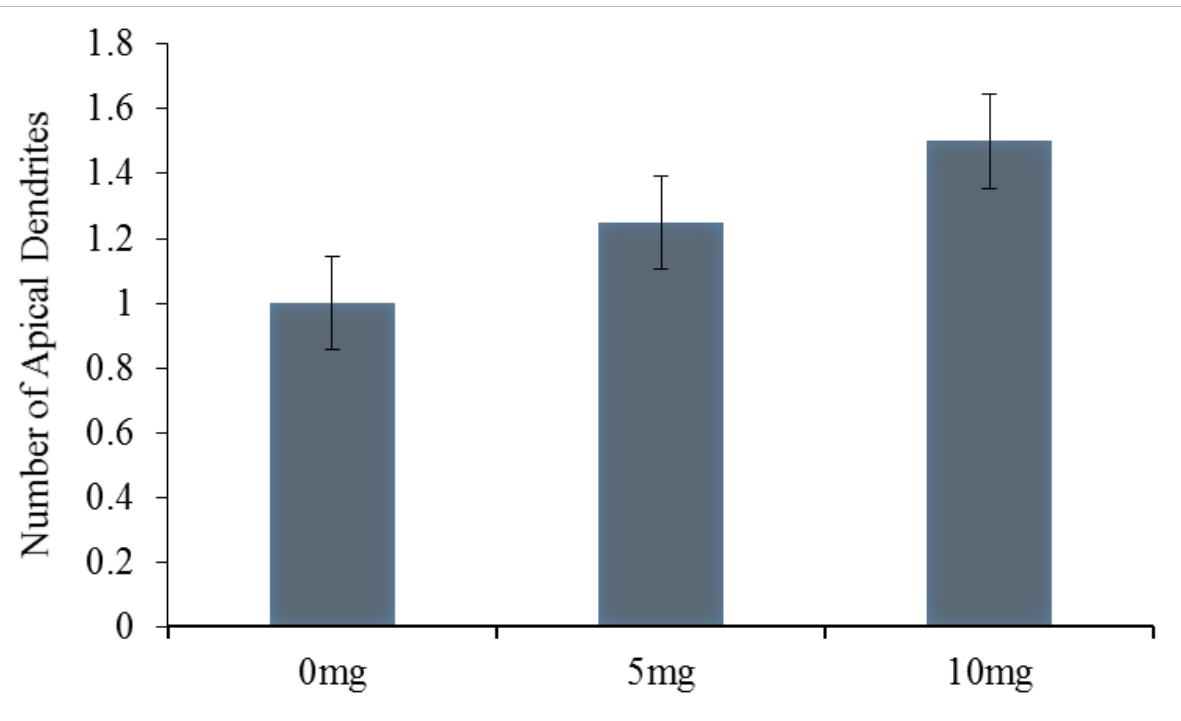

Figure 33. Quantification of the apical dendrites of CA3 pyramidal neurons after injections with different doses of NBQX and training on the MWM. No main effect of dose was found.

\section{$\underline{\text { Branch nodes and ends }}$}

Number of nodes in the apical and basal dendrites, plotted in Figure 34, increased in the $5 \mathrm{mg}$ group $(m=30.5, s=2.88)$ compared to $0 \mathrm{mg}$ group $(m=27.75, s=15.43)$ with a slight decrease in the 10mg group $(m=30, s=7.78)$. No statistically significant difference between groups was found using one-way ANOVA $\left(F_{(2,9)}=0.084, p=0.920\right)$. 


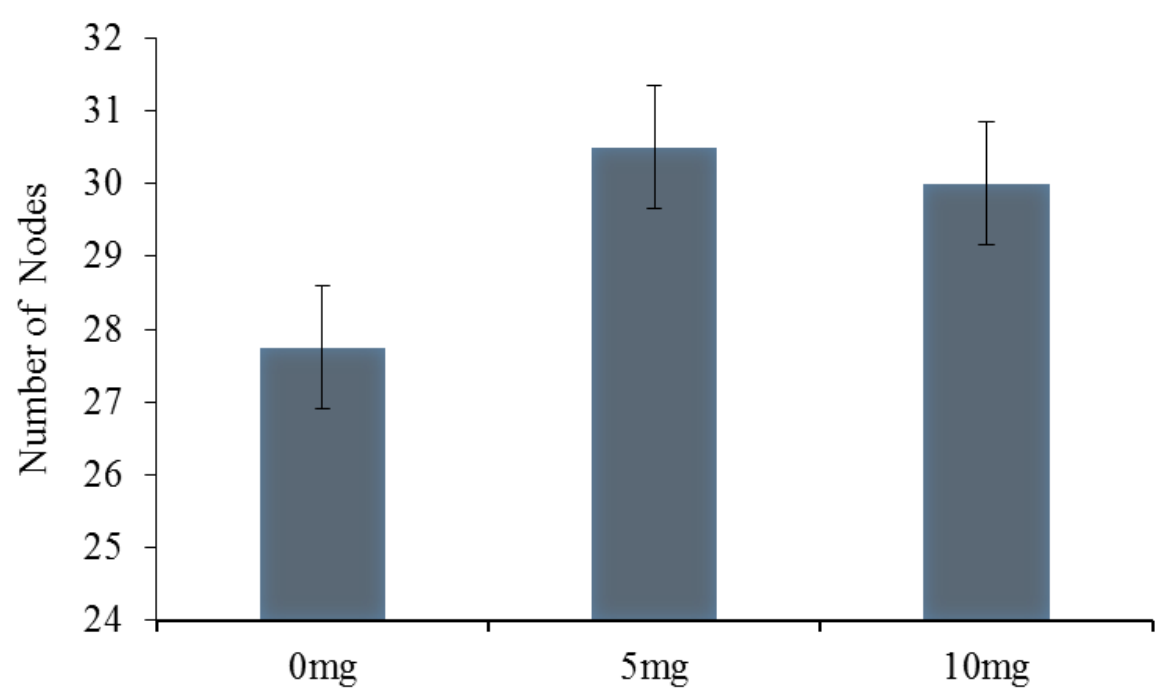

Figure 34. Quantification of the nodes on the basal and apical dendrites of CA3 pyramidal neurons after injections with different doses of NBQX and training on the MWM. No main effect of dose was found.

Number of branch ends of the apical and basal dendrites, plotted in Figure 35, increased with dose; $0 \mathrm{mg}(m=36.5, s=18.08), 5 \mathrm{mg}(m=39, \mathrm{~s}=4.83)$, and 10mg $(m=39.5, s=7.41)$. A one-way ANOVA revealed no significant difference between the groups $\left(F_{(2,9)}=.076, p=\right.$ 0.927). 


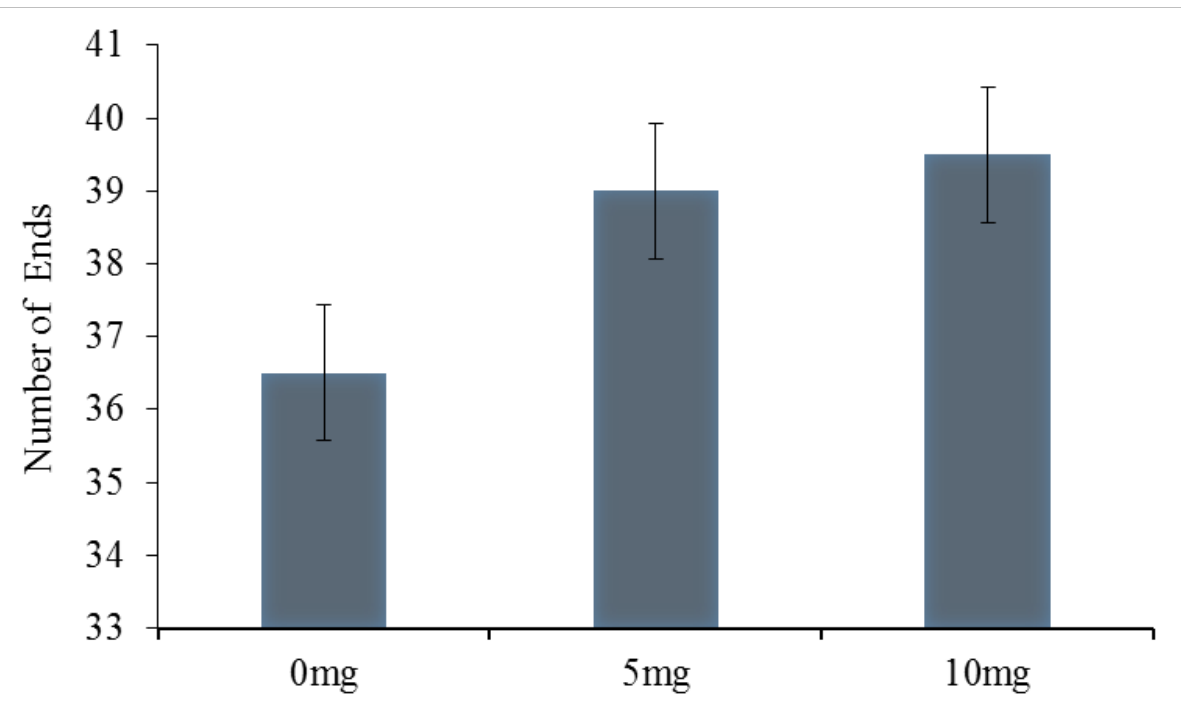

Figure 35. Quantification of the branch ends of the apical and basal dendrites of CA3 pyramidal neurons after injections with different doses of NBQX and training on the MWM. No main effect of dose was found.

\section{Length $(\mu \mathrm{m})$}

A Welch ANOVA, a robust test of equality of means, was conducted to determine if length of the CA3 pyramidal neuron was different between the groups (Figure 36). No main effect of dose was found, $\left(F_{(2,4.89)}=1.79, p=0.260\right)$. Means of the length of the neurons showed a decrease with dose: $0 \mathrm{mg}(m=10412.7, s=5521.36), 5 \mathrm{mg}(m=6056.3, s=4042.16)$ and $10 \mathrm{mg}$ $(m=4682.65, s=1729.09)$. 


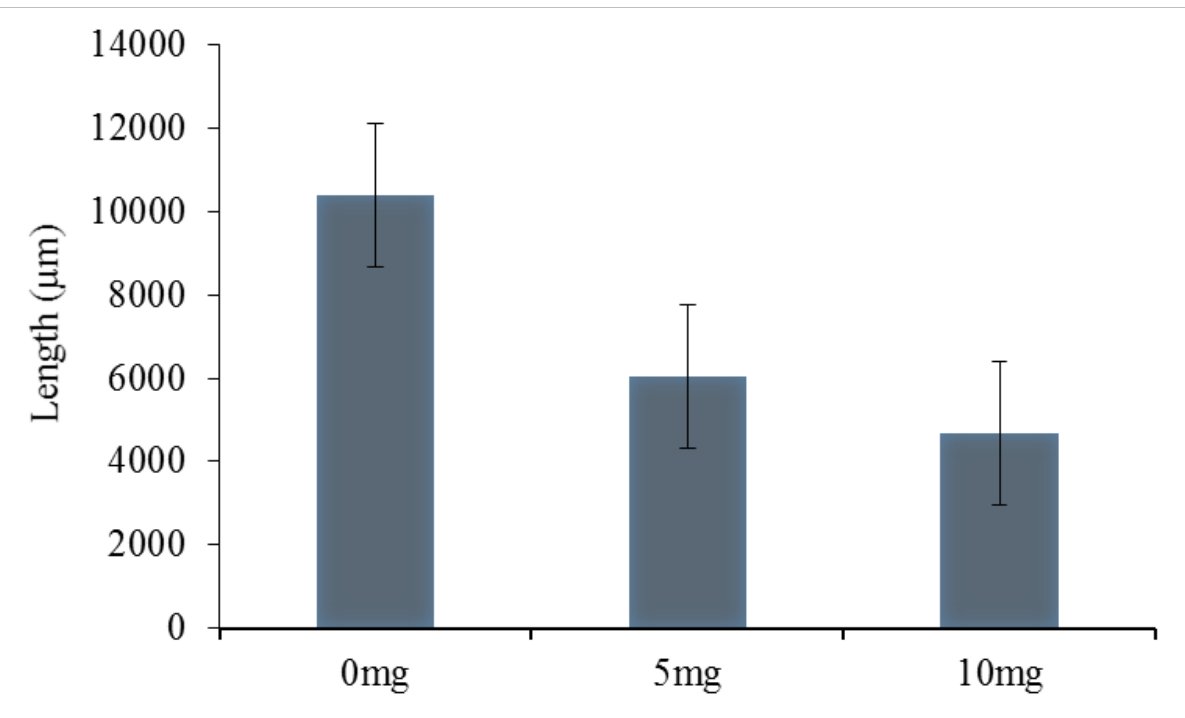

Figure 36. Length of the CA3 pyramidal neurons measured in $(\mu \mathrm{m})$ after injection with different doses of NBQX and training on the MWM. No main effect of dose was found.

For a further investigation, Welch ANOVA was conducted to determine if there was a difference between groups in the length of apical dendrites, plotted in Figure 37, and basal dendrites, plotted in Figure 36. Length of apical dendrites appeared to differ between the groups. Length was greater in the $5 \mathrm{mg}$ group $(m=3742.72, s=3521.11)$ compare to $0 \mathrm{mg}$ group ( $m=$ 2978.22, $s=2500.92)$. It was lower in the 10mg group $(m=1750.5, s=687.74)$ when compared with 0 and $5 \mathrm{mg}$ groups. However, the differences between groups were not statistically significant, $\left(F_{(2,4.1)}=0.881, p=0.476\right)$. 


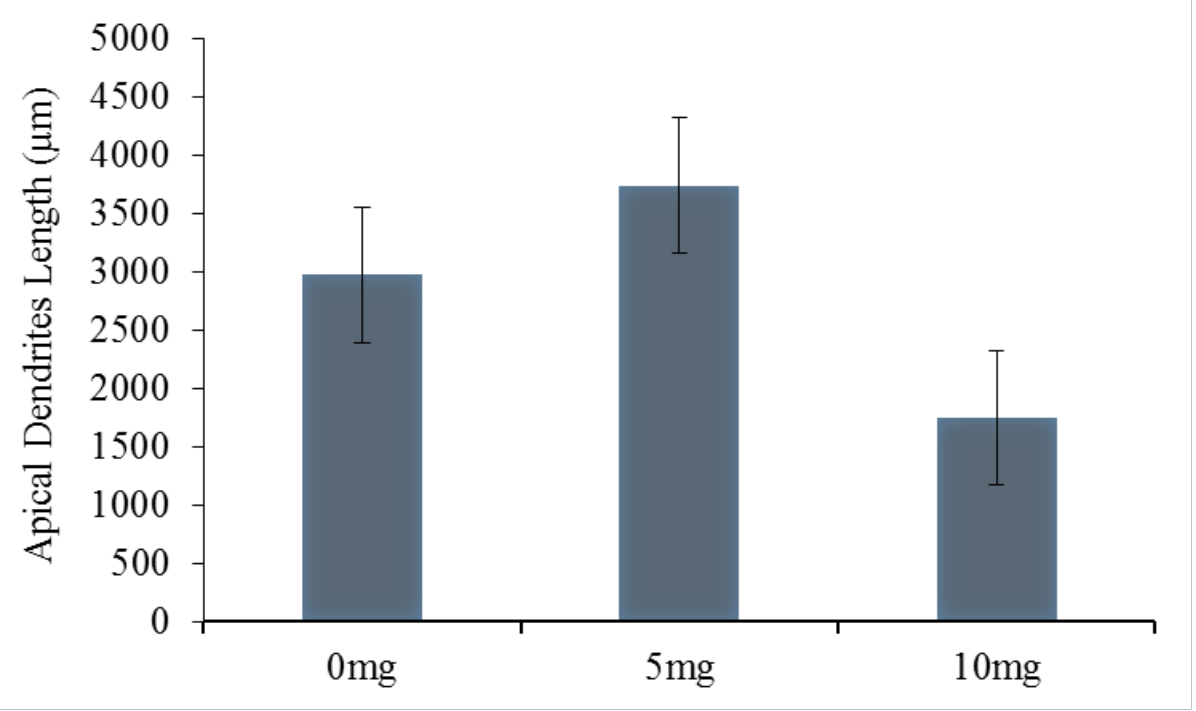

Figure 37. Length of apical dendrites of the CA3 pyramidal neurons measured in $(\mu \mathrm{m})$ after injections with different doses of NBQX and training on the MWM. No main effect of NBQX dose was found.

Basal dendrites were longest in the 0mg group $(m=7415.65, s=5360.03)$, and shortest in the 5mg group ( $m=2292.82, s=756.80)$ (Figure 38). Differences between groups were not statistically significant, $\left(F_{(2,4.85)}=1.75, p=0.267\right)$. 


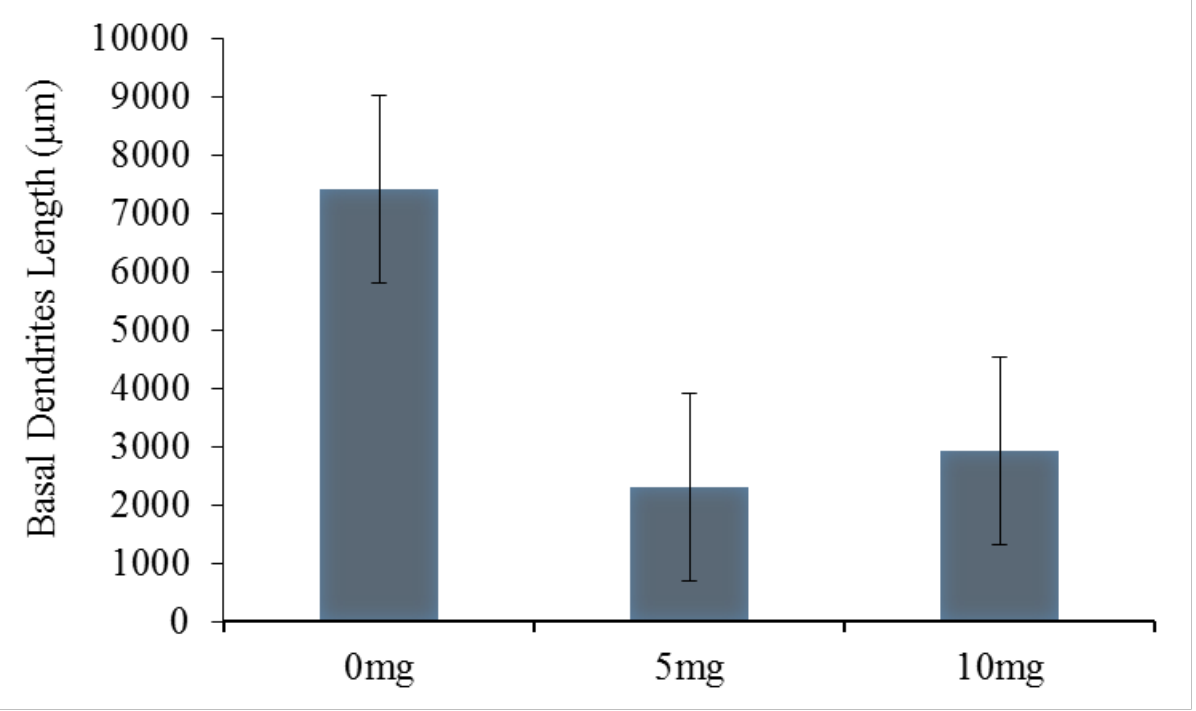

Figure 38. Length of the basal dendrites of CA3 pyramidal neurons measured in $(\mu \mathrm{m})$ after injection with different doses of NBQX and training on the MWM. No main effect of dose was found.

Surface, volume and complexity:

Surface of the CA3 pyramidal neurons, plotted in Figure 39, was greater in the 0mg group $(m=2374.08, s=1261.97)$ when compared to the $5 \mathrm{mg}$ group $(m=1376.06, s=907.41)$ and the 10mg group $(m=1074.03, s=389.04)$. However, differences were not statistically significant, $\left(F_{(2,4.88)}=1.77, p=0.264\right)$. 


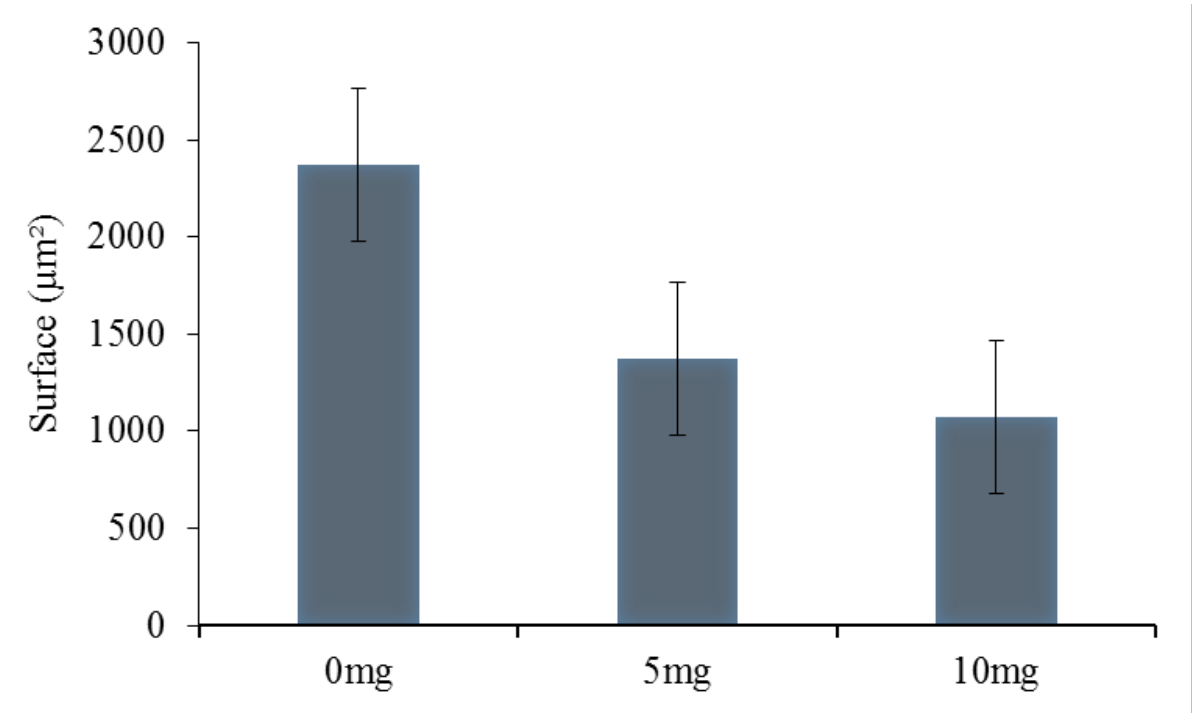

Figure 39. Surface of the CA3 pyramidal neurons measured in $\left(\mu \mathrm{m}^{2}\right)$ after injection with different doses of NBQX and training on the MWM. No main effect of dose was found.

Similarly, volume of the CA3 pyramidal neurons, plotted in Figure 40, was greater in the 0mg group $(m=43.15, s=23.03)$ compared to the $5 \mathrm{mg}(m=24.96, s=16.32)$ and the $10 \mathrm{mg}$ groups $(m=19.60, s=6.96)$. However, differences were not statistically significant, $\left(F_{(2,4.87)}=\right.$ $1.75, p=0.267)$. 


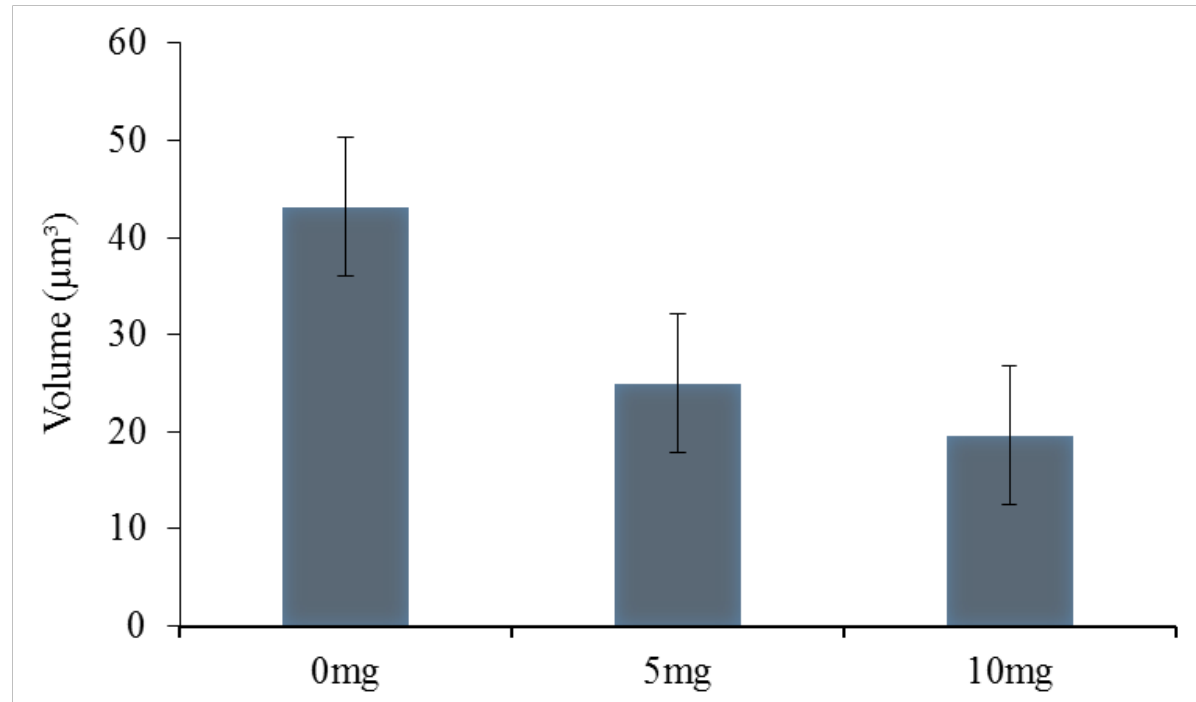

Figure 40. Volume of the CA3 pyramidal neurons measured in $\left(\mu \mathrm{m}^{3}\right)$ after injection with different doses of NBQX and training on the MWM. No main effect of dose was found.

Complexity, as plotted in Figure 41, reduced dramatically in the 5mg group ( $m=$ 177417.84, $s=96888.63)$ compared to the $0 \mathrm{mg}$ group $(m=699247.98, s=1063455.95)$. It was further decreased in the 10mg group ( $m=112285.53, s=22157.19)$. However, Welch ANOVA revealed no statistically significant difference between the groups, $\left(F_{(2,4.2)}=1.26, p=0.372\right)$. 


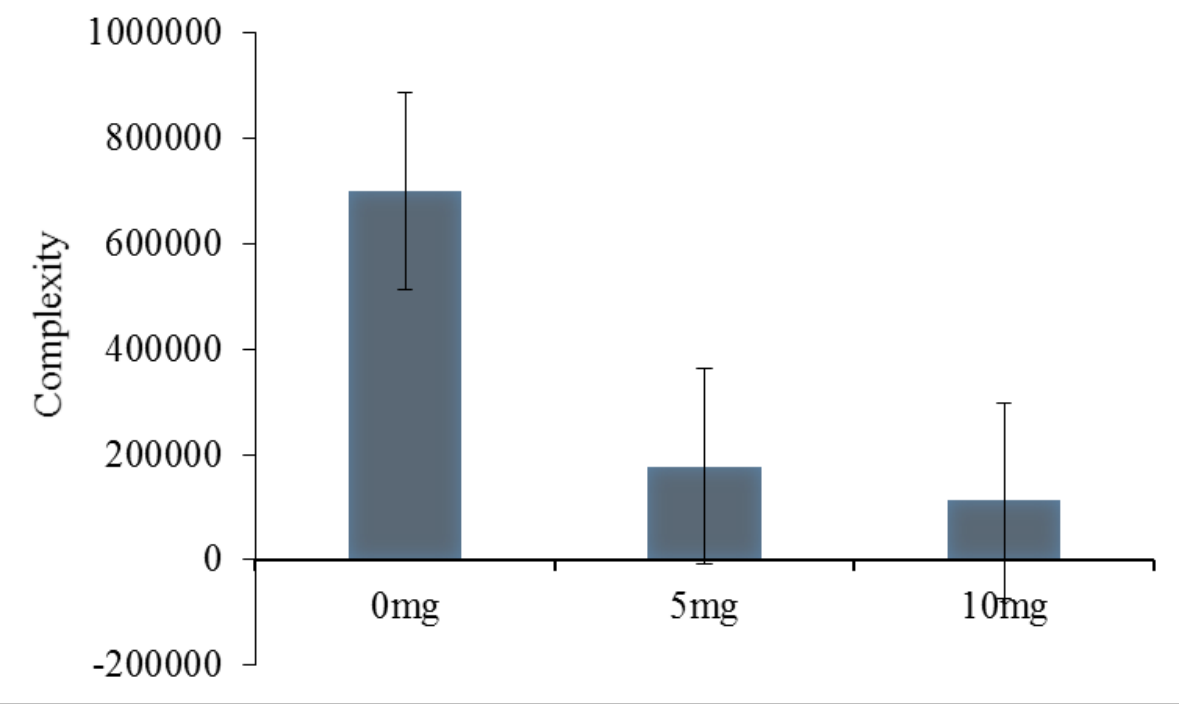

Figure 41. Complexity of CA3 pyramidal neurons measured in $\left(\mu \mathrm{m}^{3}\right)$ after injections with different doses of NBQX and training on the MWM. No main effect of dose was found.

\section{Spine quantification}

$\underline{\text { Entire neuron }}$

One-way ANOVA was conducted to determine spine number and density in the entire CA3 pyramidal neuron. Total number of spines, plotted in Figure 42, increased with dose. It was lowest in the 0mg group $(m=412.5, s=288.17)$, and increased in the $5 \mathrm{mg}(m=1020.5, s=$ 332.74) and 10mg groups ( $m=1082.5, s=544.81)$. However, no significant difference between groups was found $\left(F_{(2,9)}=3.35, p=.082\right)$. 


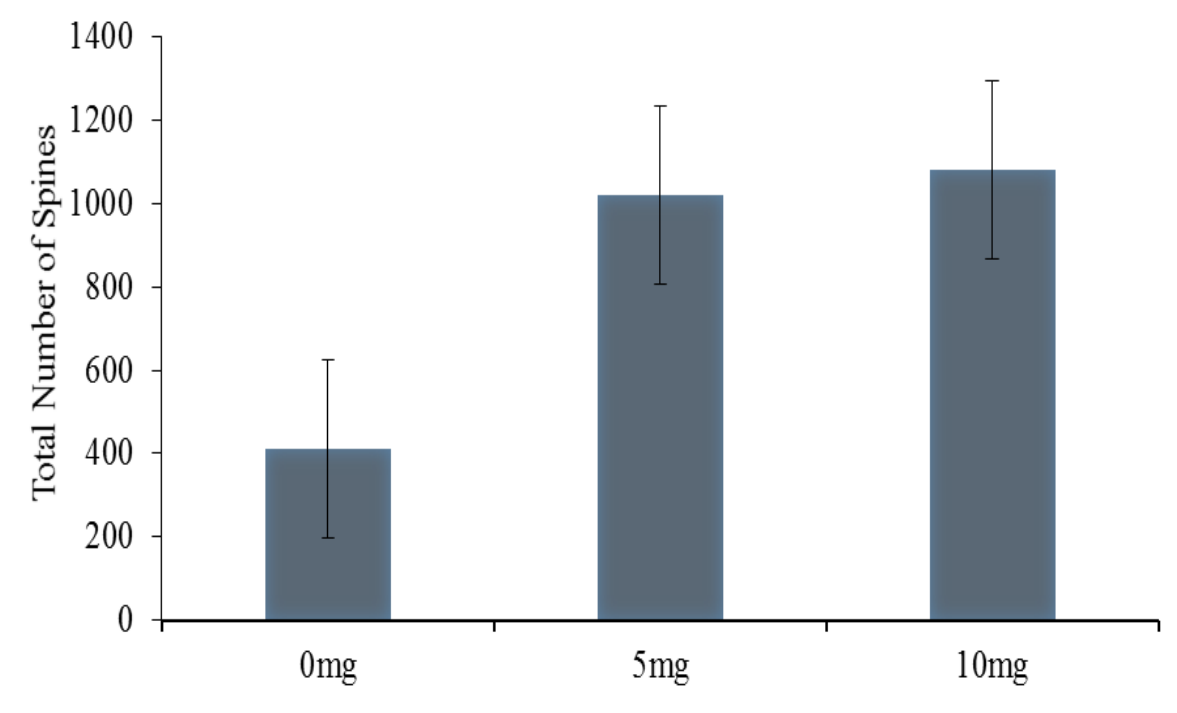

Figure 42. Quantification of spines on the entire CA3 pyramidal neurons after injections with different doses of NBQX and training on the MWM. No main effect of dose was found.

Total spine density measured as the total number of spines divided by the total length of the neuron, was obtained and plotted in Figure 43. No main effect of dose was found $\left(F_{(2,9)}=\right.$ $2.70, p=0.120)$. Mean density increased with dose from $0 \mathrm{mg}(m=0.03, s=0.01), 5 \mathrm{mg}(m=$ $0.23, s=0.15)$ to $10 \mathrm{mg}(m=0.29, s=0.23)$. 


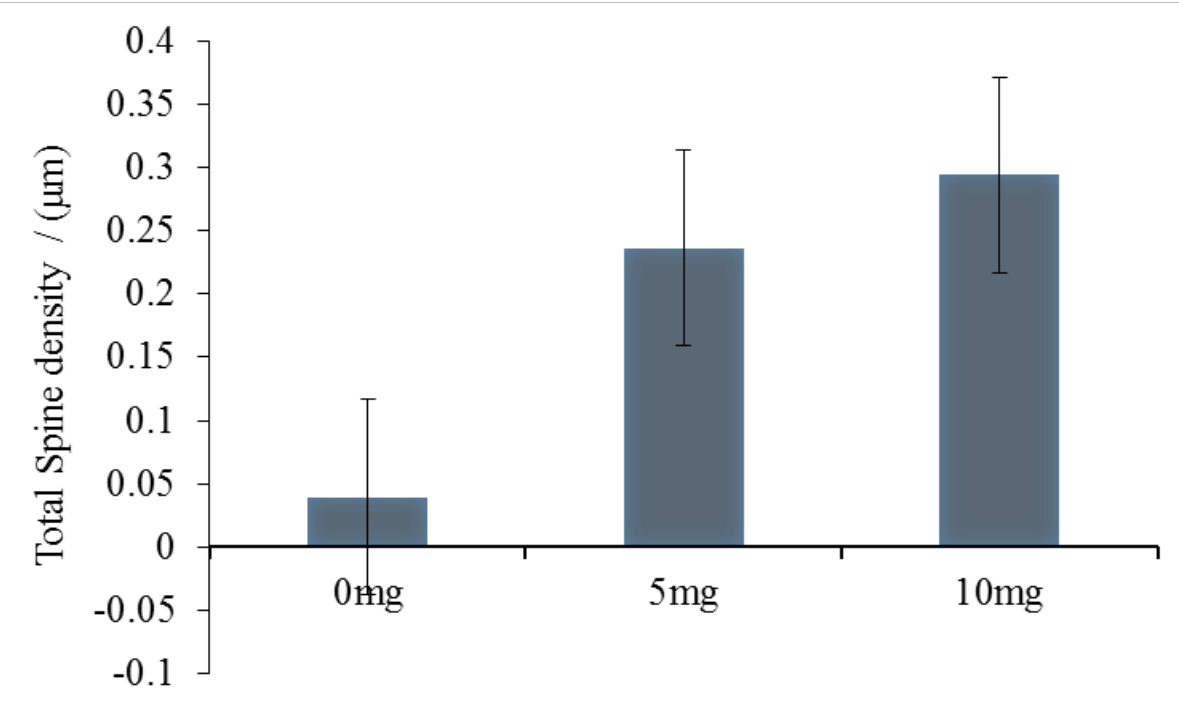

Figure 43. Spine density per $\mu \mathrm{m}$ of CA3 pyramidal neurons after injections with different doses of NBQX and training on the MWM. No main effect of dose was found.

Total spine density was obtained by taking the total number of spines divided by the number of dendrites (both apical and basal; Figure 44). The total density increased with dose from $0 \mathrm{mg}(m=79.15, s=48.09)$ to $5 \mathrm{mg}(m=149.39, s=56.68)$, and was almost the same in the 10mg group $(m=150.98, s=78.35)$. One-way ANOVA revealed no significant difference between the groups, $\left(F_{(2,9)}=1.73, p=0.231\right)$. 


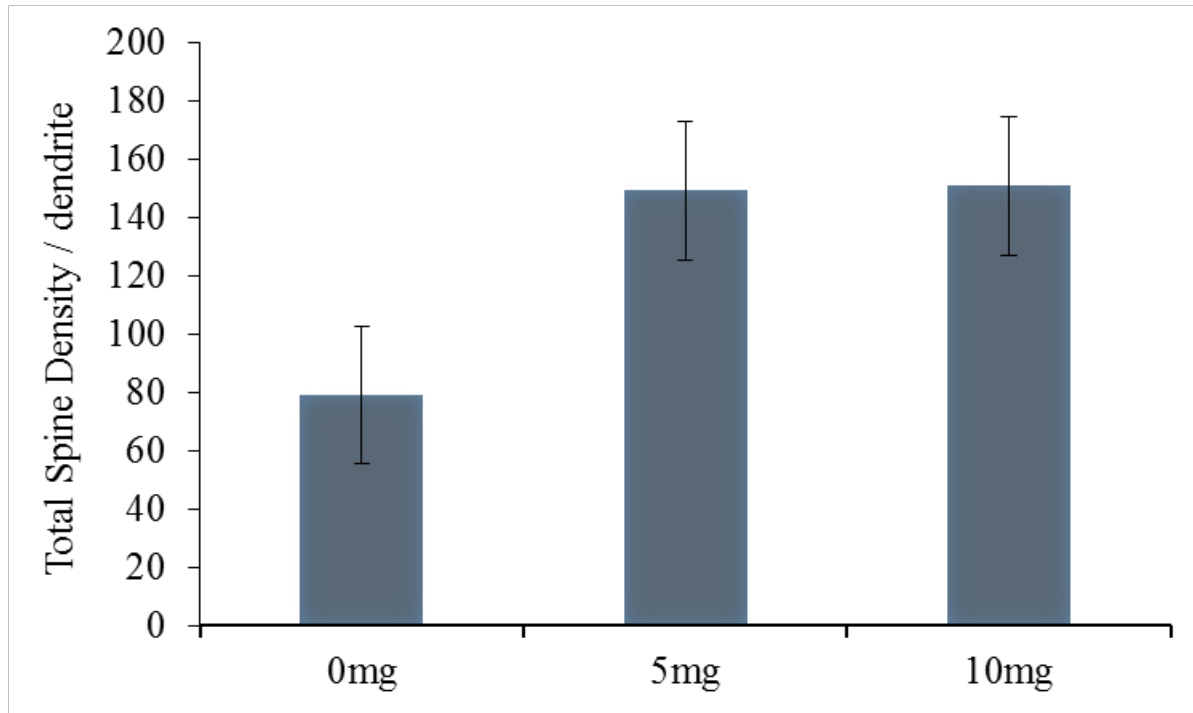

Figure 44. Spine density per dendrite (apical and basal) of the CA3 pyramidal neurons after injections with different doses of NBQX and training on the MWM. No main effect of dose was found.

$\underline{\text { Apical spines }}$

A Welch ANOVA was conducted to determine if there was a difference between groups in spine number and density on the apical dendrites. Means of the apical spines, plotted in Figure 45 , increased with does: $0 \mathrm{mg}(m=138.25, s=134.02), 5 \mathrm{mg}(m=269.5, s=125.08)$, to $10 \mathrm{mg}(m$ $=388, s=326.15)$. However, no significant difference was found between groups $\left(F_{(2,5.56)}=\right.$ $1.41, p=0.318)$. 


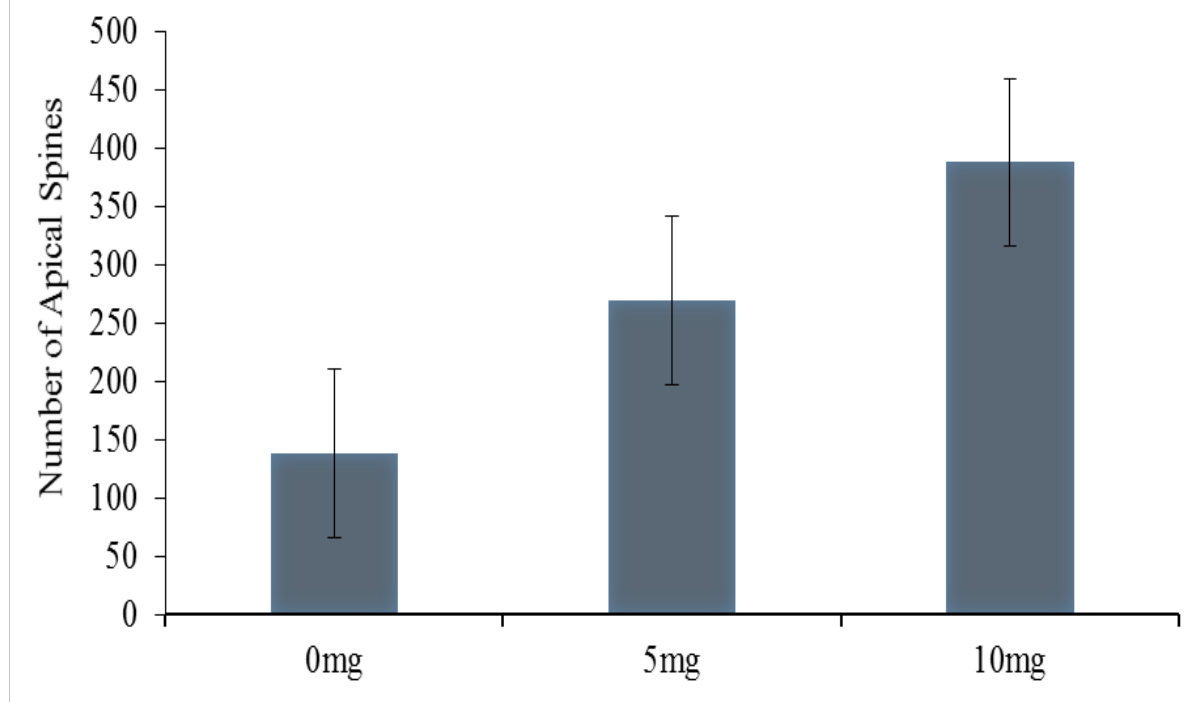

Figure 45. Quantification of spines on apical dendrites of CA3 pyramidal neurons after injections with different doses of NBQX and training on the MWM. No main effect dose was found.

Apical spine density was obtained by taking the number of spines on the apical dendrites divided by length of apical dendrites (Figure 46). Means showed an increase with dose: $0 \mathrm{mg}(\mathrm{m}$ $=0.11, s=0.18), 5 \mathrm{mg}(m=0.15, s=0.11)$ to $10 \mathrm{mg}(m=0.24, s=0.21)$. However, one-way ANOVA revealed no significant difference between groups $\left(F_{(2,9)}=0.535, p=0.603\right)$. 


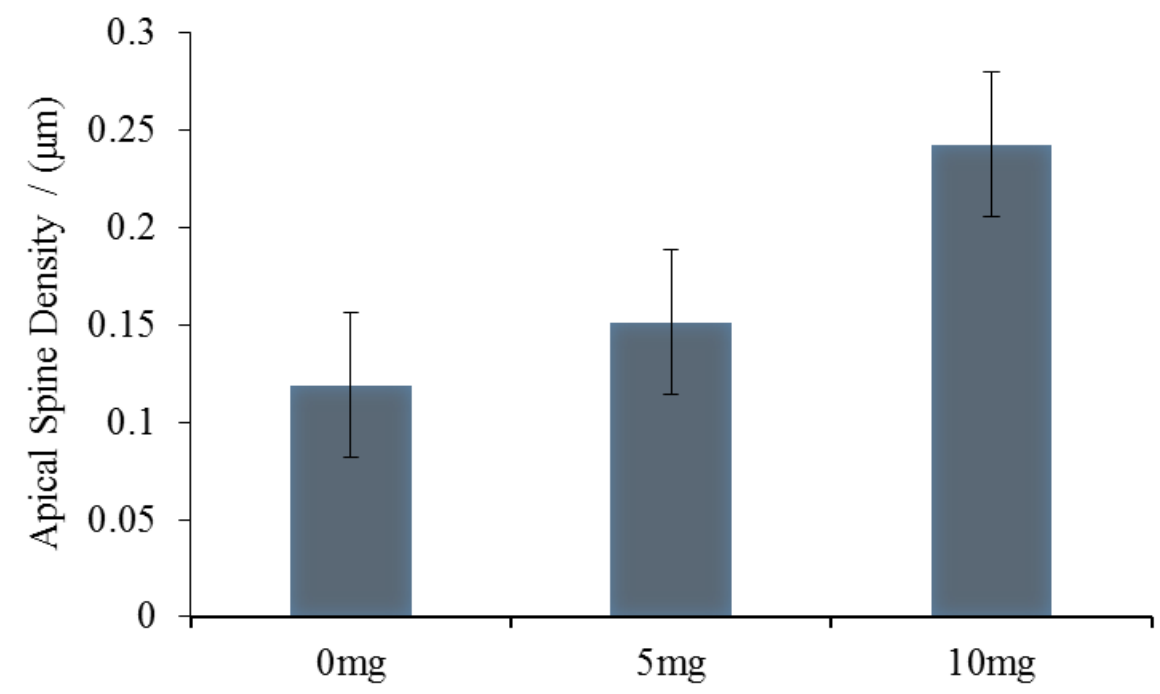

Figure 46. Spine density per $\mu \mathrm{m}$ of apical dendrites of CA3 pyramidal neurons after injections with different doses of NBQX and training on the MWM. No main effect of dose was found.

Apical spine density was obtained by taking the number of spines on the apical dendrites divided by number of apical dendrites (Figure 47). Means increased from 0mg $(m=138.25, s=$ $134.02)$ to $5 \mathrm{mg}(m=226.5, s=120.41)$, and decreased slightly in the 10mg group $(m=223, s=$ 132.23). One-way ANOVA revealed no significant difference between groups $\left(F_{(2,9)}=0.66, p=\right.$ $0.569)$. 


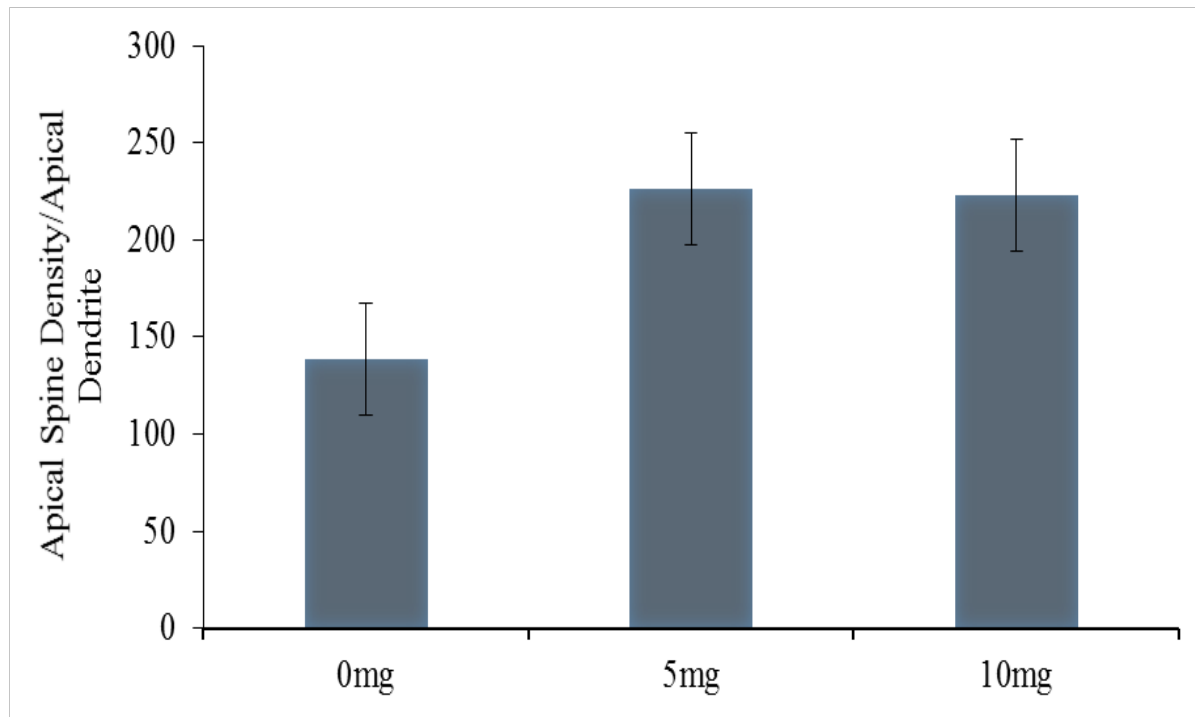

Figure 47. Spine density per apical dendrite of CA3 pyramidal neurons after injections with different doses of NBQX and training on the MWM. No main effect of dose was found.

\section{$\underline{\text { Basal spines }}$}

One-way ANOVA was conducted to determine if there was a difference between groups in spine number and density on the basal dendrites. Basal spine number, plotted in Figure 48, increased from $0 \mathrm{mg}(m=274.25, s=273.46)$ to $5 \mathrm{mg}(m=711.5, s=395.85)$, then decreased slightly in the 10mg group $(m=694.5, s=267.78)$. However, one-way ANOVA revealed no significant difference between groups $\left(F_{(2,9)}=2.42, p=0.143\right)$. 


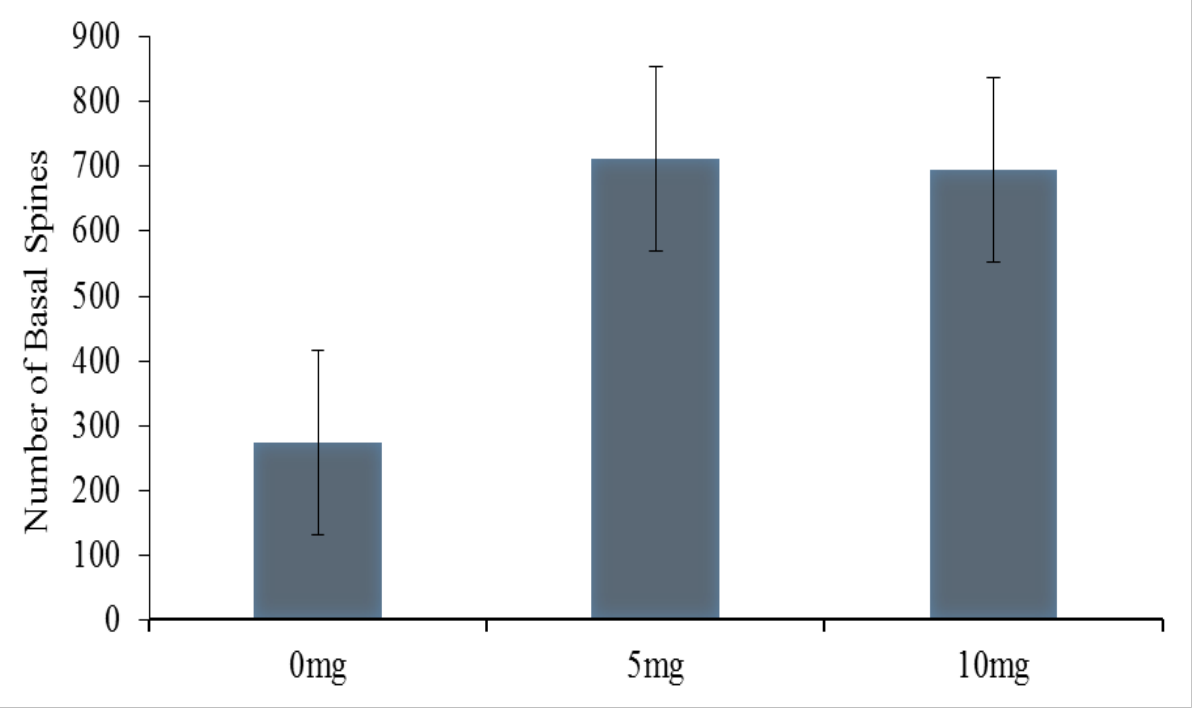

Figure 48. Quantification of spines on basal dendrites of CA3 pyramidal neurons after injections with different doses of NBQX and training on the MWM. No main effect dose was found.

Basal spine density was obtained by taking the number of spines on the basal dendrites divided by the length of basal dendrites (Figure 49). Means increased sharply with dose from $0 \mathrm{mg}(m=0.04 . s=0.02)$ to $5 \mathrm{mg}(m=0.3, s=0.12)$, then increased slightly in the $10 \mathrm{mg}$ group ( $m=0.33, s=0.27$ ). However, no significant difference was found between groups as revealed by one-way ANOVA $\left(F_{(2,9)}=3.32, p=0.083\right)$. 


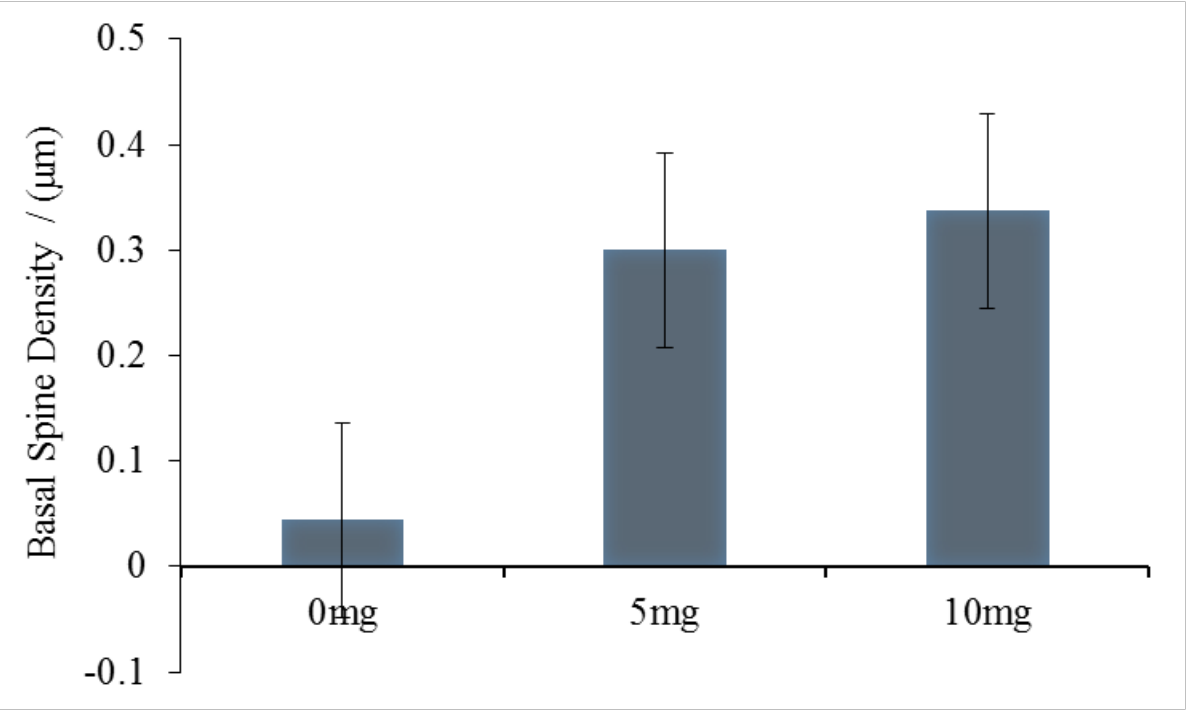

Figure 49. Spine density per $\mu \mathrm{m}$ of basal dendrites of CA3 pyramidal neurons after injections with different doses of NBQX and training on the MWM. No main effect of dose was found.

Basal spine density was obtained by taking the number of spines on the basal dendrites divided by number of basal dendrites (Figure 50). Basal spine density almost doubled from $0 \mathrm{mg}$ $(m=60.23, s=52.42)$ to $5 \mathrm{mg}(m=128.82, s=86.72)$, then decreased slightly in the $10 \mathrm{mg}$ group $(m=127.24, s=61.23)$. One-way ANOVA revealed no significant difference between groups $\left(F_{(2,9)}=1.31, p=0.316\right)$. 


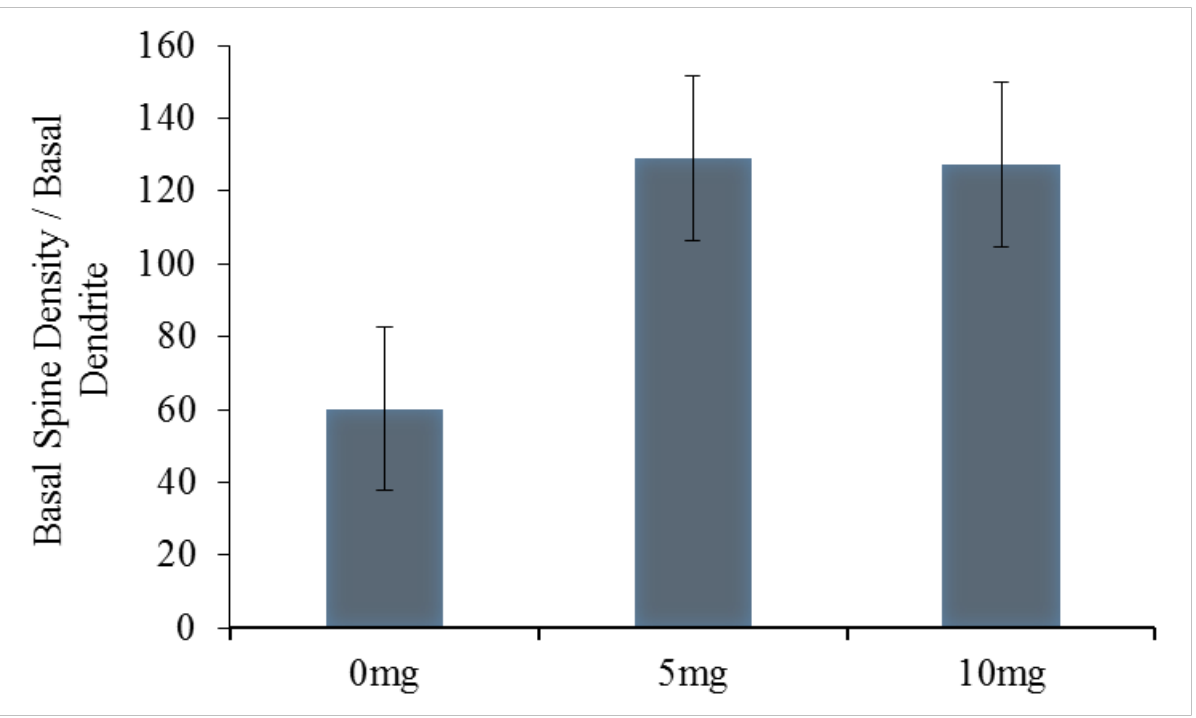

Figure 50. Spine density per basal dendrite of $\mathrm{CA} 3$ pyramidal neurons after injections with different doses of NBQX and training on the MWM. No main effect of dose was found.

\section{Thorny excrescence analysis}

Thorny excrescences (TE) on CA3 neurons from the groups were quantified from three areas; cell body, apical dendrites and basal dendrites (see Figure 51). Twenty four neurons were included in the analysis (8 neurons per group). One-way ANOVA and Welch ANOVA were used to compare thorny excrescence means between the three groups. 

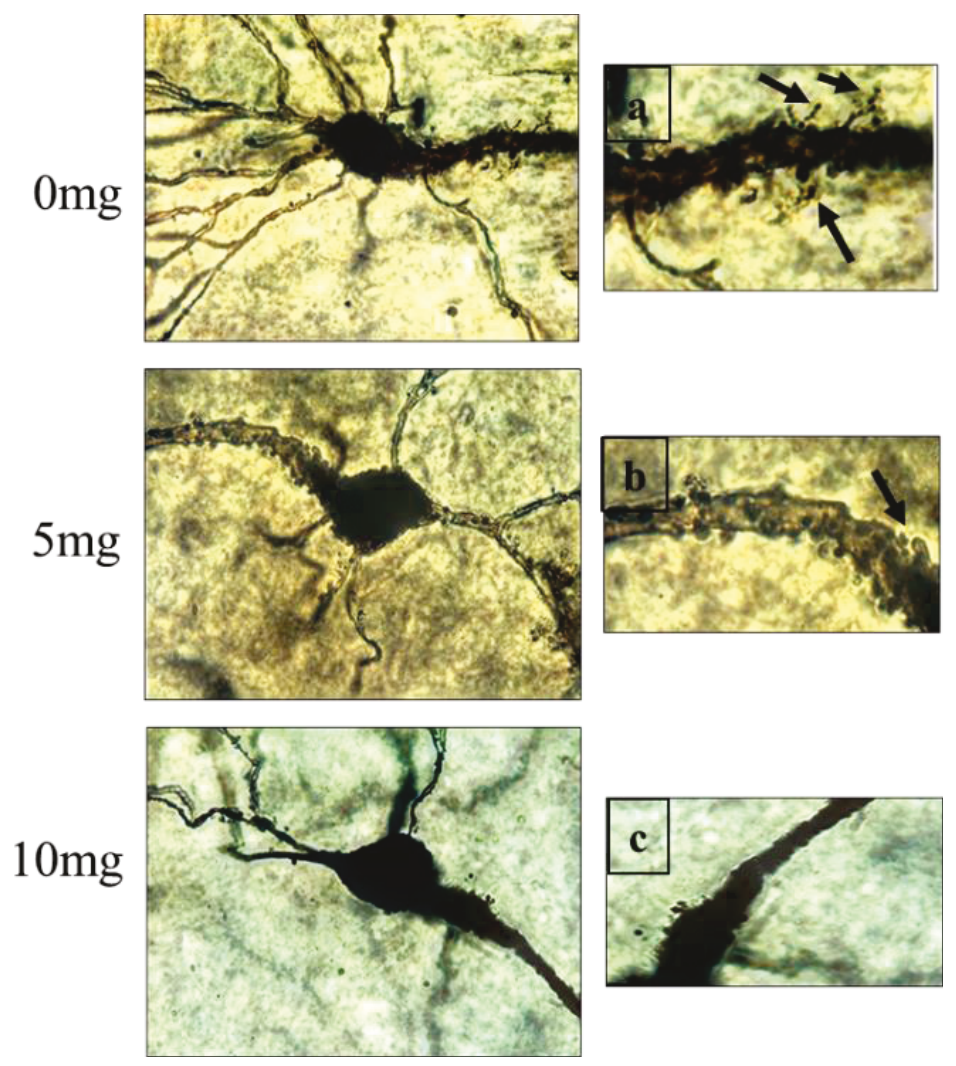

Figure 51. Representative images for Golgi impregnation of TE on CA3 pyramidal neurons in groups: $0 \mathrm{mg}, 5 \mathrm{mg}$ and $10 \mathrm{mg}$. Images: a, b and $\mathbf{c}$ represent $\mathrm{TE}$ on the apical dendrites of CA3 neurons, proximal to the cell bodies, in $0 \mathrm{mg}, 5 \mathrm{mg}$ and $10 \mathrm{mg}$ respectively. Images were taken at $100 \mathrm{X}$.

A Welch ANOVA on TE on the entire neuron (cell body, apical and basal dendrites) revealed a statistically significant main effect of dose $\left(\mathrm{F}_{(2,10.42)}=4.39, p<0.05\right)$. Means of the total TE, plotted in Figure 52, showed a greater number of TE in the 0mg group $(m=11.87, s=$ 11.60) compared to the $5 \mathrm{mg}$ group $(m=10.37, s=7.76)$ and the $10 \mathrm{mg}$ group $(m=3.37, s=$ 2.55). However, Games-Howell post-hoc tests revealed no significant differences between the groups. 


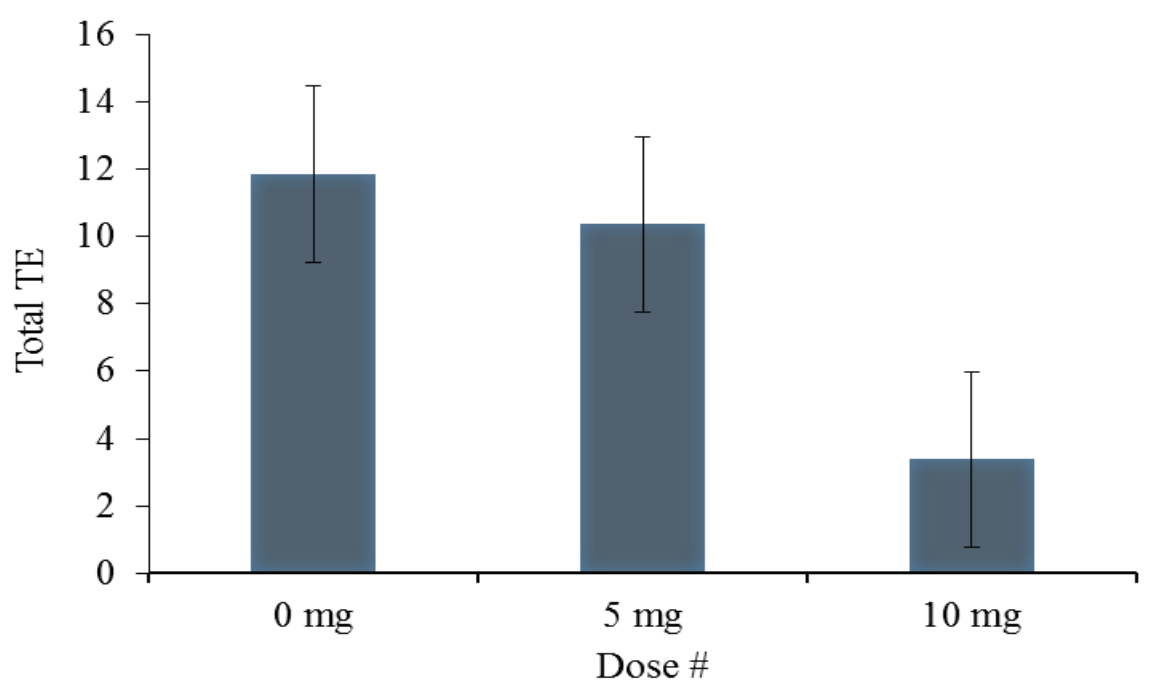

Figure 52. Quantification of TE on the CA3 pyramidal neurons after injection with different doses of NBQX and training on the MWM. (\#) indicates a main effect of dose $(p<0.05)$.

Welch ANOVA on the number of TE on the apical dendrites revealed no main effect of dose, $\left(F_{(2,12.69)}=2.52, p=0.119\right)$. Means of apical TE, plotted in Figure 53, showed a decrease in the $5 \mathrm{mg}$ group $(m=3.25, s=2.31)$ and a further decrease in the 10mg group $(m=1.62, s=$ 1.92) compared to the $0 \mathrm{mg}$ group $(m=6.37, s=6.52)$. 


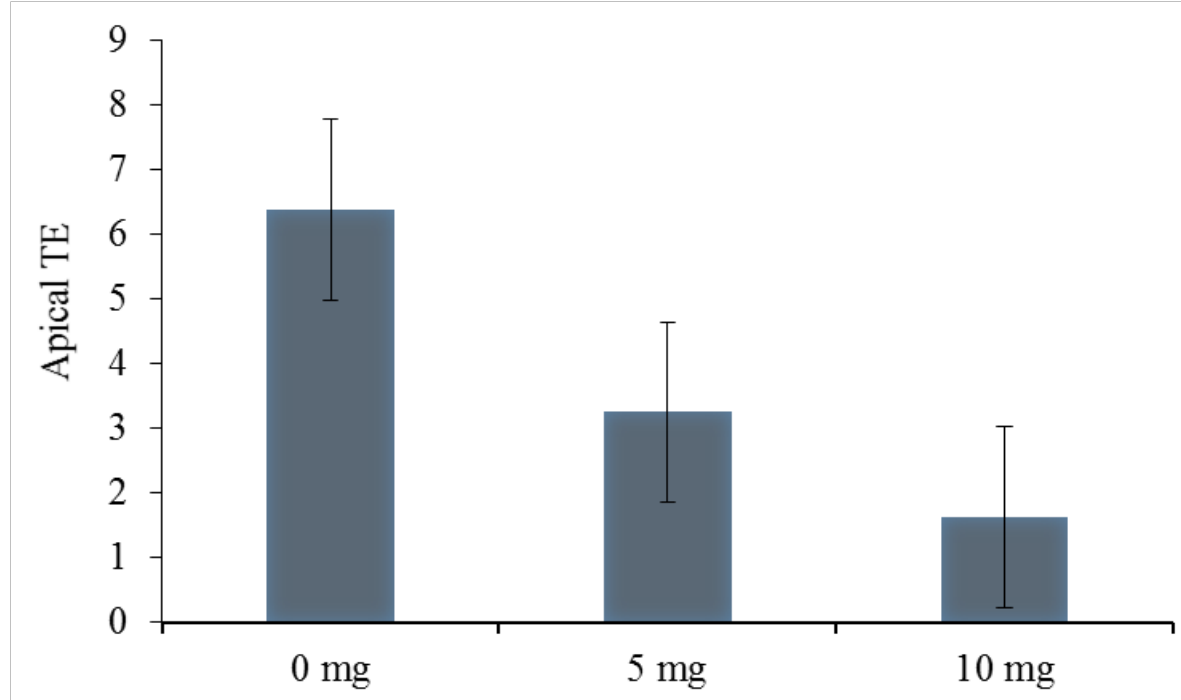

Figure 53. Quantification of TE on the apical dendrites of $\mathrm{CA} 3$ pyramidal neurons after injections with different doses of NBQX and training on the MWM. No main effect of dose was found.

A Welch ANOVA on the number of TE on the basal dendrites revealed a main effect of dose, $\left(F_{(2,11.07)}=6.05, p<0.05\right)$. Games-Howell post-hoc tests revealed significant differences between the 5mg $(m=3.87, s=2.99)$ and $10 \mathrm{mg}(m=0.5, s=1.06)(p<0.05)$ groups (Figure $54)$. 


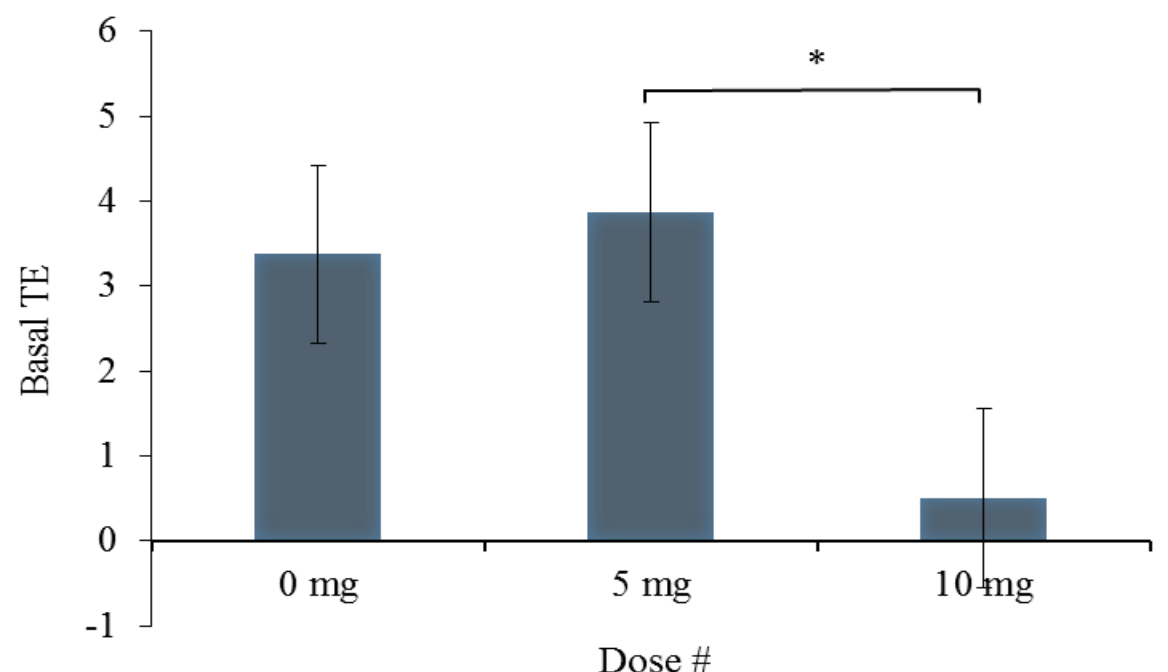

Figure 54. Quantification of TE on the basal dendrites of CA3 pyramidal neurons after injections with different doses of NBQX and training on the MWM. A main effect of dose was found $\left.(p<0.05) .{ }^{*}\right)$ indicates a significant differences between $5 \mathrm{mg} / \mathrm{mg}$ and $10 \mathrm{mg}(p<0.05)$.

One-way ANOVA on the number of TE on the cell body revealed no main effect of dose $\left(F_{(2,21)}=1.04, p=0.370\right)$. Means of the cell body TE, plotted in Figure 55, showed the highest number of TE in the $5 \mathrm{mg}$ group $(m=3.25, s=3.73)$ followed by $0 \mathrm{mg}$ group $(m=2.12, s=2.69)$, and lowest in the 10mg group $(m=1.25, s=1.38)$. 


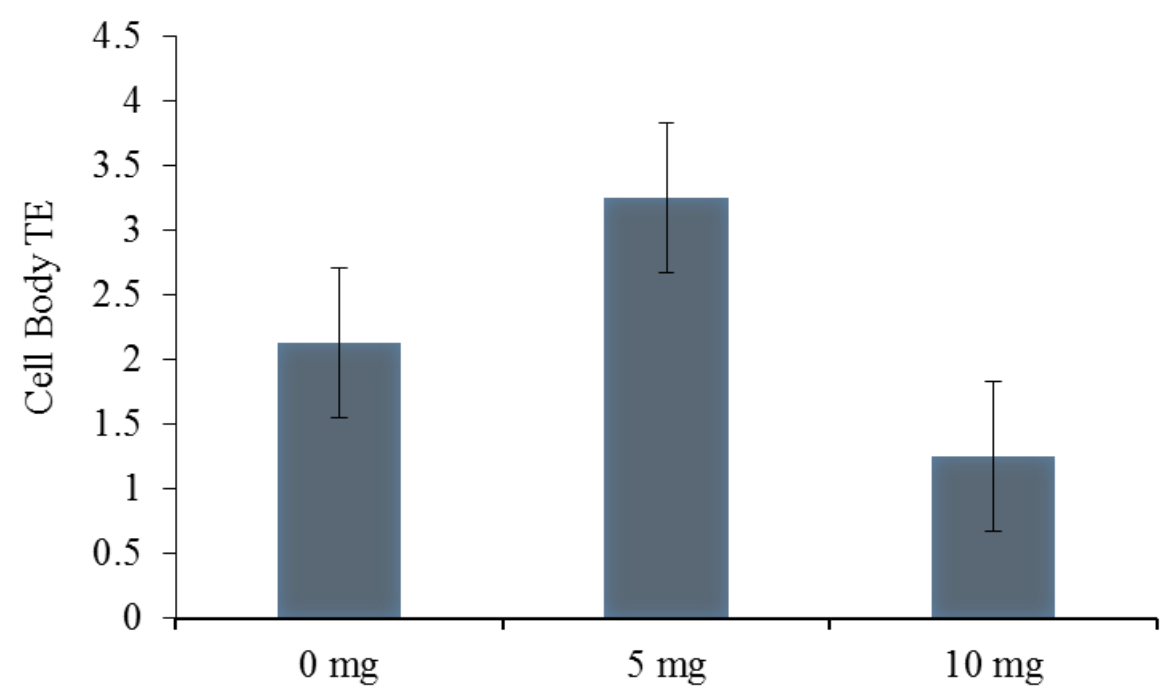

Figure 55. Quantification of TE on cell bodies of the CA3 pyramidal neurons after injections with different doses of NBQX and training on the MWM. No main effect of dose was found. 


\section{Discussion}

\section{Summary of the results}

In Experiment 1, the assessment of morphological changes in CA3 neurons between p18 and p24 revealed different rates and patterns of development of individual components of these neurons. In general, the measurements taken were lowest on $\mathrm{p} 18$, and they peaked at different ages. For example, total and basal dendritic lengths, surface area and volume increased gradually with age peaking at $\mathrm{p} 24$. However, number of apical dendrites peaked at p22 and decreased slightly at p24 suggesting pruning of CA3 apical dendrites. Complexity peaked at p20, decreased at 22 then increased slightly at p24. Number of nodes, ends and basal dendrites peaked at p20 then decreased with age to p24 suggesting retraction of these components. For the number of basal dendrites, there were fewer noted at p24 than p18. Hence, number of basal dendrites was one measure that showed reductions during this developmental period. Finally, cell body areas showed minimum changes between $\mathrm{p} 18$ and p20, increased at p22 and remained similar at p24.

Spine quantification from different areas of CA3 neurons also showed different rates and patterns of change between $\mathrm{p} 18$ and $\mathrm{p} 24$. In all spine measurements, $\mathrm{p} 18$ showed the lowest in number and density except for apical density per $\mu \mathrm{m}$. Apical spine number and apical density per $\mu \mathrm{m}$ peaked at p20 then decreased steadily with age. Total and basal spine numbers and densities (per $\mu \mathrm{m}$ and per dendrite) increased with age, peaked at $\mathrm{p} 22$ then declined slightly at $\mathrm{p} 24$. Total and basal densities per dendrite were lower at p24 than p18. An exception was the apical density per $\mu \mathrm{m}$, being highest at $\mathrm{p} 18$ then decreasing with age. The decrease in spines at $\mathrm{p} 24$ suggests pruning of synapses at this age. This developmental pattern is similar to that described by 
Holahan et al. (2007) who reported a retraction in the number of mossy fiber axonal inputs to CA3 neurons at p24 in Wistar rats (Holahan, Honegger, \& Routtenberg, 2007).

Thorny excrescences showed a significant increase with age in all parts of the neurons (total, apical, basal and the cell body). As discussed above, spine density and number showed a decrease at p24 while thorny excrescences became larger and increased in number. Converging lines of evidence from behavioral studies point to the postnatal period from p16 to p24 as a sensitive developmental timeframe for spatial behavioral function to emerge. Improved performance on a number of spatial tasks during this timeframe has been noted (Akers, Candelaria-Cook, Rice, Johnson, \& Hamilton, 2009, 2011; Akers, Candelaria, \& Hamilton, 2007; Akers \& Hamilton, 2007; Blair et al., 2013; Brown \& Whishaw, 2000; Douglas, Peterson, \& Douglas, 1973; Dumas, 2004; Green \& Stanton, 1989; Holahan, Honegger, \& Routtenberg, 2007; Jablonski, Schiffino, \& Stanton, 2012; Keeley, Wartman, Haeusler, \& Holahan, 2010; Langston et al., 2010; Rudy, 1993; Rudy \& Morledge, 1994; Rudy, Stadler-Morris, \& Albert, 1987; Smith, Macdonald, \& Holahan, 2011; Tonkiss, Shultz, \& Galler, 1992; Wartman et al., 2012; Wills, Cacucci, Burgess, \& O'Keefe, 2010) indicating a sensitive developmental period for spatial behavior to emerge between $\mathrm{p} 16$ and $\mathrm{p} 24$. The present anatomical results suggest that maturation of thorny excrescences on CA3 neurons may contribute to the developmental emergence of spatial behavioral function.

In Experiment 2, on the second day of training, there was a significant reduction in latency and thigmotaxis in the $0 \mathrm{mg}$ group compared to the $5 \mathrm{mg}$ and $10 \mathrm{mg}$ NBQX groups indicating that learning may have been impaired after NBQX treatment. On the third day of training, a significant reduction in latency and thigmotaxis in the $0 \mathrm{mg}$ group continued compared 
to the $5 \mathrm{mg}$ and $10 \mathrm{mg}$ NBQX groups. These results suggest that pretraining NBQX injections impaired the acquisition of the water maze task.

On the probe test day, the $0 \mathrm{mg}$ and $5 \mathrm{mg}$ groups spent more time swimming in the quadrant that contained the platform compared to the time they spent swimming in the opposite quadrant. This suggests that they remembered the location of the hidden platform. However, there was no difference in the time spent swimming in the target versus the opposite quadrant in the 10mg group, suggesting they did not remember the location of the platform.

Structural analysis of CA3 neurons from Experiment 2 showed varying results as a consequence of NBQX injections. NBQX injections were associated with decreased neuron complexity, volume, surface, total length, apical, and basal lengths. This suggests that blockade of AMPA receptors during water maze training negatively impacted the learning-associated changes in these neural elements.

Contrary to the measurements noted above, the cell body area, apical dendrites, basal dendrites, nodes, and ends showed an increase in number associated with NBQX injections. In general, spine analysis indicated that NBQX inhibited the normal pruning that occurs during development resulting in behavioral impairments on the MWM. The present results parallel those reported by Holahan et al. (2007) who reported an increase in the synapses between mossy fibers and CA3 neurons after administration of an NMDA receptor antagonist.

Thorny excrescences were reduced associated with NBQX treatment in the CA3 neurons. TE was significantly lower in the 10mg group, suggesting a dose-dependent effect on TE number. The dose-dependent reduction in TE on the backdrop of the increase in spines suggests 
an important contribution of $\mathrm{TE}$ in the processing of spatial information in the hippocampus through CA3 neurons.

Bringing together behavioral and structural analyses, behavioral impairments in the $5 \mathrm{mg}$ and $10 \mathrm{mg}$ groups were correlated with an increase in dendrites, spines, ends, and nodes on CA3 neurons. On the other hand, these groups showed a decrease in the overall number of thorny excrescences. These findings suggest that blockade of AMPA receptors may have interfered with the normal pruning of neural synapses during development and at the same time, interfered with training-associated stability of thorny excrescences. In this respect, fewer smaller synapses with stronger connections mediated by thorny excrescences would be indicative of facilitated spatial information processing.

\section{Dendritic Arborization and retraction}

Maturity and growth of dendritic arbors are crucial for normal functions of the nervous system. Any divergence from preprogrammed arborization can lead to drastic outcomes as detected following injuries or after aging (Dickstein, et al., 2007). Strength and quantity of neural signals received by a neuron are determined by the structure and distribution of the dendritic arborization. In addition, patterns of dendritic arborisation can determine the output characteristics of a neuron (Narayanan, Narayan \& Chattarji, 2005) and its computational abilities (McAllister, 2000). Studies have shown that dendritic growth and remodeling in various parts of the brain are notably progressive and responsive during development (Andrews, Thrasivoulou, Nesbit \& Cowen, 1996), aging (Markham, McKian, Stroup \& Juraska, 2005; Luebke \& Rosene, 2003), and under certain treatments and conditions (McAllister, 2000; Narayanan, Narayan \& Chattarji, 2005). 
In the present study, structural analysis during development (Experiment 1) revealed extensive remodeling of dendritic branches between $\mathrm{p} 18$ and $\mathrm{p} 24$. When rats of a similar age were treated with NBQX, trained on the water maze and tested and euthanized 3 weeks later (Experiment 2) remodeling was also evident. In Experiment 1, changes in dendritic remodeling were suggested to mediate the developmental emergence of spatial information processing. In Experiment 2, dendritic remodeling following AMPA receptor antagonist impaired the ability of rats to perform on the MWM. Dendritic remodeling, as revealed in both experiments, may be indicative of changes in function and circuitry of the CA3 neural locus.

Normal development of the brain not only requires synaptic outgrowth and expansion but, due to the seemingly overabundance of synapses, normal development requires selective retraction and pruning of a significant number of synapses (Chechik, Meilijson \& Ruppin, 1998). The decrease in the quantity of synapses that happens in early development and adolescence is a major phenomenon in brain development. Starting prenatally, synaptic density constantly increases, through the postnatal developmental period, until it peaks at a certain level and remains stable for a relatively brief period. Then, a pruning period starts and synapses are steadily eliminated. This course of action continues until adulthood when synapses and branches remain at a certain level until old age (Chechik, Meilijson \& Ruppin, 1998).

Pruning occurs in widespread brain areas and is associated with experience-dependent activity (Holtmaat \& Svoboda, 2009). The time range of synaptic retraction differs between various cortical regions, roughly in a dorsal to frontal sequence (Rakic, Bourgeois \& GoldmanRakic, 1994). The reduction in spine density does not lead to a reduction in total brain volume (Rakic, Bourgeois \& Goldman-Rakic, 1994). 
In the present thesis, differences in the growth and retraction rates and patterns were found between basal and apical dendrites of CA3 pyramidal neurons during the developmental period p18-p24. While some areas of the dendritic tree showed growth, other areas were more stable or showed retraction. For example, number of apical dendrites showed a high rate of growth and retracted slightly at p24, whereas number of basal dendrites started retracting at p20 and the reduction at p24 was significant. Length of apical dendrites fluctuated with age with an overall increase and length of basal dendrites showed a gradual increase with age. Basal spines at p20 were fewer than apical spines, but at p22 there were fewer apical spines than basal spines.

It has been found that remodeling under certain conditions can occur in some parts of the dendritic tree but not others (Narayanan, Narayan \& Chattarji, 2005). In Experiment 2 of the current study, parts of the CA3 neurons responded individually to the injections of NBQX. Length of basal dendrites showed a sharp drop in the $5 \mathrm{mg}$ group, while length of apical dendrites showed an increase. Number of apical dendrites increased in association with NBQX dose, but number of basal dendrites increased and then retracted slightly with the higher dose. Number of TE decreased with dose in apical and basal dendrites, but the decline in TE basal dendrites was significant.

Some have suggested that stronger synaptic inputs maintain a more stable structure of dendritic trees whereas weaker signals allow for rapid dendritic tree remodeling and growth $(\mathrm{Wu}$, Zou, Rajan \& Cline, 1999). In this study, the presence of TE are suggested to make stronger connections between CA3 and DG granule cells and contribute to more stable patterns of network activity. For example, basal dendrites showed relatively higher plasticity and weaker connections when compared to apical dendrites: Basal dendrite number exhibited a rapid increase at p20 and a significant drop at p24. Basal spine density was also significantly lower at 
p24, while TE were significantly higher. Apical dendrites, on the other hand, showed more stable patterns associated with a significant increase in TE at p22 and p24 when compared to basal dendrites. Number of apical dendrites increased gradually with age and declined slightly at p24. Spine density declined at $\mathrm{p} 24$. Changes in apical dendrite number and spines density were not significant suggesting a more stable network.

In Experiment 2, basal dendrites showed relatively rapid plasticity and weaker connections when compared to apical dendrites. Basal dendrites length sharply dropped after NBQX injections, spine density increased and TE were significantly reduced. Apical dendrite length slightly increased then decreased with a higher dose of NBQX, spine density increased, and TE decreased but not significantly. Therefore, in the current study it is suggested that the reduction in TE after NBQX injections led to the disturbance/impairment in only allocentric spatial learning and memory. Allocentric spatial learning and memory is a specific function of DG-CA3 neurons but no other circuits in the hippocampus as found in studies (Florian \& Roullet, 2004; Kesner, Hunsaker \& Warthen, 2008; Hunsaker, Lee \& Kesner, 2008; Hunsaker, Thorup, Welch \& Kesner, 2006; Holahan \& Routtenberg, 2011; Czéh, et al., 2001).

\section{LTP and structural remodeling}

Long-term potentiation (LTP) is a prolonged enhancement of a synaptic communication between two neurons that is typically induced by high-frequency repeated stimulation from the presynaptic neuron and activation of the post-synaptic neuron. The high-frequency signals trigger plasticity on the membrane of the postsynaptic neurons. Following LTP, a smaller presynaptic signal results in a stronger postsynaptic response. LTP is believed to be the cellular 
and molecular basis of learning and memory. Blocking LTP has been shown to block learning and memory (Nabavi, et al., 2014).

LTP is mediated by the activation of NMDA and non-NMDA (Kainate and AMPA) receptors (Bliss \& Collingridge, 1993). LTP occurs when both NMDA and AMPA receptors are activated after binding of glutamate released by the presynaptic neuron. Following activation, AMPA receptors allow for influx of sodium ions $\left(\mathrm{Na}^{+}\right)$which depolarize the membrane. The NMDA receptor is permeable to $\mathrm{Ca}^{2+}$; however, it is blocked by a magnesium ion that prevents $\mathrm{Ca}^{2+}$ ions from passing through the receptor. The postsynaptic membrane, thus, must be greatly depolarized by strong signals that are adequate to remove the magnesium ions $\left(\mathrm{Mg}^{++}\right)$from the pore of the NMDA receptor.

When a large amount of glutamate is released from the presynaptic terminal, AMPA receptors open for a longer period thereby allowing a high concentration of $\mathrm{Na}^{+}$to enter the postsynaptic cell. This makes the membrane adequately depolarized to displace the $\mathrm{Mg}^{++}$block from the NMDA receptor and allow for $\mathrm{Ca}^{2+}$ influx. $\mathrm{Ca}^{2+}$ is a second messenger that activates many intracellular cascades. As a result of these intracellular cascades, new AMPArs are synthesized and inserted into the postsynaptic membrane, allowing for stronger response in the future and the expression of LTP (Collingridge \& Bliss, 1987).

In the present study, the AMPA receptor antagonist, NBQX, was used prior to water maze training which presumably blocked LTP thereby preventing learning and memory on the MWM. The morphology of the CA3 neurons was also affected by the AMPAr blockade. These morphological changes are consistent with studies that investigated the effect of LTP on the 
morphology of spines, branching and arborization (Kumar, 2011; Chang \& Greenough, 1984; Petukhov \& Popov, 1986; Mateos, et al., 2007).

One study investigated time-dependent ultrastructural morphological changes on the apical dendrites of CA3 neurons following LTP in vitro (Kumar, 2011). Following LTP induction, two distinct periods were observed in the structural modifications. At first, spine number notably increased and changes in the shape of the active region were seen. These changes were reduced after 2-3 hours while LTP expression remained. The second phase was accented by an increase in the postsynaptic thickness (Kumar, 2011).

A related study evaluated the associations between ultrastructural changes and LTP of giant spinous synapses on the apical dendrites of CA3 neurons (Petukhov \& Popov, 1986). Postsynaptic densities increased in area and length after LTP induction which is consistent with the hypothesis that postsynaptic densities play a role in LTP (Petukhov \& Popov, 1986).

Another study examined in vitro changes in the morphology of hippocampal neurons following LTP, low frequency synaptic activity, or prolonged high frequency activity that did not induce LTP (Chang \& Greenough, 1984). Results revealed that after LTP induction, the quantity of spine synapse increased compared to the slice that received low frequency stimuli. Following a prolonged high frequency synaptic activation that did not lead to an expression of LTP, no change in the number of spines was found. However, when compared to low frequency activation, a transient increase was found in the bouton mitochondrial area and a minor increase in the bouton area (Chang \& Greenough, 1984).

Another study used organotypic slices (living cultures that continue to develop in vitro after their removal from a living organ) of hippocampus to examine the impact of chronic 
AMPA receptor blockade on the synapses between CA3-CA1 pyramidal neurons (Mateos, et al., 2007). Results showed that blocking AMPA receptor, by NBQX, for 14 days evoked a significant decline in dendritic spines on CA1 pyramidal neurons and in synaptic density. Further, chronic AMPA blockade reduced the volume of CA1 pyramidal neurons postsynaptic densities and the quantity of long thin spines. On the contrary, asymmetric presynaptic vesicles on the dendritic shafts of CA3 neurons were increased. This finding indicates compensative alternations in synaptic function and modified bidirectional synaptic plasticity (Mateos, et al., 2007).

The same study also examined synapses in the rest of the hippocampus after NBQX treatment (Mateos, et al., 2007). Results revealed an increase in the hippocampal synaptic number and effectiveness suggesting a counterbalance for the anatomical retractions that occurred in the CA1 pyramidal neurons. Furthermore, these morphological and functional alterations were found to be associated with the modified bidirectional synaptic plasticity in the hippocampus. Thus, it was concluded that normal activation of AMPA receptor is crucial for sustaining structural and functional glutamatergic synapses (Mateos, et al., 2007).

\section{Thorny excrescences function}

Since the first sketch by Ramon y Cajal (1911), the function of thorny excrescences have remained obscure for almost a century. A study by Lee et al. (2013) examined adult and developing hippocampal spines. Results revealed a function of TE in modulating the homeostatic alterations in mature brains but not in developing brains. Homeostatic alteration is a property of the brain in which excitatory networks are internally regulated to assure synaptic efficiency so that excitatory outputs remain stable and relatively constant. Lee et al. study included three 
experiments: two experiments using adult brains and an experiment using developing brains as follows.

In vitro, Tetrodotoxin (TTX), a neurotoxin that blocks sodium channels and inhibits action potentials, was used to investigate the effect of inactivity on the formation of TE and spines in mature hippocampus (Lee et al., 2013). Results revealed that while no changes were detected in global synapses and spines (including distal parts of CA3 neurons), a significant increase was found in the CA3 TE (that were proximal to the cell body) size and synapses (Lee et al., 2013). The significant increase in TE as a reaction to inactivity indicates "TEs are designated homeostatic gain control structures that boost excitatory drive in the mature hippocampus" (Lee et al., 2013).

In a second experiment, Lee and colleagues chronically altered brain activity of adult mice to test if activity alterations have an effect on TE formation in vivo (Lee et al., 2013). Three groups of mice were used and chronically administered picrotoxin (PTX) (a GABAA receptor inhibitor to increase brain activity), diazepam (Dzp) (a GABAA receptor agonist to decrease brain activity) or saline (Lee et al., 2013). Golgi impregnation results revealed a significant reduction in TE formation on CA3 neurons in the PTX-administered group compared to saline group. In contrast, the Dzp-administered group showed a significant increase in the formation of TE on CA3 neurons which is similar to the effect of TTX in vitro in adult brains. These results indicate bidirectional modifications in the TE formation as responses to activity alternations in the brain (Lee et al., 2013).

During development, a distinct mechanism of adaptation, which does not occur in adulthood, regulates the excitatory neural activities in the brain called "synaptic scaling" 
(Turrigiano \& Nelson, 2000). Synaptic scaling refers to the contingent synaptic weights that are conserved during development by homogeneous and general changes in excitatory synapses in the entire brain (Turrigiano \& Nelson, 2000). Thus, Lee et al. used developing brains to test the effect of inactivity on hippocampal spines and TE formation. In vitro, developing hippocampal slices were chronically treated with TTX (Lee et al., 2013). Contrary to what was observed in mature brains mentioned above, results revealed a significant increase in global spines and synapses (including spines and synapses on CA3 proximal and distal dendrites) indicating synaptic scaling. This finding shows that TE role during development does not involve homeostatic alternations (Lee et al., 2013).

The study by Lee and colleagues revealed that, in adulthood, TE allow for homeostatic modifications without disturbing storage of synaptic information in other parts of the hippocampal network (Lee et al., 2013). In the present thesis, TE in the developing groups in Experiment 1 showed a significant increase with age suggesting biological and morphological changes that are crucial for adaptation and regulation of excitatory activities perhaps into adulthood. The adaptive function of TE is also suggested in the current study to have a role in spatial learning and memory. TE in Experiment 2 decreased in number after AMPA receptor antagonist injections while spines were increased. This suggest a synaptic scaling which is similar to the effect of TTX on developing brains found in Lee et al. (2013) study described above. 


\section{Limitations}

There were limitations regarding the use of Golgi-cox impregnation method. First, the reason why only selected cells respond to the Golgi impregnation is unknown. This makes it possible that the reconstructed neurons in the study might be not necessarily a good representation of the overall brain integrity. During the search for a proper neuron in the current study, it was noticed that some CA3 neurons were larger or smaller in the same slice. Therefore, a middle sized neuron was selected for reconstruction avoiding the extremely small or large sized ones.

Another limitation is that impregnated neurons sometimes overlapped making it difficult to find a neuron that was not interfered with by nearby dendrites. Thus, neurons were carefully selected although some of the reconstructed neurons in this study were overlapped by neighboring branches.

CA3 pyramidal neurons are localized at the folded part of the hippocampus, making them placed at the vertical axis as opposed to horizontal. This makes it difficult to find a whole neuron that is not cut while obtaining brain sections. In some cases, when a neuron was complete, it was difficult to keep in focus. As a result, finding a neuron, then reconstructing it in the CA3 hippocampal area was time consuming. Therefore, in this study, fewer neurons were used for reconstructions (4 neurons per group in both experiments). A larger number of reconstructed neurons may lead to more significant statistical outcomes for the study.

Quantification of thorny excrescences on the cell bodies of CA3 neurons may not be precise as there is no known way to focus through the cell body and see TE on the other half of it. It is possible that there are some TE that were not counted. Moreover, this study provided a 
quantification of TE regardless of their size, length or thickness. A TE could be a complex shaped spine-like (larger than a spine), or it could be a large cylinder (similar to a branch but smaller) with clusters of spines on it. There is no known method or technology, to the time of writing this study, which allows for exact measurement of the size or complexity of a TE.

\section{Conclusions}

The results from this study demonstrated morphological changes in the structure of CA3 pyramidal neurons during the developmental period $\mathrm{p} 18$ to $\mathrm{p} 24$. These changes are suggested to have a role in the emergence of spatial abilities at p24. Moreover, an increase is the number of thorny excrescences was found indicating TE function in begetting spatial information through the DG-CA3 circuitry. Experiment 2 showed changes in the CA3 morphology after administration of NBQX, an AMPA receptor antagonist, and training on the MWM. In addition, TE number decreased after injections. The structural alterations following NBQX injections were correlated with impairments in the performance on the MWM. These results suggest a function of AMPA receptors in the formation of spatial memories along with thorny excrescences in CA3 pyramidal neurons. 


\section{References}

Acsa'dy, L., Kamondi, A., Si'k, A., Freund, T., \& Buzsa'ki G. (1998). GABAergic cells are the major postsynaptic targets of mossy fibers in the rat hippocampus. The Journal of Neuroscience, 18, 3386-3403.

Agnati, L. F., Genedani, S., Leo, G., Rivera, A., Guidolin, D., \& Fuxe, K. (2007). One century of progress in neuroscience founded on Golgi and Cajal's outstanding experimental and theoretical contributions. Brain Research Reviews, 55(1), 176-189.

Ahlander, M., Misane, I., Schott, P. A., \& Ogren, S. O. (1999). A behavioral analysis of the spatial learning deficit induced by the NMDA receptor antagonist MK-801 (dizocilpine) in the rat. Neuropsychopharmacology, 21(3), 414-426.

Akers, K. G., Candelaria-Cook, F. T., Rice, J. P., Johnson, T. E., \& Hamilton, D. A. (2009). Delayed Development of Place Navigation Compared to Directional Responding in Young Rats. Behavioral Neuroscience, 123(2), 267-275. doi: 10.1037/a0014594

Akers, K. G., Candelaria-Cook, F. T., Rice, J. P., Johnson, T. E., \& Hamilton, D. A. (2011). Cued Platform Training Reveals Early Development of Directional Responding Among Preweanling Rats in the Morris Water Task. Developmental Psychobiology, 53(1), 1-12. doi: $10.1002 / \mathrm{dev} .20480$

Akers, K. G., Candelaria, F. T., \& Hamilton, D. A. (2007). Preweanling rats solve the Morris water task via directional navigation. Behavioral Neuroscience, 121(6), 1426-1430. doi: $10.1037 / 0735-7044.121 .6 .1426$ 
Akers, K. G., \& Hamilton, D. A. (2007). Comparison of developmental trajectories for place and cued navigation in the Morris water task. Developmental Psychobiology, 49, 553-564.

Altman, J. (1969). Autoradiographic and histological studies of postnatal neurogenesis. IV. Cell proliferation and migration in the anterior forebrain, with special reference to persisting neurogenesis in the olfactory bulb. Journal of Comparative Neurology, 137(4), 433-457.

Amaral, D.G. (1978). A Golgi study of cell types in the hilar region of the hippocampus in the rat. The Journal of Comparative Neurology, 182, 851-914.

Amaral, D. G., \& Dent, J. A. (1981). Development of the mossy fibers of the dentate gyrus: I. A light and electron microscopic study of the mossy fibers and their expansions. The Journal of Comparative Neurology, 195, 51-86.

Amaral, D., \& Witter, M. (1989). The three-dimensional organization of the hippocampal formation: a review of anatomical data. Neuroscience, 31(3), 571-591.

Andersen, P., Bliss, T., \& Skrede, K. K. (1971). Lamellar organization of hippocampal excitatory pathways. Experimental Brain Research, 13(2), 222-238.

Andrews, T. J., Thrasivoulou, C., Nesbit, W., \& Cowen, T. (1996). Target-specific differences in the dendritic morphology and neuropeptide content of neurons in the rat SCG during development and aging. Journal of Comparative Neurology, 368(1), 33-44.

Bannerman, D., Yee, B., Good, M., Heupel, M., Iversen, S., \& Rawlins, J. (1999). Double dissociation of function within the hippocampus: a comparison of dorsal, ventral, and complete hippocampal cytotoxic lesions. Behavioral neuroscience, 113(6), 1170. 
Barnes, C. (1988). Spatial learning and memory processes: the search for their neurobiological mechanisms in the rat. Trends in neurosciences, 11(4), 163-169.

Bayer, S. A. (1979). The development of the septal region in the rat. I. Neurogenesis examined with 3H-thymidine autoradiography. Journal of Comparative Neurology, 183(1), 89-106.

Bayer, S. A. (1980). Development of the hippocampal region in the rat II. Morphogenesis during embryonic and early postnatal life. Journal of Comparative Neurology, 190(1), 115-134.

Bell, H. C., Pellis, S. M., \& Kolb, B. (2010). Juvenile peer play experience and the development of the orbitofrontal and medial prefrontal cortices. Behavioural Brain Research, 207(1), 7 13.

Blackstad, T.W. (1956). Commissural connections of the hippocampal region in the rat, with special reference to their mode of termination. Journal of Comparative Neurology, 105, 417-537.

Blackstad, T. W., \& Kjaerheim, A. (1961). Special axo-dendritic synapses in the hippocampal cortex: electron and light microscopic studies on the layer of mossy fibers. The Journal of Comparative Neurology, 117, 133-159.

Blair, M. G., Nguyen, N. N., Albani, S. H., L'Etoile M, M., Andrawis, M. M., Owen, L. M., Dumas, T. C. (2013). Developmental Changes in Structural and Functional Properties of Hippocampal AMPARs Parallels the Emergence of Deliberative Spatial Navigation in Juvenile Rats. J Neurosci, 33(30), 12218-12228. doi: 10.1523/JNEUROSCI.4827-12.2013

Bliss, T. V., \& Collingridge, G. L. (1993). A synaptic model of memory: long-term potentiation in the hippocampus. Nature, 361(6407), 31-39. 
Brown, R. W., \& Whishaw, I. Q. (2000). Similarities in the development of place and cue navigation by rats in a swimming pool. Developmental Psychobiology, 37(4), 238-245.

Carman, H. M., \& Mactutus, C. F. (2001). Ontogeny of spatial navigation in rats: A role for response requirements? Behavioral Neuroscience, 115, $870-879$.

Cassel, J., Cassel, S., Galani, R., Kelche, C., Will, B., \& Jarrard, L. (1998). Fimbria-fornix vs selective hippocampal lesions in rats: effects on locomotor activity and spatial learning and memory. Neurobiology of learning and memory, 69(1), 22-45.

Chang, F. F., \& Greenough, W. T. (1984). Transient and enduring morphological correlates of synaptic activity and efficacy change in the rat hippocampal slice. Brain research, 309(1), 35-46.

Cherubini, E., \& Miles, R. (2015). The CA3 region of the hippocampus: how is it? What is it for? How does it do it? Frontiers in Cellular Neuroscience, 9, 19. doi:10.3389/fncel.2015.00019

Chicurel, M. E., \& Harris, K. M. (1992). Three-dimensional analysis of the structure and composition of CA3 branched dendritic spines and their synaptic relationships with mossy fiber boutons in the rat hippocampus. Journal of Comparative Neurology, 325, 169-182.

Chechik, G., Meilijson, I., \& Ruppin, E. (1998). Synaptic pruning in development: a computational account. Neural computation, 10(7), 1759-1777.

Cintra, L., Díaz-Cintra, S., Galván, A., Kemper, T., \& Kemper, P. J. (1990). Effects of protein undernutrition on the dentate gyrus in rats of three age groups. Brain Research, 532(1-2) (27), 1-7. 
Collingridge, G.L., \& Bliss, T.V.P. (1987). NMDA receptors - their role in long-term potentiation. Trends in Neuroscience, 7, 288-293.

Cotman, C.W., Monaghan, D.T. \& Ganong, A.H. (1988). Excitatory amino acid neurotransmission: NMDA receptors and Hebb-type synaptic plasticity. Annual Review Neuroscience 11, 61-80.

Czéh, B., Stuchlik, A., Wesierska, M., Cimadevilla, J. M., Pokorný, J., Seress, L., et al. (2001). Effect of neonatal dentate gyrus lesion on allothetic and idiothetic navigation in rats. Neurobiology of learning and memory, 75(2), 190-213.

Danscher, G., \& Zimmer, J. (1978). An improved Timm sulphide silver method for light and electron microscopic localization of heavy metals in biological tissues. Histochemistry and Cell Biology, 55(1), 27-40.

Davies, J., Evans, R., Jones, A., Smith, D., \& Watkins, J. (1982). Differential activation and blockade of excitatory amino acid receptors in the mammalian and amphibian central nervous systems. Comparative Biochemistry and Physiology Part C: Comparative Pharmacology, 72(2), 211-224.

De Carlos, J. A., \& Borrell, J. (2007). A historical reflection of the contributions of Cajal and Golgi to the foundations of neuroscience. Brain Research Reviews, 55, 8-16.

D'Hooge, R., \& De Deyn, P. P. (2001). Applications of the Morris water maze in the study of learning and memory. Brain research reviews, 36(1), 60-90. 
Díaz-Cintra, S., García-Ruiz, M., Corkidi, G., \& Cintra, L. (1994). Effects of prenatal malnutrition and postnatal nutritional rehabilitation on ca3 hippocampal pyramidal cells in rats of four ages. Brain Research, 662(1-2), 117-126.

Dickstein, D. L., Kabaso, D., Rocher, A. B., Luebke, J. I., Wearne, S. L., \& Hof, P. R. (2007). Changes in the structural complexity of the aged brain. Aging Cell, 6(3), 275-284. doi:10.1111/j.1474-9726.2007.00289.x

Dong, H., O'Brien, R. J., Fung, E. T., Lanahan, A. A., Worley, P. F., \& Huganir, R. L. (1997). GRIP: a synaptic PDZ domain-containing protein that interacts with AMPA receptors. $386(6622), 279-284$.

Douglas, R. J., Peterson, J. J., \& Douglas, D. P. (1973). The ontogeny of a hippocampusdependent response in two rodent species. Behav Biol, 8(1), 27-37.

Dumas, T. C. (2004). Early eyelid opening enhances spontaneous alternation and accelerates the development of perforant path synaptic strength in the hippocampus of juvenile rats. Developmental Psychobiology, 45(1), 1-9. doi: 10.1002/dev.20011

Eiland, L., Ramroop, J., Hill, M. N., Manley, J., \& McEwen, B. C. (2012). Chronic juvenile stress produces corticolimbic dendritic architectural remodeling and modulates emotional behavior in male and female rats. Psychoneuroendocrinology, 37(1), 39-47.

El Iskandrani, K., Oosterhof, C., El Mansari, M., \& Blier, P. (2015). Impact of subanesthetic doses of ketamine on AMPA-mediated responses in rats: An in vivo electrophysiological study on monoaminergic and glutamatergic neurons. Journal of Psychopharmacology. 
Fitch JM, Juraska JM \&Washington LW. (1989). The dendritic morphology of pyramidal neurons in the rat hippocampal CA3 area. I. Cell types. Brain Research. 479, 105-114.

Floresco, S. (2014, April 16). Spatial Learning in Animals. Retrieved from http://link.springer.com/referenceworkentry/10.1007/978-3-642-27772-6 354-2?no$\underline{\text { access }=\text { true }}$

Florian, C., \& Roullet, P. (2004). Hippocampal CA3-region is crucial for acquisition and memory consolidation in Morris water maze task in mice. Behavioural brain research, 154(2), 365-374.

Frederickson, C. J., Klitenick, M. A., Manton, W. I., \& Kirkpatrick, J. B. (1983). Cytoarchitectonic distribution of zinc in the hippocampus of man and the rat. Brain research, 273(2), 335-339.

Gage, F. H., \& Bjorklund, A. (1986). Cholinergic septal grafts into the hippocampal formation improve spatial learning and memory in aged rats by an atropine-sensitive mechanism. The Journal of neuroscience, 6(10), 2837-2847.

González-Burgos, I., Alejandre-Gomez, M., Olvera-Cortes, M. E., Perez-Vega, M. I., Evans, S., \& Perez-Vega, A. (2006). Prenatal-through-postnatal exposure to moderate levels of ethanol leads to damage on the hippocampal CA1 field of juvenile rats: a stereology and Golgi study. Neuroscience Research, 56(4), 400-408.

Gonzales, R. B., DeLeon Galvan, C. J., Rangel, Y. M., \& Claiborne, B. J. (2001). Distribution of thorny excrescences on CA3 pyramidal neurons in the rat hippocampus. Journal of Comparative Neurology, 430(3), 357-368. 
Green, R. J., \& Stanton, M. E. (1989). Differential ontogeny of working memory and reference memory in the rat. [Research Support, Non-U.S. Gov't Research Support, U.S. Gov't, P.H.S.]. Behav Neurosci, 103(1), 98-105.

Hampson, D., Huang, X., Oberdorfer, M., Goh, J., Auyeung, A., \& Wenthold, R. (1992). Localization of AMPA receptors in the hippocampus and cerebellum of the rat using an anti-receptor monoclonal antibody. Neuroscience, 50(1), 11-22.

Harris, K., Jensen F., Tsao, B. (1992). Three-dimensional structure of dendritic spines and synapses in rat hippocampus (CA1) at postnatal day 15 and adult ages: implications for the maturation of synaptic physiology and long-term potentiation. Journal of Neuroscience, $12,2685-2705$.

Holahan, M. R., Rekart, J. L., Sandoval, J., \& Routtenberg, A. (2006). Spatial learning induces presynaptic structural remodeling in the hippocampal mossy fiber system of two rat strains. Hippocampus, 16(6), 560-570.

Holahan, M. R., Honegger, K. S., \& Routtenberg, A. (2007). Expansion and retraction of hippocampal mossy fibers during postweaning development: Strain-specific effects of NMDA receptor blockade. Hippocampus, 17(1), 58-67.

Holahan, M. R., \& Smith, C. A. (2015). Phthalates and neurotoxic effects on hippocampal network plasticity. Neurotoxicology, 48, 21-34.

Holahan, M. R., \& Routtenberg, A. (2011). Lidocaine injections targeting CA3 hippocampus impair long-term spatial memory and prevent learning-induced mossy fiber remodeling. Hippocampus, 21(5), 532-540. 
Hollmann, M., \& Heinemann, S. (1994). Cloned glutamate receptors. Annual review of neuroscience, 17(1), 31-108.

Holtmaat, A., \& Svoboda, K. (2009). Experience-dependent structural synaptic plasticity in the mammalian brain. Nature Reviews Neuroscience, 10(9), 647-658.

Honoré, T., Lauridsen, J., \& Krogsgaard-Larsen, P. (1982). The binding of [3H] AMPA, a structural analogue of glutamic acid, to rat brain membranes. Journal of neurochemistry, $38(1), 173-178$.

Hunsaker, M. R., Lee, B., \& Kesner, R. P. (2008). Evaluating the temporal context of episodic memory: the role of CA3 and CA1. Behavioural brain research, 188(2), 310-315.

Hunsaker, M. R., Thorup, J. A., Welch, T., \& Kesner, R. P. (2006). The role of CA3 and CA1 in the acquisition of an object-trace-place paired-associate task. Behavioral neuroscience, 120(6), 1252.

Jablonski, S. A., Schiffino, F. L., \& Stanton, M. E. (2012). Role of age, post-training consolidation, and conjunctive associations in the ontogeny of the context preexposure facilitation effect. Developmental Psychobiology, 54(7), 714-722. doi: 10.1002/dev.20621

Jarrard, L. E. (1993). On the role of the hippocampus in learning and memory in the rat. Behavioral and neural biology, 60(1), 9-26.

John O'Keefe \& Lynn Nadel (1978) The Hippocampus as a Cognitive Map, Oxford University Press. 
Keeley, R.J., Wartman, B.C., Hausler, A.N., \& Holahan, M.R. (2010). Effect of juvenile pretraining on adolescent structural hippocampal attributes as a substrate for enhanced spatial performance. Learning and Memory, 17, 344-354

Kesner, R. P., Hunsaker, M. R., \& Warthen, M. W. (2008). The CA3 subregion of the hippocampus is critical for episodic memory processing by means of relational encoding in rats. Behavioral neuroscience, 122(6), 1217.

Kumar, A. (2011). Long-Term Potentiation at CA3-CA1 Hippocampal Synapses with Special Emphasis on Aging, Disease, and Stress. Frontiers in Aging Neuroscience, 3, 7. doi:10.3389/fnagi.2011.00007

Langston, R. F., Ainge, J. A., Couey, J. J., Canto, C. B., Bjerknes, T. L., Witter, M. P., . . Moser, M. B. (2010). Development of the spatial representation system in the rat. [Research Support, Non-U.S. Gov't]. Science, 328(5985), 1576-1580. doi: 10.1126/science. 1188210

Lawrence, R. C., Otero, N. C., \& Kelly, S. J. (2012). Selective effects of perinatal ethanol exposure in medial prefrontal cortex and nucleus accumbens. Neurotoxicology and Teratology, 34(1), 128-135.

Lee, K. J., Queenan, B. N., Rozeboom, A. M., Bellmore, R., Lim, S. T., Vicini, S., et al. (2013). Mossy fiber-CA3 synapses mediate homeostatic plasticity in mature hippocampal neurons. Neuron, 77(1), 99-114.

Luebke, J. I., \& Rosene, D. L. (2003). Aging alters dendritic morphology, input resistance, and inhibitory signaling in dentate granule cells of the rhesus monkey. Journal of Comparative Neurology, 460(4), 573-584. 
Malenka, R.C. (1994). Synaptic plasticity in the hippocampus: LTP and LTD. Cell. 78, 535-538.

Mao, L., Xue, B., Jin, D., \& Wang, J. Q. (2015). Dynamic increases in AMPA receptor phosphorylation in the rat hippocampus in response to amphetamine. Journal of neurochemistry.

Markham, J., McKian, K., Stroup, T., \& Juraska, J. (2005). Sexually dimorphic aging of dendritic morphology in CA1 of hippocampus. Hippocampus, 15(1), 97-103.

Mateos, J. M., Lüthi, A., Savic, N., Stierli, B., Streit, P., Gähwiler, B. H., \& McKinney, R. A. (2007). Synaptic modifications at the CA3-CA1 synapse after chronic AMPA receptor blockade in rat hippocampal slices. The Journal of Physiology, 581(Pt 1), 129-138. doi:10.1113/jphysiol.2006.1205504

McAllister, A. K. (2000). Cellular and molecular mechanisms of dendrite growth. Cerebral Cortex, 10(10), 963-973.

McDonald, R. J., Hong, N. S., \& Devan, B. D. (2004). The challenges of understanding mammalian cognition and memory-based behaviours: an interactive learning and memory systems approach. Neuroscience \& Biobehavioral Reviews, 28(7), 719-745.

McGonigal, R., Tabatadze, N., \& Routtenberg, A. (2012). Selective presynaptic terminal remodeling induced by spatial, but not cued, learning: A quantitative confocal study. Hippocampus, 22(6), 1242-1255.

Monroy, E., Hernández-Torres, E., \& Flores, G. (2010). Maternal separation disrupts dendritic morphology of neurons in prefrontal cortex, hippocampus, and nucleus accumbens in male rat offspring. Journal of Chemical Neuroanatomy, 40(2), 93-101. 
Morris, R. G. (1981). Spatial localization does not require the presence of local cues. Learning and motivation, 12(2), 239-260.

Morris, R. (1984). Developments of a water-maze procedure for studying spatial learning in the rat. Journal of neuroscience methods, 11(1), 47-60.

Morris, R., Schenk, F., Tweedie, F., \& Jarrard, L. (1990). Ibotenate lesions of hippocampus and/or subiculum: dissociating components of allocentric spatial learning. European Journal of Neuroscience, 2(12), 1016-1028.

Moser, M., Trommald, M., \& Andersen, P. (1994). An increase in dendritic spine density on hippocampal CA1 pyramidal cells following spatial learning in adult rats suggests the formation of new synapses. Proceedings of the National Academy of Sciences, 91(26), 12673-12675.

Moser, M., Trommald, M., Egeland, T., \& Andersen, P. (1997). Spatial training in a complex environment and isolation alter the spine distribution differently in rat CA1 pyramidal cells. Journal of Comparative Neurology, 380(3), 373-381.

Nabavi, S., Fox, R., Proulx, C. D., Lin, J. Y., Tsien, R. Y., \& Malinow, R. (2014). Engineering a memory with LTD and LTP. Nature.

Nakanishi, S., \& Masu, M. (1994). Molecular diversity and functions of glutamate receptors. Annual Review of Biophysics and Biomolecular Structure. 23, 319-348.

Narayanan, R., Narayan, A., \& Chattarji, S. (2005). A probabilistic framework for regionspecific remodeling of dendrites in three-dimensional neuronal reconstructions. Neural computation, 17(1), 75-96. 
Norrholm, S. D., \& Ouimet, C. C. (2000). Chronic fluoxetine administration to juvenile rats prevents age-associated dendritic spine proliferation in hippocampus. Brain Research, 883(2), 205-215.

Norton, S., \& Culver, B. (1977). A Golgi analysis of caudate neurons in rats exposed to carbon monoxide. Brain Research, 132(3), 455-465.

Olton, D. S., \& Paras, B. C. (1979). Spatial memory and hippocampal function. Neuropsychologia, 17(6), 669-682.

Pandey, S. P., Rai, R., Gaur, P., \& Prasad, S. (2015). Development-and age-related alterations in the expression of AMPA receptor subunit GluR2 and its trafficking proteins in the hippocampus of male mouse brain. Biogerontology, 1-12.

Petukhov, V., \& Popov, V. (1986). Quantitative analysis of ultrastructural changes in synapses of the rat hippocampal field CA3 in vitro in different functional states. Neuroscience, 18(4), $823-835$.

Poucet, B., \& Benhamou, S. (1997). The neuropsychology of spatial cognition in the rat. Critical Reviews $^{\mathrm{TM}}$ in Neurobiology, 11(2-3).

Radecki, D. T., Brown, L. M., Martinez, J., \& Teyler, T. J. (2005). BDNF protects against stressinduced impairments in spatial learning and memory and LTP. Hippocampus, 15(2), 246253.

Raisman, G., Cowan, W.M. \& Powell, T.P.S. (1965). The extrinsic afferent, commissural and association fibres of the hippocampus. Brain, 88, 963-996. 
Rakic, P., Bourgeois, J., \& Goldman-Rakic, P. S. (1994). Synaptic development of the cerebral cortex: implications for learning, memory, and mental. The Self-Organizing Brain: From Growth Cones to Functional Networks, 102, 227.

Ramírez-Amaya, V., Balderas, I., Sandoval, J., Escobar, M. L., \& Bermúdez-Rattoni, F. (2001). Spatial long-term memory is related to mossy fiber synaptogenesis. The Journal of Neuroscience, 21(18), 7340-7348.

Ramon y Cajal, S. (1911). Histologie du Syste 'me Nerveux de l'homme et des Verte' bre' s (Paris: Maloine).

Rekart, J. L., Sandoval, C. J., Bermudez-Rattoni, F., \& Routtenberg, A. (2007). Remodeling of hippocampal mossy fibers is selectively induced seven days after the acquisition of a spatial but not a cued reference memory task. Learning \& Memory, 14(6), 416-421.

Richards, S., Mychasiuk, R., Kolb, B., \& Gibb, R. (2012). Tactile stimulation during development alters behaviour and neuroanatomical organization of normal rats. Behavioural Brain Research, 231(1), 86-91.

Rossier, J., \& Schenk, F. (2003). Olfactory and/or visual cues for spatial navigation through ontogeny: olfactory cues enable the use of visual cues. Behavioral neuroscience, 117(3), 412.

Rudy, J. W., \& Paylor, R. (1987). Development of interocular equivalence of place learning in the rat requires convergence sites established prior to training. Behavioral neuroscience, 101(5), 732. 
Rudy, J. W. (1993). Contextual conditioning and auditory cue conditioning dissociate during development. Behav Neurosci, 107(5), 887-891.

Rudy, J. W., Stadler-Morris, S., \& Albert, P. (1987). Ontogeny of spatial navigation behaviors in the rat: dissociation of" proximal"-and" distal"-cue-based behaviors. Behavioral neuroscience, 101(1), 62.

Rudy, J. W., \& Morledge, P. (1994). Ontogeny of contextual fear conditioning in rats: implications for consolidation, infantile amnesia, and hippocampal system function. Behavioral Neuroscience, 108(2), 227-234.

Salt, T.E., \& Eaton, S.A. (1996). Functions of ionotropic and metabotropic glutamate receptors in sensory transmission in the mammalian thalamus. Progress in Neurobiology, 48, 55-72.

Scharfman, H. E. (2007). The CA3 "backprojection" to the dentate gyrus. Progress in brain research, 163, 627-637.

Sharma, S., Rakoczy, S., \& Brown-Borg, H. (2010). Assessment of spatial memory in mice. Life sciences, 87(17), 521-536.

Shi, S., Hayashi, Y., Petralia, R. S., Zaman, S. H., Wenthold, R. J., Svoboda, K., et al. (1999). Rapid spine delivery and redistribution of AMPA receptors after synaptic NMDA receptor activation. Science, 284(5421), 1811-1816.

Silva-Gomez, A. B., Rojas, D., Juarez, I., \& Flores, G. (2003). Decreased dendritic spine density on prefrontal cortical and hippocampal pyramidal neurons in postweaning social isolation rats. Brain Research, 983, 128-136. 
Sloviter, R. S., \& Lømo, T. (2012). Updating the lamellar hypothesis of hippocampal organization. Frontiers in neural circuits, 6.

Smith, C. A., \& Holahan, M. R. (2014). Reduced Hippocampal Dendritic Spine Density and BDNF Expression following Acute Postnatal Exposure to Di (2-Ethylhexyl) Phthalate in Male Long Evans Rats. PloS one, 9(10), e109522.

Smith, C. A., Macdonald, A., \& Holahan, M. R. (2011). Acute postnatal exposure to di(2ethylhexyl) phthalate adversely impacts hippocampal development in the male rat. [Research Support, Non-U.S. Gov't]. Neuroscience, 193, 100-108. doi: 10.1016/j.neuroscience.2011.06.082

Stewart, M. G., Davies, H. A., Sandi, C., Kraev, I. V., Rogachevsky, V. V., Peddie, C. J., et al. (2005). Stress suppresses and learning induces plasticity in CA3 of rat hippocampus: a three-dimensional ultrastructural study of thorny excrescences and their postsynaptic densities. Neuroscience, 131(1), 43-54.

Stoltenburg-Didinger, G., \& Spohr, H. L. (1983). Fetal alcohol syndrome and mental retardation: Spine distribution of pyramidal cells in prenatal alcohol-exposed rat cerebral cortex; a Golgi study. Developmental Brain Research, 11(1), 119-123.

Stirling, R. V., \& Bliss, T. V. P. (1978). Hippocampal mossy fiber development at the ultrastructural level. Progress in Brain Research, 48, 191-198.

Teyler, T. J., \& DiScenna, P. (1984). The topological anatomy of the hippocampus: A clue to its function. Brain Research Bulletin, 12, 711-719. 
"The Nobel Prize in Physiology or Medicine 1906". Nobelprize.org. Nobel Media AB 2013. Web. 22 Aug 2013.

Tonkiss, J., Shultz, P., \& Galler, J. R. (1992). Long-Evans and spargue-dawley rats differ in their spatial navigation performance during ontogeny and at maturaty. Developmental Psychobiology, 25(8), 567-579. doi: 10.1002/dev.420250804

Turrigiano, G. G., \& Nelson, S. B. (2000). Hebb and homeostasis in neuronal plasticity. Current opinion in neurobiology, 10(3), 358-364.

Vida, I., \& Frotscher, M. (2000). A hippocampal interneuron associated with the mossy fiber system. Proceedings of the National Academy of Sciences, 97(3), 1275-1280.

Vorhees, C. V., \& Williams, M. T. (2006). Morris water maze: procedures for assessing spatial and related forms of learning and memory. Nature protocols, l(2), 848-858.

Wartman, B. C., Gervais, N. J., Smith, C. A., Comba, R., Mumby, D. G., \& Holahan, M. R. (2012). Enhanced adolescent learning and hippocampal axonal projections following preadolescent spatial exposure to a water or dry maze. Brain Research, 475, 12.

Wills, T. J., Cacucci, F., Burgess, N., \& O'Keefe, J. (2010). Development of the Hippocampal Cognitive Map in Preweanling Rats. Science, 328(5985), 1573-1576. doi: $10.1126 /$ science. 1188224

Wisden, W., \& Seeburg, P.H. (1993). Mammalian ionotropic glutamate receptors. Current Opinion in Neurobiology 3, 291-298.

Wu, G. Y., Zou, D. J., Rajan, I., \& Cline, H. (1999). Dendritic dynamics in vivo change during neuronal maturation. The Journal of neuroscience, 19(11), 4472-4483. 
Xie, M., Yan, J., He, C., Yang, L., Tan, G., Li, C., et al. (2015). Short-term sleep deprivation impairs spatial working memory and modulates expression levels of ionotropic glutamate receptor subunits in hippocampus. Behavioural brain research. 